\title{
WestVirginiaUniversity
}

THE RESEARCH REPOSITORY @ WVU

Graduate Theses, Dissertations, and Problem Reports

2013

\section{Degradation studies of flexible optoelectronic device electrodes}

Theodros S. Bejitual

West Virginia University

Follow this and additional works at: https://researchrepository.wvu.edu/etd

\section{Recommended Citation}

Bejitual, Theodros S., "Degradation studies of flexible optoelectronic device electrodes" (2013). Graduate Theses, Dissertations, and Problem Reports. 4951.

https://researchrepository.wvu.edu/etd/4951

This Dissertation is protected by copyright and/or related rights. It has been brought to you by the The Research Repository @ WVU with permission from the rights-holder(s). You are free to use this Dissertation in any way that is permitted by the copyright and related rights legislation that applies to your use. For other uses you must obtain permission from the rights-holder(s) directly, unless additional rights are indicated by a Creative Commons license in the record and/ or on the work itself. This Dissertation has been accepted for inclusion in WVU Graduate Theses, Dissertations, and Problem Reports collection by an authorized administrator of The Research Repository @ WVU.

For more information, please contact researchrepository@mail.wvu.edu. 


\title{
DEGRADATION STUDIES OF FLEXIBLE OPTOELECTRONIC DEVICE ELECTRODES
}

\author{
Theodros S. Bejitual \\ Dissertation submitted to the \\ Benjamin M. Statler College of Engineering and Mineral Resources \\ at West Virginia University \\ in partial fulfillment of the requirements \\ for the degree of
}

Doctor of Philosophy

in

Mechanical Engineering

Darran Cairns, Ph.D., Chair

Konstantinos Sierros, Ph.D.

Xueyan Song, Ph.D.

Xingbo Liu, Ph.D.

Charter Stinespring, Ph.D.

Department of Mechanical and Aerospace Engineering

Morgantown, West Virginia

2013

Keywords: Fatigue-corrosion; ITO; CNT; PET; Optoelectronic

Copyright 2013 Theodros S. Bejitual 


\section{ABSTRACT \\ DEGRADATION STUDIES OF FLEXIBLE OPTOELECTRONIC DEVICE ELECTRODES Theodros S. Bejitual}

Flexible transparent electrodes offer significant advantages, such as low cost, large area, light weight, conformability, robustness, and ease of roll-to-roll manufacturing and processing. They are routinely used as anodes in organic light emitting diodes, liquid crystal displays, touch panels, solar cells, solid state lightings, energy harvesting, and biomedical applications to name a few examples. However, the electromechanical and corrosion issues involved when the device is stressed and/or in contact with acid containing components both during manufacturing and/or in service conditions have to be investigated in order to improve, and predict reliability.

The primary objective of this research is to investigate the degradation behavior of two types of flexible transparent conducting layers, indium tin oxide (ITO) and carbon nanotubes (CNT) on polymer substrates, under electromechanical and corrosion conditions. Changes in electrical resistance and morphological features of these thin film electrodes are investigated using experimental methods such as corrosion, bending, fatigue, bending-corrosion, fatigue-corrosion, and tribo-corrosion. Such methods attempt to simulate induced stresses during manufacturing and/or in-service conditions. Studies on both patterned and non-patterned surfaces are performed.

Furthermore, finite element modeling is used to simulate the stress/strain distribution of the electrodes under various deformation modes. The effects and synergies of corrosion, applied strain, film thickness, and number of bending cycles on the electrical and structural integrity of the electrodes are investigated using design of experiment methods.

During this project it was found that CNT-based electrodes outperform their ITO counterparts under fatigue in corrosive environments. However, for most high current electronic devices ITO still needs to be utilized. During combined fatigue corrosion experiments of ITO-coated polymer electrodes externally applied strain was found to be the most critical factor for degradation. Experimental analysis and modeling of thin film electrodes for flexible optoelectronics will aid towards the design of more reliable devices in the future. 


\section{ACKNOWLEDGMENTS}

I am sincerely grateful to my advisor, Dr. Darran Cairns, for his guidance, research support, and encouragement during this research project. I would like to thank my committee members, Dr. Konstantinos Sierros, Dr. Xueyan Song, Dr. Xingbo Liu, and Dr. Charter Stinespring for their devoted support for my Ph.D. study. In particular, I would like to express my sincere gratitude to Dr. Sierros for his mentorship, motivation, support, inspiring discussions, and invaluable comments throughout my study.

I would also like to thank all my colleagues in the flexible device laboratory at WVU, especially Aaron Kessman and Nicholas Morris for all their support and comments.

I am thankful also to Clifford Judy for helping me during machining of various custom-built apparatuses.

I am grateful to CPFilms Inc. and Grote Industries Inc. for the funding and material support during my research.

Finally, I would like to thank all my friends and family for their continuous support and encouragement during my studies. 


\section{TABLE OF CONTENTS}

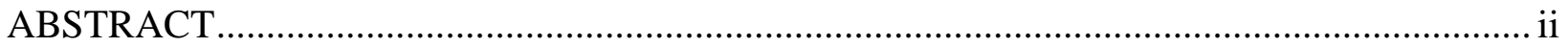

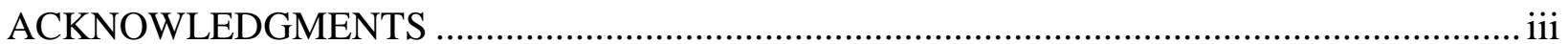

LIST OF TABLES ...................................................................................................................

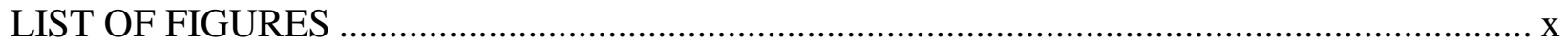

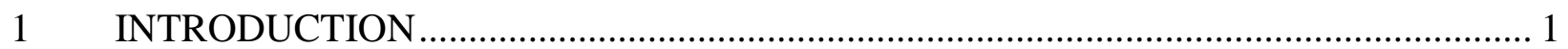

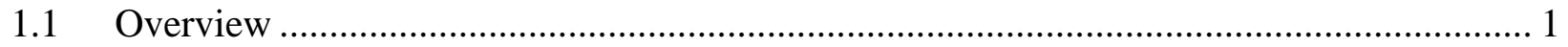

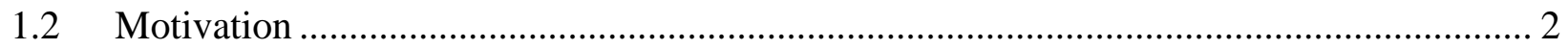

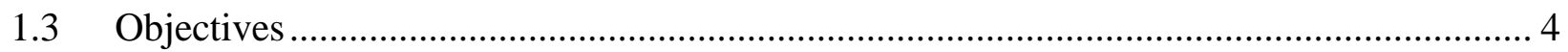

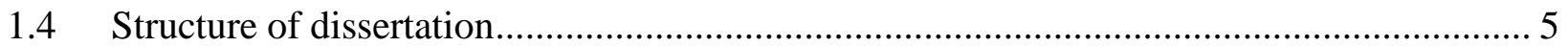

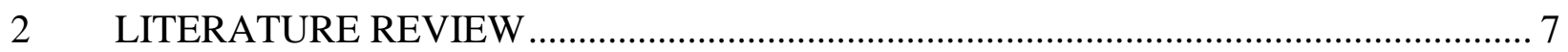

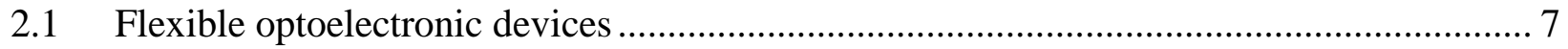

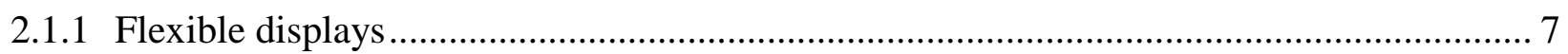

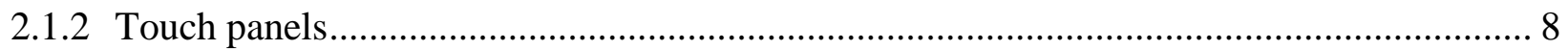

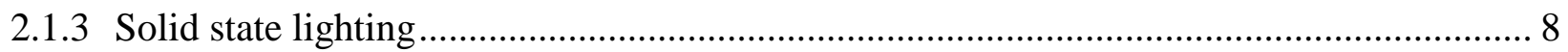

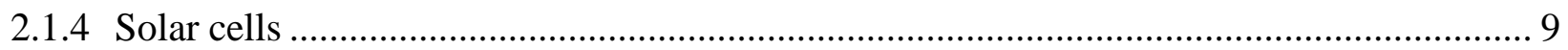

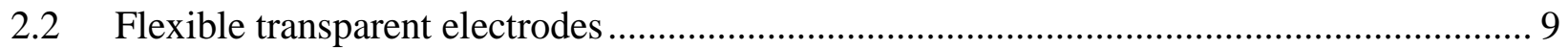

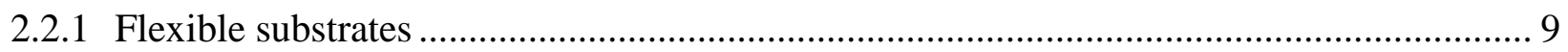

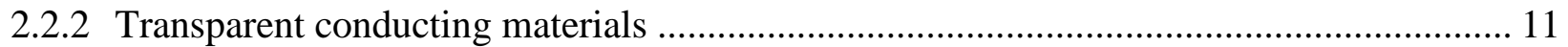

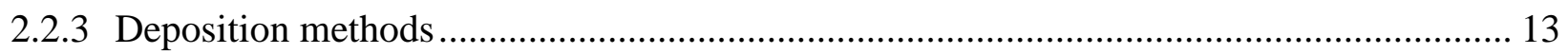

2.2 Degradation behavior of flexible transparent conducting materials ................................... 14

2.2.2 Electromechanical behavior of flexible transparent electrodes........................................... 15

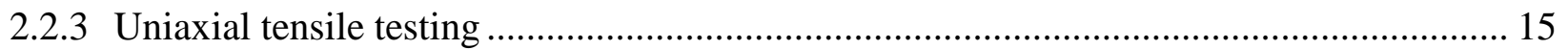




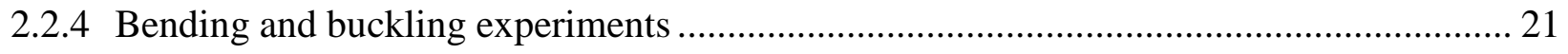

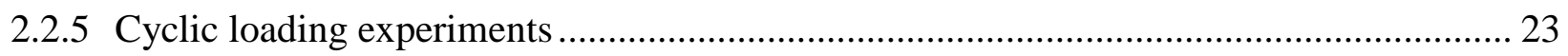

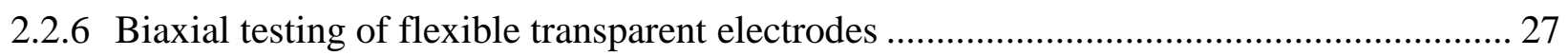

2.2.7 Wear, nanoscratch and nanoindentation experiments ...................................................... 28

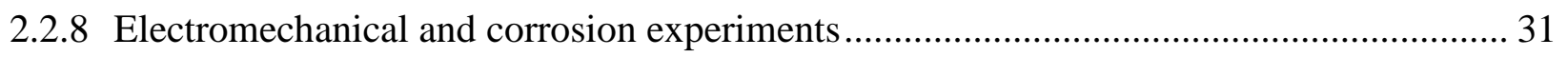

2.2.9 Tribo-corrosion behavior of transparent electrodes ........................................................... 33

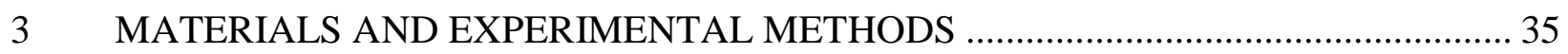

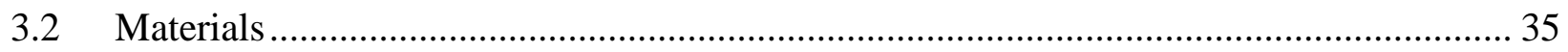

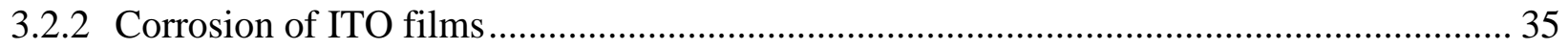

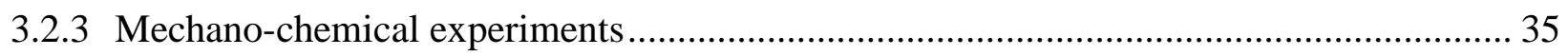

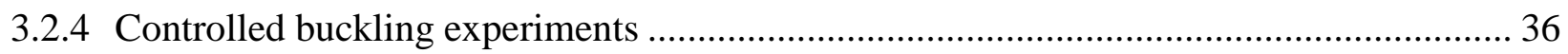

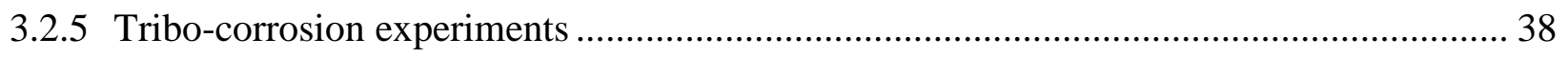

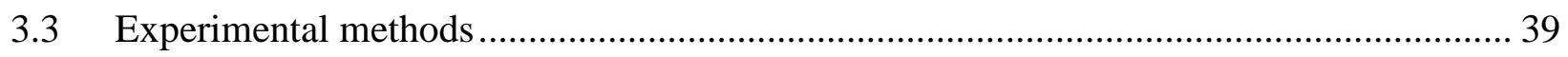

3.3.2 In-situ electrical measurements for corrosion experiments................................................ 39

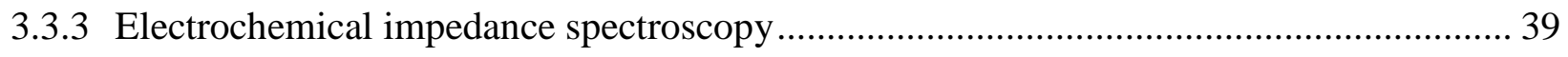

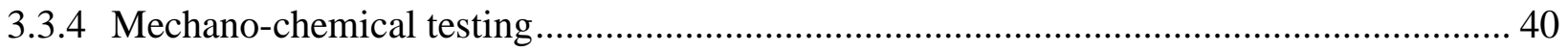

3.3.5 Controlled-buckling testing ......................................................................................... 43

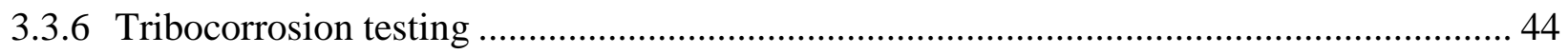

3.3.7 Surface characterization ................................................................................................. 46

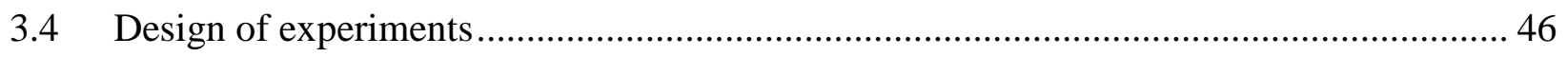

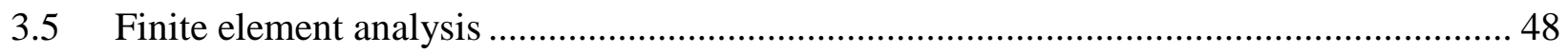

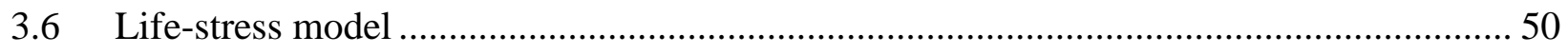

$4 \quad$ CORROSION OF FLEXIBLE TRANSPARENT ELECTRODES .................................... 51 


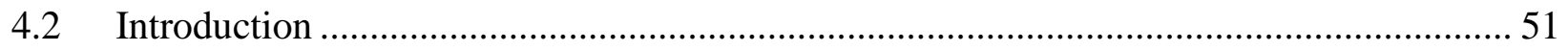

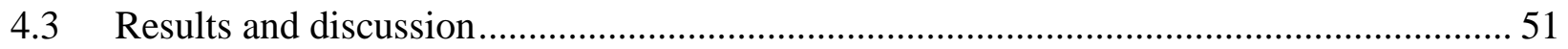

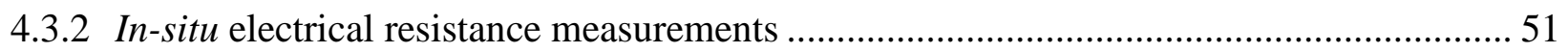

4.3.3 Open-circuit potential measurement ……….................................................................. 52

4.3.4 Potentiodynamic polarization measurement …………………........................................ 54

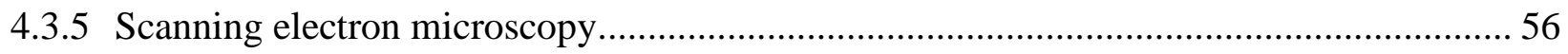

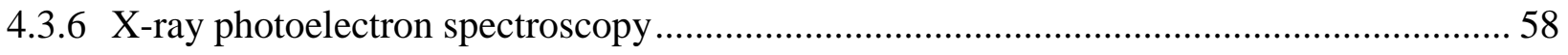

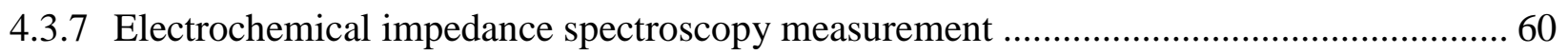

5 MECHANO-CHEMICAL DEGRADATION OF FLEXIBLE TRANSPARENT

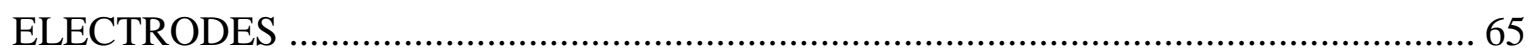

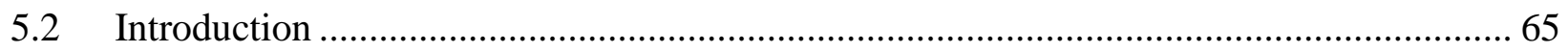

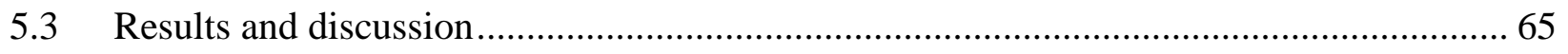

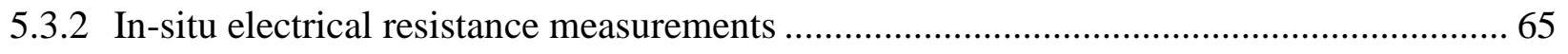

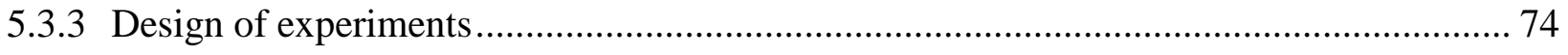

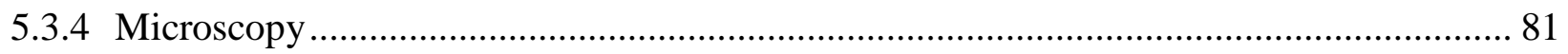

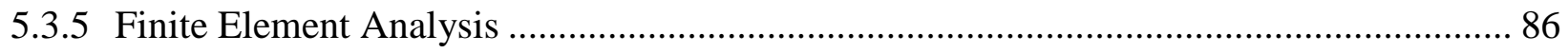

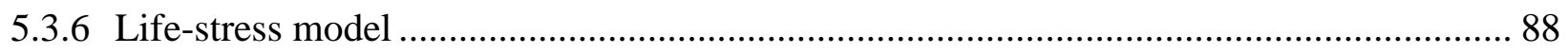

6 CONTROLLED-BUCKLING BEHAVIOR OF PATTERNED ELECTRODES.............. 91

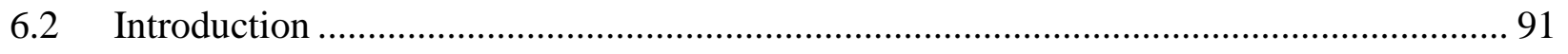

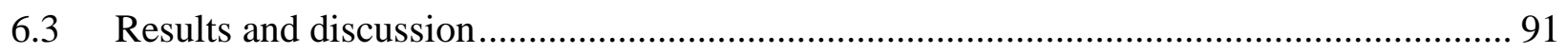

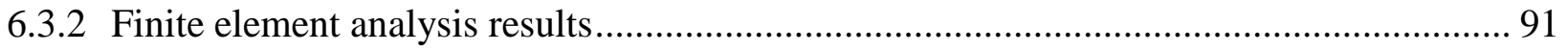

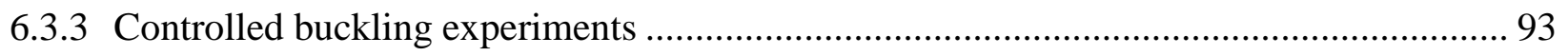

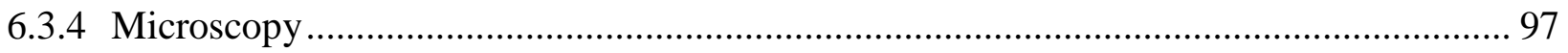




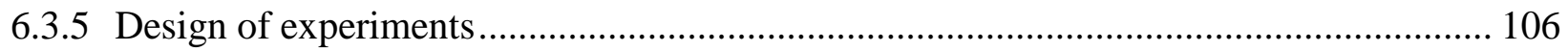

$7 \quad$ TRIBO-CORROSION OF FLEXIBLE TRANSPARENT ELECTRODES .................... 110

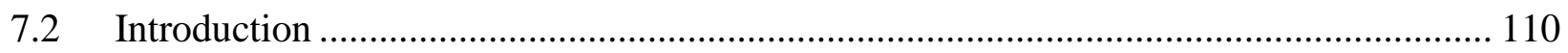

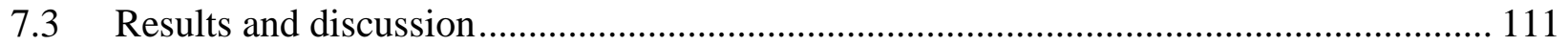

7.3.2 In-situ electrical resistance measurements ..................................................................... 111

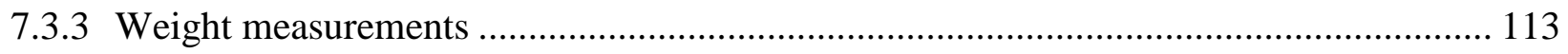

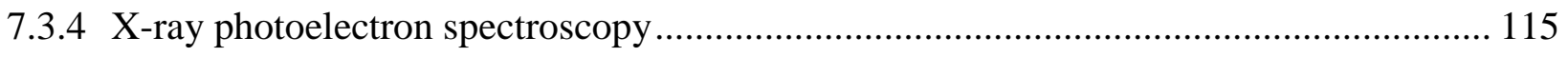

7.3.5 Electrochemical impedance spectroscopy ................................................................ 117

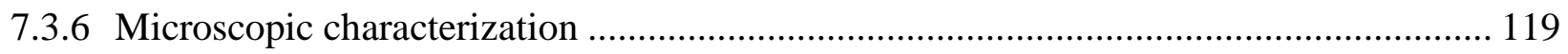

7.3.6.1 PTFE counterbody ……………………….................................................. 119

7.3.6.2 Multilayered sample surfaces ......................................................................... 121

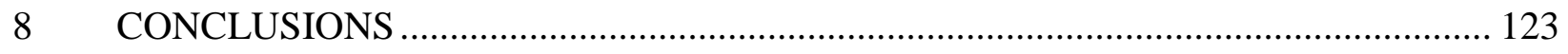

8.1 Conclusions from the corrosion studies ....................................................................... 124

8.2 Conclusions from the mechano-chemical investigations ................................................. 125

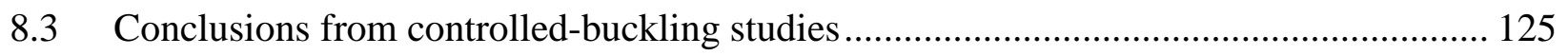

8.4 Conclusions from tribo-corrosion experimental work ….................................................. 126

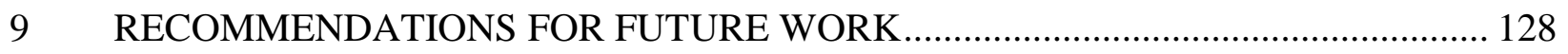

9.1 Mechanochemical studies of alternative electrodes ........................................................ 128

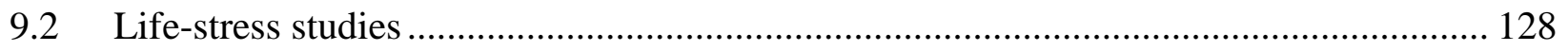

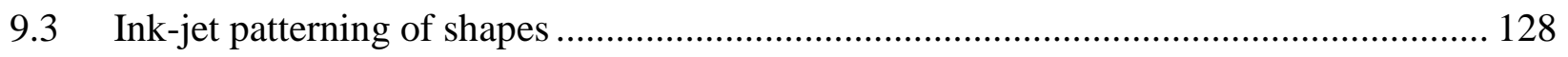

9.4 Insertion of Ag ink in ITO film crack openings ........................................................... 129

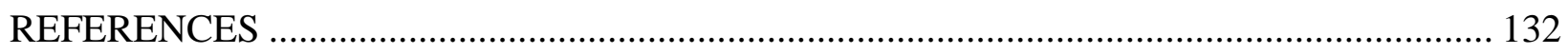




\section{LIST OF TABLES}

Table 2.1 Properties of common polymer substrates [29]........................................................ 11

Table 2.2 Electrical and optical properties of transparent electrodes [8] ................................. 12

Table 3.1 Materials used in tribo-corrosion experiments. ...................................................... 39

Table 3.2 Size of mandrel, specimen length, and approximate applied strain.............................. 42

Table 3.3 Factors and levels for DOE analysis of fatigue and fatigue-corrosion experiments. ... 47

Table 3.4 Factors and levels for DOE analysis of bending and bending-corrosion experiments. 47

Table 3.5 Factors and levels for DOE analysis of controlled-buckling experiments. ................... 48

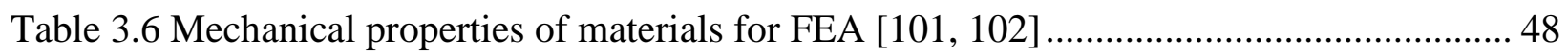

Table 3.7 Dimensions of specimen for FEA under bending......................................................... 49

Table 4.1 Potentiodynamic polarization parameters for ITO film after different immersion times

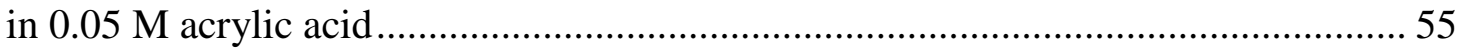

Table 4.2 Relative concentrations of indium species for ITO film after different immersion times

in $0.05 \mathrm{M}$ acrylic acid and electrochemical measurements......................................... 58

Table 4.3 The parameters of equivalent circuit obtained by fitting the experimental data of EIS for ITO film after different immersion times in $0.05 \mathrm{M}$ acrylic acid $\left(\mathrm{R}_{\mathrm{s}}=100 \Omega \mathrm{cm}^{2}\right)$.

Table 5.1 Analysis of variance (ANOVA) for fatigue and fatigue-corrosion ................................ 75

Table 5.2 Analysis of variance (ANOVA) for bending and bending-corrosion............................ 77

Table 5.3 Regression coefficients for fatigue and fatigue-corrosion.............................................. 80

Table 5.4 Regression coefficients for bending and bending-corrosion ......................................... 81

Table 5.5 Parameters for IPL-Weibull model............................................................................... 90 
Table 6.1 Calculated volume for different patterned shapes of ITO and their corresponding values of (a) COS-R, (b) COS-M, and (c) $\Delta R / R$ at COS-M. $A_{1} / A_{2}$ is the change in area

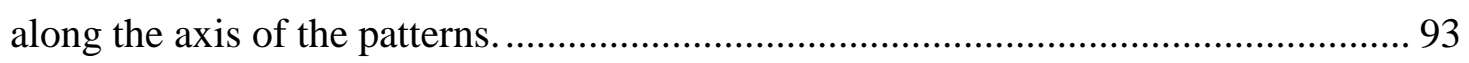

Table 6.2 Analysis of variance (ANOVA) for $\Delta \mathrm{R} / \mathrm{R}$ at COS-M under tensile buckling............. 106 Table 6.3 Analysis of variance (ANOVA) for $\Delta \mathrm{R} / \mathrm{R}$ at COS-M under compressive buckling. . 106 


\section{LIST OF FIGURES}

Fig. 1.1 A typical (a) organic solar cell and (b) liquid crystal display device stack.................... 3

Fig. 2.1 Schematic of tensile testing apparatus................................................................ 16

Fig. 2.2 Fragmentation morphology of the $\mathrm{SiO}_{\mathrm{x}}$ coating on the PET substrate at different strains

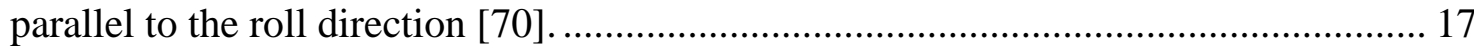

Fig. 2.3 Fractional change in resistance $(\Delta R / R)$ of ITO coated PET as a function strain $\varepsilon$ (left axis) experimental data for three thicknesses are shown as points ( $\bullet$ ) 105, ( $\square$ ) 42, and ( $) 16.8 \mathrm{~nm}$ and modeled fits with solid lines. Stress vs strain curve for ITO coated PET (right axis) is shown as a dotted line [71] ................................................. 18

Fig. 2.4 A schematic diagram showing bridging material at the PET substrate which results in an increasing but finite resistance with increasing strain [71]. 19

Fig. 2.5 Schematic of bending testing apparatus. 21

Fig. 2.6 Schematic of custom-built apparatus to monitor resistance during cyclic mandrel bending [80]. 24

Fig. 2.7 Fatigue test until catastrophic failure of ITO samples. Linear scale (left) and logarithmic scale (right) [80]. 25

Fig. 2.8 Photograph of the lab-made twisting test apparatus [83] ........................................ 26

Fig. 2.9 Schematic diagram of the operation of a resistive touch screen [16]......................... 29

Fig. 3.1 Different patterns of ITO coated PET films with (a) smaller size dimensions and (b) sharpness of corners. All dimensions are in $\mu \mathrm{m}$.

Fig. 3.2 Cross-sectional view of the sample under tribo-corrosion investigation. 38

Fig. 3.3 Schematic, 3D and exploded CATIA model (Dassault Systemes), and picture of the custom-built bending cyclic loading apparatus 
Fig. 3.4 Schematic of the custom-built manually operated buckling apparatus........................ 43

Fig. 3.5 Buckling test with built-in ends [74] ........................................................... 44

Fig. 3.6 Schematic of the custom-built reciprocating wear tester apparatus. ........................... 45

Fig. 4.1 Normalized electrical resistance vs. time for ITO film in acrylic acid of different

concentrations

Fig. 4.2 Open-circuit potential vs. time curves for ITO film after different immersion times in

$0.05 \mathrm{M}$ acrylic acid.

Fig. 4.3 Potentiodynamic polarization (Tafel fit) curves for ITO film after different immersion

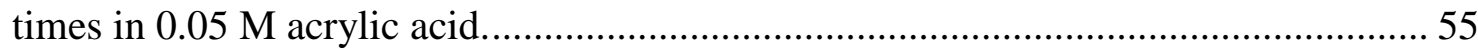

Fig. 4.4 SEM image of ITO film for (a) as received ITO, (b) $0 \mathrm{~min}$, (c) $90 \mathrm{~min}$, (d) $180 \mathrm{~min}$, (e) $270 \mathrm{~min}$, and (f) $360 \mathrm{~min}$ of immersion in $0.05 \mathrm{M}$ acrylic acid and after electrochemical

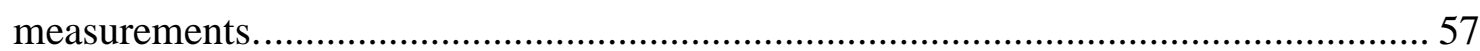

Fig. 4.5 XPS spectrum of ITO film for (a) as received ITO, (b) $0 \mathrm{~min}$, (c) $90 \mathrm{~min}$, (d) $180 \mathrm{~min}$, (e) $270 \mathrm{~min}$, and (f) $360 \mathrm{~min}$ of immersion in $0.05 \mathrm{M}$ acrylic acid and after electrochemical measurements. 59

Fig. 4.6 The equivalent circuit used to fit the impedance data for ITO film after different immersion times in $0.05 \mathrm{M}$ acrylic acid. 61

Fig. 4.7 Nyquist plot for ITO film after different immersion times in 0.05 M acrylic acid........ 61

Fig. 4.8 Bode frequency plot for ITO film after different immersion times in 0.05 M acrylic acid.

Fig. 4.9 Bode phase angle plot for ITO film after different immersion times in $0.05 \mathrm{M}$ acrylic acid. 64 
Fig. 5.1 Normalized electrical resistance versus experimental number of cycles and/or time for ITO-coated PET at applied strain and film thickness of: (a) $70 \mathrm{~nm}$ and 0.6\%, (b) 200 nm and 0.6\%, (c) $70 \mathrm{~nm}$ and 0.8\%, (d) $200 \mathrm{~nm}$ and 0.8\%, (e) $70 \mathrm{~nm}$ and 1.0\%, (f) 200 $\mathrm{nm}$ and $1.0 \%$, respectively. 67

Fig. 5.2 Critical number of cycles for fatigue and fatigue-corrosion of ITO-coated PET for film thickness of (a) $70 \mathrm{~nm}$ and (b) $200 \mathrm{~nm}$. 68

Fig. 5.3 Effect of strain on normalized electrical resistance for different testing conditions at 150,000 number of cycles for film thickness of (a) $70 \mathrm{~nm}$ and (b) $200 \mathrm{~nm}$. 70

Fig. 5.4 Effect of film thickness on normalized electrical resistance for different testing conditions at 150,000 number of cycles at applied strains (a) $0.6 \%$, (b) $0.8 \%$, and (c) $1.0 \%$ 72

Fig. 5.5 Normalized electrical resistance versus experimental time/number of cycles for CNTdeposited PET at applied strain of: (a) $0.6 \%$ and (b) $1.0 \%$ 73

Fig. 5.6 Main effects plot for change in electrical resistance for ITO films under fatigue and fatigue-corrosion. 76

Fig. 5.7 Interaction plot for change in electrical resistance for ITO films under fatigue and fatigue-corrosion. 76

Fig. 5.8 Main effects plot for change in electrical resistance for ITO films under bending and bending-corrosion. 78

Fig. 5.9 Interaction plot for change in electrical resistance for ITO films under bending and bending-corrosion. 78

Fig. 5.10 Normal probability plot for change in electrical resistance for ITO films under: (a) fatigue and fatigue-corrosion (b) bending and bending-corrosion. 79 
Fig. 5.11 Optical microscopy images of cracked $200 \mathrm{~nm}$ thick ITO films under fatigue. The corresponding number of cycle values is indicated on the images. Arrows indicate the

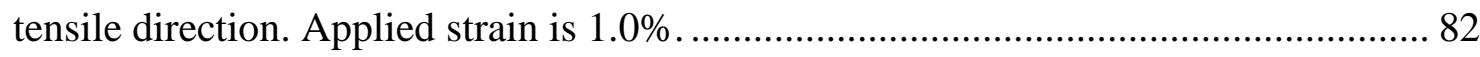

Fig. 5.12 Optical microscopy images of cracked $200 \mathrm{~nm}$ thick ITO films under fatigue-corrosion. The corresponding number of cycle values is indicated on the images. Arrows indicate the tensile direction. Applied strain is $1.0 \%$. 83

Fig. 5.13 Crack density versus number of cycles for $200 \mathrm{~nm}$ thick ITO films under fatigue and fatigue-corrosion. 84

Fig. 5.14 SEM image showing cracks on the surface ITO coated PET at $1.0 \%$ applied strain and 150,000 cycles under fatigue-corrosion. Arrows indicate the tensile direction. 85

Fig. 5.15 SEM image showing delamination of top coat on the surface CNT coated PET at $1.0 \%$ applied strain and 150,000 cycles under fatigue-corrosion. Arrows indicate the tensile direction. 86

Fig. 5.16 (a) and (b) Von Mises stress distribution, and (c) and (d) Von Mises strain distribution of 200 nm thick ITO-coated PET. 87

Fig. 5.17 (a) Effect of applied strain and (b) effect of thickness on the stress distribution of ITO film. 88

Fig. 5.18 Cycles to failure versus stress for the $200 \mathrm{~nm}$ thick ITO film under: (a) fatigue and (b) fatigue-corrosion. 89

Fig. 6.1 Von Mises stress distribution of (a) unpatterned and (b) square-patterned film under tensile buckling; (c) circle and (d) zigzag patterned film under compressive buckling. 92

Fig. 6.2 Normalized electrical resistance versus applied strain for the smaller size patterned ITO coated PET films under (a) tension and (b) compression. 94 
Fig. 6.3 Normalized electrical resistance versus applied strain for the larger size patterned ITO coated PET films under (a) tension and (b) compression. 95

Fig. 6.4 Crack onset strain from change in electrical resistance monitoring of ITO coated PET films. 96

Fig. 6.5 Optical microscopy images of circular-patterned ITO coated PET films under tensile buckling deformation mode showing crack initiation and propagation up to $6 \%$ strain. Arrows indicate the buckling direction. 98

Fig. 6.6 Optical microscopy images of circular-patterned ITO coated PET films under compressive buckling deformation mode showing crack initiation and propagation up to $6 \%$ strain. Arrows indicate the buckling direction.

Fig. 6.7 Optical microscopy images of square-patterned ITO coated PET films under tensile buckling deformation mode showing crack initiation and propagation up to $6 \%$ strain. Arrows indicate the buckling direction. 100

Fig. 6.8 Optical microscopy images of square-patterned ITO coated PET films under compressive buckling deformation mode showing crack initiation and propagation up to $6 \%$ strain. Arrows indicate the buckling direction. 101

Fig. 6.9 Optical microscopy images of zigzag-patterned ITO coated PET films under tensile buckling deformation mode showing crack initiation and propagation up to $6 \%$ strain. Arrows indicate the buckling direction. 102

Fig. 6.10 Optical microscopy images of zigzag-patterned ITO coated PET films under compressive buckling deformation mode showing crack initiation and propagation up to $6 \%$ strain. Arrows indicate the buckling direction. 103 
Fig. 6.11 SEM micrographs of patterned ITO coated PET films under (a) tensile and (b) compressive buckling deformation. Arrows indicate the buckling direction. 104

Fig. 6.12 Crack onset strain from in situ optical microscopy monitoring of ITO coated PET

films. 105

Fig. 6.13 Normalized electrical resistance at COS-M for ITO coated PEN films. 105

Fig. 6.14 Main effects plot for change in electrical resistance at COS-M for ITO films under: (a) tensile and (b) compressive buckling. 107

Fig. 6.15 Interaction plot for change in electrical resistance at COS-M for ITO films under: (a) tensile and (b) compressive buckling. 108

Fig. 6.16 Normal probability plot for change in electrical resistance at COS-M for ITO films under: (a) tensile and (b) compressive buckling. 109

Fig. 7.1 In-situ electrical resistance measurement against number of reciprocating cycles....... 111

Fig. 7.2 Critical number of reciprocating cycles for 20\% electrical resistance increase. 112

Fig. 7.3 Graph showing the weight loss of multilayer films versus number of reciprocating cycles in $1 \mathrm{M} \mathrm{NaCl}$ solution.

Fig. 7.4 Graph showing the weight loss of the PTFE sliding balls with respect to number of reciprocating cycles in $1 \mathrm{M} \mathrm{NaCl}$ solution.

Fig. 7.5 Typical x-ray photoelectron spectra for the case of Ag-Pd based ITO multilayer and increasing number of reciprocating cycles.

Fig. 7.6 Graph showing In atomic percentage concentration against number of reciprocating slides for all three samples sliding against PTFE balls in $1 \mathrm{M} \mathrm{NaCl}$ solution.

Fig. 7.7 Typical Nyquist plot for ITO/Ag-Au/ITO structures worn for up to 540 reciprocating cycles........ 118 
Fig. 7.8 Charge transfer resistance versus number of reciprocating cycles. Inset shows the equivalent circuit diagram used to model the multilayer structure.

Fig. 7.9 (a) Optical microscopy image of a PTFE ball surface sliding against an ITO/Ag/ITO surface in reciprocating motion for 4320 cycles, (b) 3-D surface profile of the same PTFE surface. Arrows indicate reciprocating motion direction. 120

Fig. 7.10 (a) Optical image of a worn ITO/Ag-Au/ITO sample surface for 540 reciprocating cycles. Scale bar is the same for both images and equal to $0.1 \mathrm{~mm}$ (b) Optical image of a worn ITO/Ag-Au/ITO sample surface for 3420 reciprocating cycles. The doubleheaded arrow is the same for both images and indicates reciprocating direction........ 122

Fig. 7.11 SEM image of a worn ITO/Ag-Au/ITO sample surface for 270 reciprocating cycles.

Double-headed arrow indicates the reciprocating direction. 122

Fig. 9.1 Schematic of insertion of Ag ink in ITO crack openings. 130 


\section{INTRODUCTION}

\subsection{Overview}

The next generation of optoelectronic devices is likely to include mechanical flexibility and it is expected to enable new applications which are not possible using currently available glass-based planar technology. The recent growing interest in the development of flexible optoelectronic materials has been spurred by the continuing evolution of large-area applications such as organic light emitting diodes (OLEDs), liquid crystal displays (LCDs), touch panels, solar cells, solid-state lighting (SSL), biomedical devices, and mechanical energy harvesters [16]. Such devices offer additional advantages, such as thin profiles, low-cost, large-area, light weight, conformability, robustness, and ease of roll-to-roll manufacturing [7].

Flexible optoelectronic device technology includes the utilization and integration of robust flexible substrates, transparent conducting materials, reflecting materials, thin film transistor and barrier layers [7]. In addition, processes such as roll-to-roll manufacturing and various coating technologies must also be further developed and optimized [7]. Moreover, in order for flexible optoelectronic devices realize their full commercial potential, the device degradation issues involved when the device is stressed, bent, or generally deformed during manufacturing and/or in-service conditions have to be investigated.

One of the most critical components of flexible devices is the electrode component. Flexible anodes are usually a hybrid system of transparent conducting layer and a flexible substrate. Polymers are routinely used as the substrate material. This is because of their high optical transparency and mechanical flexibility when compared to thin glass and metal foils. 
There are various candidate materials for transparent conducting layers such as metallic nanowires ( $\mathrm{Al}, \mathrm{Ag}$, and $\mathrm{Cu}$ ), semiconductors (indium tin oxide, indium zinc oxide, and aluminum zinc oxide), and carbon-based (conductive polymers, carbon nanotubes, and graphene) [8]. However, transparent conducting oxides (TCO) are still enjoying a dominant role. This is because of their excellent combination of electrical and optical properties. Although CNT and graphene are being developed as promising alternatives due to their mechanical flexibility and durability, for most display applications indium tin oxide (ITO) still is a prevailing material [8].

In optoelectronic devices involving TCO layers deposited on polymer substrates such as polyethylene terephthalate (PET), the structural integrity of the anode component in the device structure represents a major challenge, to both manufacturing processes and in-service conditions. This is due to thermal and mechanical mismatches between the ceramic layer (ITO) and the organic substrate [9]. In particular when the high modulus ITO adheres to the polymer substrate there is a significant elastic modulus mismatch which can cause cracking and/or delamination of the ITO layer [10]. This may lead to electrical and optical degradation of the device [11]. Therefore the mechanical behavior of the electrode component under different types of loadings is of paramount importance.

\subsection{Motivation}

The motivation for this work is to investigate the electrochemical, electromechanical, and mechanochemical degradation behavior of flexible transparent electrodes which are used as anodes in flexible optoelectronic devices. The electrode may corrode due to acid-containing pressure sensitive adhesive and/or acid treated components present in the device stacks (Fig. 
1.1). Also, structural failure may occur due to external applied loading. It is therefore important to conduct an experimental-based study of the electrodes under different conditions such as corrosion, bending, fatigue, bending-corrosion, and tribo-corrosion. Such conditions simulate induced stress and failure both during manufacturing and in-service conditions.

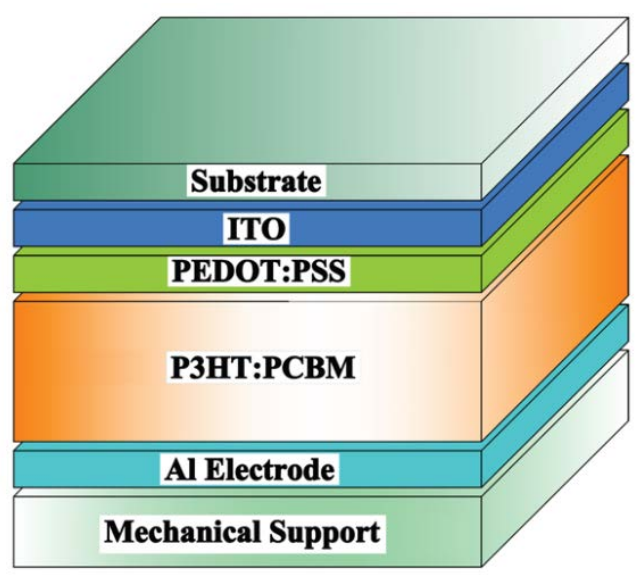

(a)

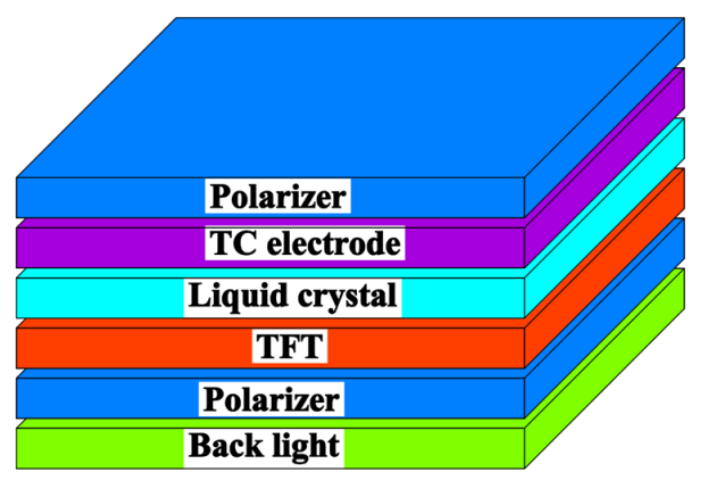

(b)

Fig. 1.1 A typical (a) organic solar cell and (b) liquid crystal display device stack. 
This work aims in enhancing the current understanding of the effects and synergies of corrosion, film thickness, applied strain, and number of cycles on the electrical, mechanical, and corrosion-resistant properties of such electrodes when they are subjected to various degradation conditions.

\subsection{Objectives}

The objective of this study is to investigate the degradation behavior of flexible transparent electrodes using electrochemical, electromechanical, and mechanochemical characterization protocols. Degradation mechanisms of three types of conducting layers, ITO, CNT, and ITOAg/Ag alloy-ITO multilayers on polymer substrates, are investigated. Changes in structural and electrical properties are studied.

The specific objectives are:

- Development of new testing protocol and apparatus for the characterization of electrodes in order to simulate fatigue-corrosion degradation during in-service conditions.

- Investigation of corrosion, bending, fatigue, bending-corrosion, and fatigue-corrosion behavior of ITO and CNT using in-situ electrical resistance measurements and ex-situ surface characterization methods.

- Controlled buckling behavior of various patterned ITO shapes on PET substrates to identify critical strains and potential failure mechanisms. 
- Tribo-corrosion investigation of ITO-Ag/Ag alloy-ITO multilayers in order to investigate the suitability of such model systems as durable components in solar energy and energy-efficient applications.

- Simulation of stress distribution and stress intensity of the electrodes under different deformation modes using finite element method.

- Statistical analysis based on design of experiments methods to identify the effects of factors such as corrosion, applied strain, film thickness, and number of bending cycles and/or time on the change in electrical resistance of the electrodes.

- Life-stress analysis using the stress values obtained from finite element analysis and cycles to failure data from mechanochemical experiments for life prediction.

\subsection{Structure of dissertation}

This thesis is organized into nine chapters.

Chapter 1 provides brief overview related to this research. Objectives and organization of the thesis are also presented.

Chapter 2 reviews literature covering several topics that are related to this research work. The topics include previous degradation studies mainly of transparent conducting coatings on polymer substrates for optoelectronic device applications.

Chapter 3 provides details of the materials and methods used in this research. A wide range of experimental methods is presented. Finite element modeling, design of experiments method, and life-stress analysis are also discussed. 
Chapter 4 presents the results and discussion of electrochemical degradation studies of ITO on PET substrate in acrylic acid solution. Such results are used as an input for chapter 5.

Chapter 5 discusses the results of mechano-chemical experiments conducted using flexible transparent electrodes. In addition, stress intensity of the films is predicted using finite element modeling. Furthermore, design of experiments is employed in order to investigate the effects of factors affecting the reliability of transparent electrodes. Finally, life-stress analysis is presented. Chapter 6 is dedicated to electromechanical studies of patterned ITO on PET substrates. Experimental and finite element modeling results of micron-sized shapes including squares, circles, and zigzag-based structures will be reported.

Chapter 7 is, mainly, related to the discussion of tribo-corrosion experimental results on metallic/ITO multilayers.

Chapter 8 is a summary of the results and presents the conclusions drawn from this project. Chapter 9 is providing some recommendations for future work. 


\section{LITERATURE REVIEW}

As outlined in the introduction, this project proposes to investigate the degradation behavior of flexible transparent electrodes and aids towards an understanding of the effects of different factors on the electrical and structural integrity of such electrodes. The first section covers a brief description of optoelectronic device applications. The next section discusses the most common types of transparent electrodes. Finally, the last section provides a detailed review of various experimental methods used to assess different failure modes.

\subsection{Flexible optoelectronic devices}

Flexible transparent conducting films are essential components for a large variety of optoelectronic devices, acting as transparent electrical contacts or electrodes. By integrating them into a thin, flexible sheet of polymer, one could obtain a durable, lightweight product suitable for many applications in the growing market of cell phones, personal digital assistants (PDAs) flat panel displays, touch panels, thin film solar cells, SSL, biomedical, energy harvesting, and electrochromic devices [12, 13].

\subsubsection{Flexible displays}

Unlike rigid glass-based displays, flexible displays are thin and light weight. Also, flexible displays can be fabricated by a roll-to-roll process, which is potentially low cost. They include, but are not limited to, LCDs, OLEDs, plasma display panels, and field emission displays 
[14]. Flexible display system includes robust flexible substrates, conducting transparent electrodes, electro-optic and reflecting materials, inorganic and organic electronics, and packaging technologies [7].

\subsubsection{Touch panels}

Touch panel mobile devices are becoming more popular with both manufacturers and users. As there is no need for physical keyboard to take up space on the device, they can have larger screens which can be used more flexibly, meaning a better display as required, for example pinching, zooming, and rotating from portrait to landscape [15]. There are a number of touch panel technologies but the two most widely used rely on transparent conductive oxides (TCOs). For capacitive touch panels a TCO layer is deposited on a substrate and a conducting pattern is printed around the edge of the substrate. The conducting pattern is designed such that when voltages are applied to the corners a uniform electric field is produced on the screen surface. When the screen is touched with a finger, a current is drawn and the position of the touch is detected. In a resistive touch panel a TCO coated glass substrate and a similarly coated polymer substrate are separated by dielectric spacers. The resistance between the contacts on the two substrates is monitored and when a touch forces the substrates to contact, the change in resistance between the reference points is measured. The resistance measurements are then used to calculate the position of the touch [16].

\subsubsection{Solid state lighting}


Flexible SSL that uses semiconductor technology based on LEDs, OLEDs, or polymer light-emitting (PLEDs) is replacing traditional lighting technologies such as incandescent light bulbs, fluorescent tubes, or compact fluorescent lamps [17, 18]. SSL offer significant advantage such as energy efficiency, compact profile, breakage resistance, light weight, increased color quality, and long life. Because of SSL's flexibility and durability, they are seeing applications in areas such as displays, buildings (interior and exterior), transport (automotive, trains, aerospace), and telecommunications [19-22].

\subsubsection{Solar cells}

There is currently considerable interest in flexible solar cells due to their light weight, robust profile, as well as their ease of fabrication using roll-to-roll based thin film technologies [23- 25]. Flexible solar cells require a transparent electrode to allow light to enter the photovoltaic layer [26]. At the front of a solar cell, the TCO layer acts as an electrical contact as well as a window to allow light to pass through the photovoltaic (PV) absorber layer. At the back of the cell, the TCO usually acts as an interfacial layer to improve the contact resistance between the PV absorber and a metallic reflector and also as an optical coupling layer by improving the refractive index matching. In bi-facial solar cells, the back contact must both act as an electrical contact and as a window layer to let light through the back of the PV absorber layer [27].

\subsection{Flexible transparent electrodes}

\subsubsection{Flexible substrates}


Due to their flexibility, robustness, and cost, polymer substrates are preferred than thinglass and metal foils in flexible optoelectronic devices. Thin glass is optically clear, can be made very smooth and impermeable to water [28]. Also, it exhibits relatively low thermal expansion coefficients. However, it is susceptible to cracking and breakage. Metal foils such as $\mathrm{Al}, \mathrm{Cu}$ and stainless steel can be used as a substrate material. However, they have low optical transparency. Also, they should be coated with an insulator to prevent short circuit [28]. On the other hand, the mechanical flexibility of polyester-based substrates such as polyethylene terephthalate (PET) [29], polyethylene naphthalate (PEN) [12], polyethersulfone (PES) [30], and polycarbonate (PC) [31] make them ideal candidates for roll-to-roll manufacturing. However, most polymer substrates exhibit low thermal stability, a high susceptibility to chemical degradation, relatively high surface roughness, and high permeability to oxygen and water vapor. PET and PEN are prepared by a process whereby an amorphous cast is drawn in both the machine and transverse directions [32]. The biaxially oriented film is then heat set to crystallize the film. The polymers are usually heat stabilized during fabrication to control shrinkage. Also, in order to achieve high surface quality required for depositing subsequent layers, a planarizing coating is usually applied [32, 33]. PET has a Young's modulus of 5.3 GPa. On the other hand, PEN has a modulus of 6.1 GPa $[34,35]$. Polymers with low stiffness and high $T_{\mathrm{g}}$ are desirable for roll-to-roll process. During high temperature roll-to-roll processes above $\mathrm{T}_{\mathrm{g}}$, polymer substrates undergo a molecular relaxation process which alters the physical and mechanical properties of the film [36]. Table 2.1 illustrates comparisons of common polymers for optoelectronic application. 
Table 2.1 Properties of common polymer substrates [29].

\begin{tabular}{lllllll}
\hline & PET & PEN & PC & COC & PES & PI \\
\hline Thickness $(\mathrm{mm})$ & 0.1 & 0.1 & 0.1 & 0.1 & 0.1 & 0.1 \\
Total light transmittance $(\%)$ & 90.4 & 87.0 & 92.0 & 94.5 & 89.0 & $30-60$ \\
Retardation $(\mathrm{nm})$ & Large & Large & 20 & 7 & $<10$ & Large \\
Reflactive index & 1.66 & 1.75 & 1.56 & 1.51 & 1.6 & - \\
Glass transition temperature $\left({ }^{\circ} \mathrm{C}\right)$ & 80 & 150 & 145 & 164 & 223 & $300<$ \\
Coefficient of thermal expansion $\left(\mathrm{ppm} /{ }^{\circ} \mathrm{C}\right)$ & 33 & 20 & 75 & 70 & 54 & $8-20$ \\
Water absorption ratio $(\%)$ & 0.5 & 0.4 & 0.2 & $<0.2$ & 1.4 & $2.0-3.0$ \\
$\mathrm{H}_{2} \mathrm{O}$ barrier $\left(\mathrm{g} / \mathrm{m}^{2} /\right.$ day) & 9 & 2 & 50 & - & 80 & - \\
\hline
\end{tabular}

\subsubsection{Transparent conducting materials}

Despite growing efforts to replace them, transparent conducting oxide (TCO) layers deposited on polymer substrates are still enjoying a dominant role as the electrode component. The diverse nature of the materials integrated into these devices, including semiconductors, polymers, ceramics, glass, and metals have necessitated the need for TCO materials with enhanced performance, processability and even morphology [37]. Transparent conducing materials exhibit a remarkable combination of optical transparency and electrical conductivity [38]. The most popular TCOs are tin-doped indium oxide (ITO) and aluminum, gallium or indium-doped zinc oxide (AZO, GZO or IZO) [39- 41]. ITO dominates the TCO market and is expected to continue dominating the market for the next few years. AZO has been used as an alternative transparent electrode [42] and exhibits a resistivity as low as $8.4 \times 10^{-4} \Omega \mathrm{cm}$ with optical transmittance approximately equal to $80 \%$ [43]. However, harsh environmental conditions such as annealing in air or humidity damping significantly increases electrical resistivity and surface degradation [44]. Recently, carbon nanotube (CNT) and graphene films are considered as a promising alternative to TCOs because they are mechanically strong and flexible [45]. Other alternative transparent conductive materials are conductive polymers. 
Poly(3,4-ethylenedioxythiophene) poly(styrenesulfonate) PEDOT:PSS is more mechanically flexible than ITO. However, it is less conductive [46]. More recently, a resistivity as low as $3.3 \mathrm{x}$ $10^{-4} \Omega \mathrm{cm}$ was observed with sulfuric acid treated PEDOT:PSS on glass and PET substrates [47]. Furthermore, ITO-metal-ITO multilayer structures have been also studied as flexible transparent electrode in order to take the advantage of mechanical properties of metals [48]. Table 2.2 illustrates the electrical and optical properties of common transparent electrodes.

Table 2.2 Electrical and optical properties of transparent electrodes [8].

\begin{tabular}{|c|c|c|c|c|c|c|}
\hline Material class & Material & $\begin{array}{l}\text { Bulk resistivity } \rho \\
(\mu \Omega \mathrm{cm})\end{array}$ & $\begin{array}{l}\text { Carrier concentration } \\
n\left(10^{21} \mathrm{~cm}^{-3}\right)\end{array}$ & $\begin{array}{l}\text { Mobility } \\
\mu\left(\mathrm{cm}^{2} \mathrm{~V}^{-1} \mathrm{~s}^{-1}\right)\end{array}$ & $\begin{array}{l}\text { Plasma wavelength } \\
\lambda(\mu \mathrm{m})\end{array}$ & $\sigma_{d c .} / \sigma_{o p t}$ \\
\hline \multirow[t]{3}{*}{ Metals } & Al & 2.5 & 180 & 14 & 0.08 & 75 \\
\hline & $\mathrm{Ag}$ & 1.5 & 58 & 72 & 0.14 & 215 \\
\hline & $\mathrm{Cu}$ & 1.55 & 85 & 48 & 0.11 & 75 \\
\hline \multirow[t]{5}{*}{ Semiconductors } & ITO & $>100$ & $<3$ & $=20-100$ & 0.77 & $120-240$ \\
\hline & $\mathrm{SnO}_{2}$ & $>500$ & $<1.0$ & $\approx 15-50$ & 1.1 & \\
\hline & $\mathrm{ZnO}$ & $>200$ & $<1.0$ & $=1-5$ & 1.1 & \\
\hline & $\mathrm{TiO}_{2}$ & $>500$ & $<4$ & $\approx 10-70$ & 1.27 & \\
\hline & $(\mathrm{Si})$ & $>100$ & $<5$ & & 0.95 & \\
\hline \multirow[t]{3}{*}{ Carbon } & Polymers & $>700$ & $<10 ?$ & $<1 ?$ & $0.8 ?$ & \\
\hline & SWNT & $>150$ & $3 \times 10^{-4}$ & $<10$ & 60 & $6-14$ \\
\hline & Graphene & $>5$ & $<0.3$ & $<5,000$ & 1.9 & 70 \\
\hline
\end{tabular}

\subsubsection{Indium tin oxide}

ITO is a wide bandgap (3.5-4.3 eV) n-type, highly degenerate semiconductor which has a relatively low electrical resistivity $\left(\sim 10^{-4} \Omega \mathrm{cm}\right)$ and a high transparency $(>90 \%)$ to visible light [49, 50]. ITO films conduct electricity through a tin-doped $\operatorname{In}_{2} \mathrm{O}_{3}$ lattice. The addition of tin causes the substitution of $\mathrm{Sn}^{4+}$ with $\mathrm{In}^{3+}$, creating more electrons by means of the n-type donor mechanism [50]. At high levels of Sn content (>10 mol \%), n-type doping is the dominant factor in raising electrical conductivity, while oxygen vacancies play a role in determining the conductivity at lower Sn levels [51]. In crystalline form, the semiconducting ceramic contains 
free electrons generated within the grains and electrons trapped at the grain boundaries [52]. On the other hand, amorphous ITO is less conductive. The low temperature sputtering of ITO on flexible polymer substrates often leads to an amorphous microstructure [53]. ITO has a coefficient of thermal expansion of $7.2 \times 10^{-6}{ }^{\circ} \mathrm{C}^{-1}$ [54]. Thin films of ITO are commonly deposited on flexible polymer substrates using electron beam evaporation or sputter deposition methods [4, 55- 57].

\subsubsection{Carbon nanotubes}

Single-walled carbon nanotubes (SWNTs) are a class of material with molecular structure being visualized as graphene sheets rolled-up to certain directions into a seamless cylinder [58]. Transparent, conductive thin films of CNTs are emerging as a promising alternative to traditional transparent conductors such as ITO in low-cost solution-processed applications due to the natural abundance of carbon, amenability to spraying and printing, and good wetting properties [59]. Applications requiring optical transparency between $80-95 \%$ require a CNT film thickness between 10 and $50 \mathrm{~nm}$. Therefore, very little mass of CNT material is required to achieve the desired film thickness $\left(5-25 \mathrm{mg} / \mathrm{m}^{2}\right)$ and sheet resistance, $\mathrm{R}_{\mathrm{s}}=110 \Omega / \mathrm{sq}[60,61]$. Also, CNTs exhibit superior mechanical flexibility $[62,63]$.

\subsubsection{Deposition methods}

Transparent conductive electrodes can be deposited on polymer substrates using various

methods such as, chemical vapor deposition [64], DC or RF magnetron sputtering [65, 31], and 
pulsed laser deposition [66]. Magnetron sputtering is one of the most common deposition methods because it provides high sputtering rates and can yield large area coatings [30]. During magnetron sputtering, a magnetic field is applied around the target and a glow discharge produces an electron-trapping effect resulting in an increased collision rate between the electrons and the sputtering gas molecules. This leads to increased deposition rates and sputtering gas pressures as low as $10^{-5}$ torr $[67,68]$.

\subsection{Degradation behavior of flexible transparent conducting materials}

The performance of the transparent electrode under mechanical and corrosion conditions represents a challenge in terms of the device functionality. For example, a flexible transparent electrode may lose its structural integrity, and therefore functionality, when the device is conformed to various shapes during manufacturing and in operation. Also, acid containing adhesives and/or acid treated components in the device stack may degrade the transparent conducting layer. Furthermore, the coefficient of thermal expansion (CTE) between the conducting layer and the polymer substrate may lead to loss of functionality of the device under temperature changes during both processing and in-service. The reliability of flexible transparent electrodes under different degradation mechanisms can be evaluated by electromechanical and/or corrosion testing techniques. Such experiments are usually accompanied by in-situ monitoring and observation of both the change in conductive coating's electrical resistance and the crack formation on its surface. A commonly-used comparative measure of failure initiation is the crack onset strain (COS). The COS can be determined using a direct observation of crack formation on 
the coating's surface under microscopy or when the electrical resistance of the coating has increased by $10 \%$. This value is arbitrarily chosen for flexible devices [69].

\subsubsection{Electromechanical behavior of flexible transparent electrodes}

One of the major concerns regarding the development of flexible electronics is the need to design devices with a performance that does not degrade due to the application of external mechanical stresses. The stresses in the multilayer inorganic/organic composite resulting from mechanical loading may cause either cohesive failure of the inorganic layer, or adhesive failure between the layer and the substrate [69]. In order to assess such failures, a variety of experimental procedures has been proposed and developed by several researchers over the years.

\subsubsection{Uniaxial tensile testing}

In a uniaxial tensile test, a dog-bone shaped or a strip specimen is loaded in tension and in-situ electrical resistance measurements and optical images can be acquired to evaluate the degradation of the conductive coating (Fig. 2.1). 


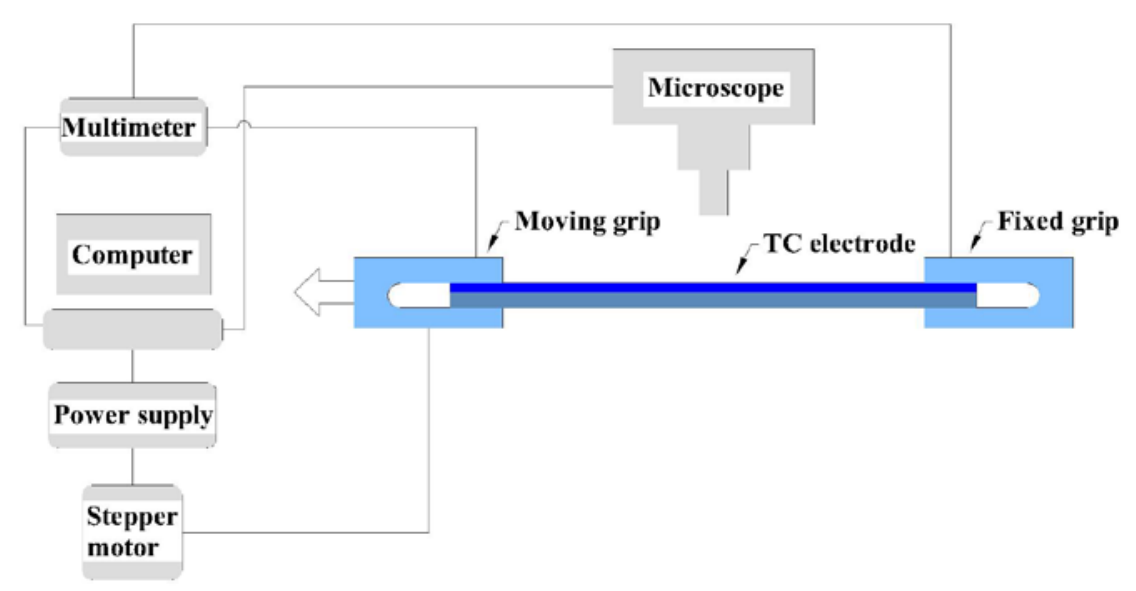

Fig. 2.1 Schematic of tensile testing apparatus.

Leterrier et al. [70] used uniaxial testing of a $100 \mathrm{~nm}$ silicon oxide layer deposited on PET substrate to investigate the effect of substrate properties on coating's tensile fragmentation process (Fig. 2.2). The COS of the coating was identified, using optical microscopy, at 1.2\% strain. At about 6\% strain, transverse cracking of the fragments is initiated. These cracks develop parallel to the straining direction due to the lateral contraction of the substrate resulting from Poisson's effects. At 10\% strain, tertiary cracks appear which are parallel to the primary cracks and are stopped by the secondary cracks. They do not therefore cross the full sample width. At 10\% strain, partial debonding appears along the secondary cracks. The fragments tend to overlap, which enhances debonding. The dissociation of the oxide layer from the substrate is at the origin of the interruption of the tertiary cracks. At this stage, the stress concentration at the crack front will relax over the debonded length and is no longer transmitted directly to the adjacent fragment. They also observed, upon unloading samples strained to less than $4 \%$ nominal strain, strain recovery leads to the closure of coating cracks. 


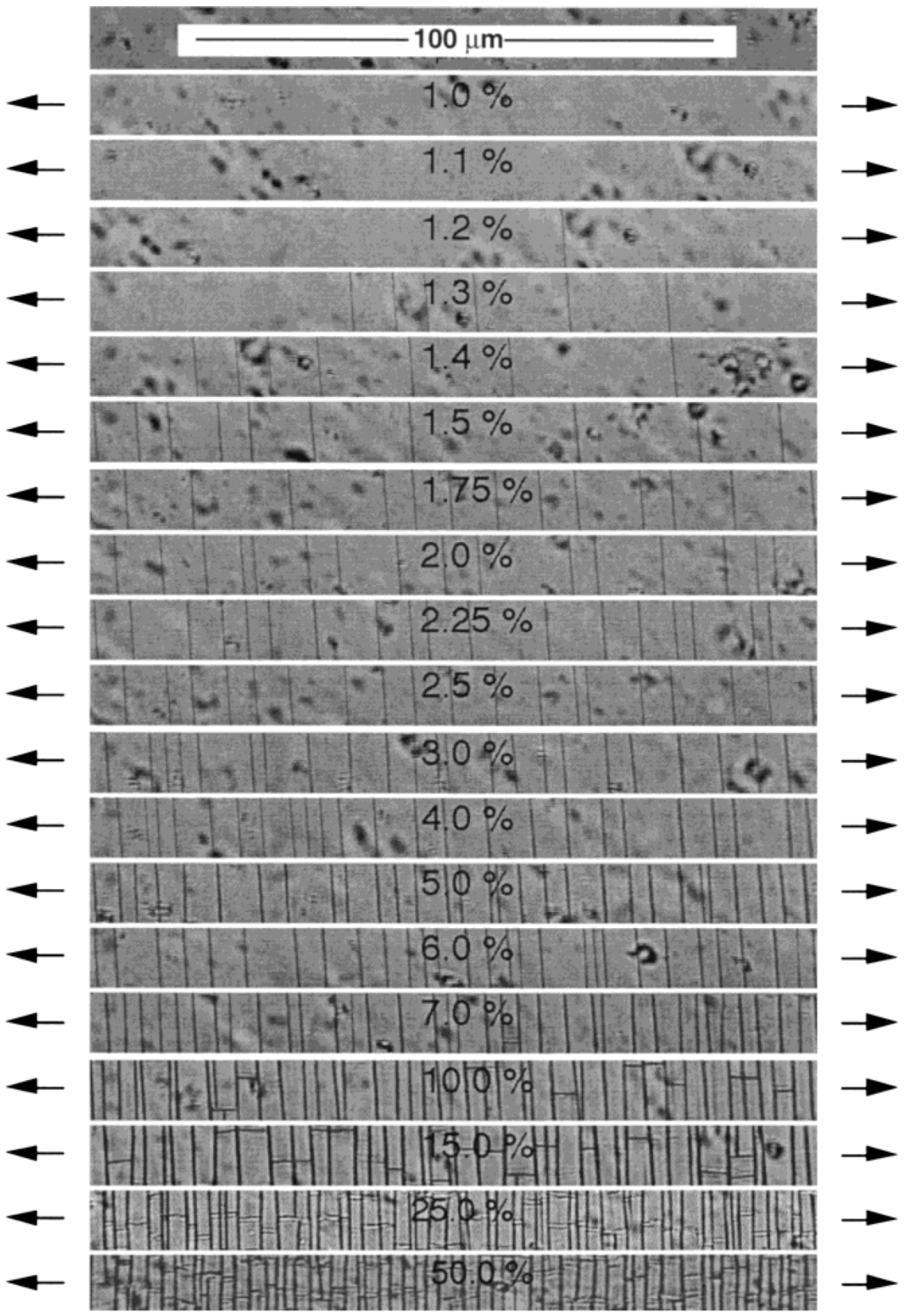

Fig. 2.2 Fragmentation morphology of the $\mathrm{SiO}_{\mathrm{x}}$ coating on the PET substrate at different strains parallel to the roll direction [70]. 
In addition, Cairns et al. [71] used a miniature tensile testing machine attached to a microscope stage to investigate the electromechanical behavior of ITO films on PET. The increase in electrical resistance is related to the number of cracks in the conducting layer which depends upon the applied strain and the film thickness. The onset of cracks between $2 \%$ and 2.5\% strain correlates with the sudden increase of the resistance of the sample (Fig. 2.3).

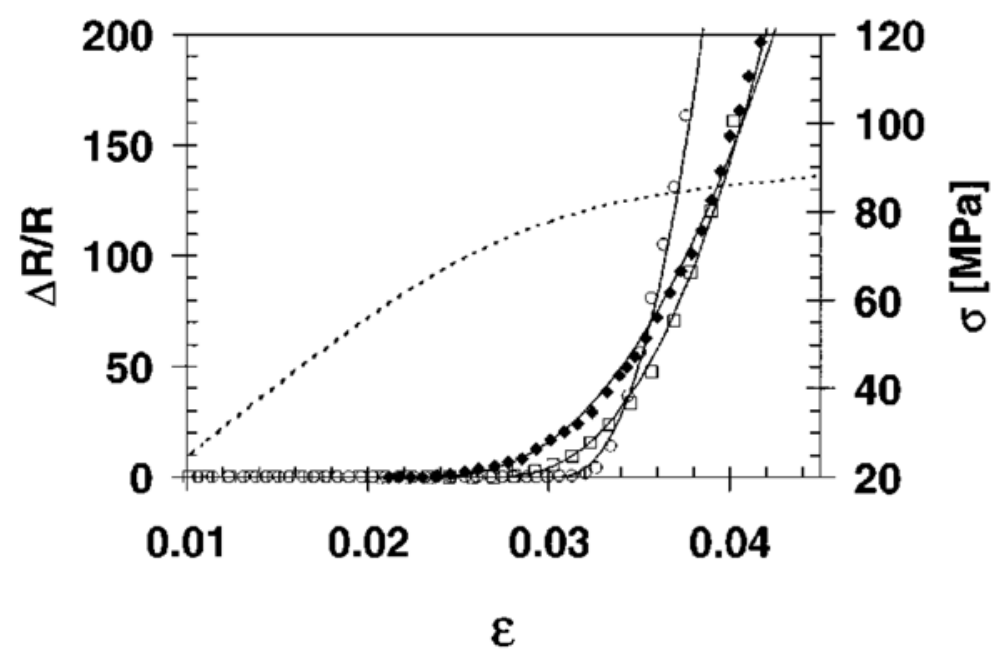

Fig. 2.3 Fractional change in resistance $(\triangle R / R)$ of ITO coated PET as a function strain $\varepsilon$ (left axis) experimental data for three thicknesses are shown as points $(\bullet) 105$, ( $\square$ ) 42, and (०) 16.8 $\mathrm{nm}$ and modeled fits with solid lines. Stress vs strain curve for ITO coated PET (right axis) is shown as a dotted line [71].

The cracks traverse the full width of the section but the electrical resistance remains finite. They proposed that the reason for the increasing, but finite in nature, of the resistance is the conduction between fragments due to a ductile layer at the interface between the ITO and the PET. In addition, they proposed a simple model (Fig. 2.4) that describes the increasing electrical resistance for a cracked ITO layer. In this model, at each crack there is a non-conductive gap 
between the two sides of the conductive layer, but there is a small conducting interfacial layer bridging it. As strain increases, the width of the crack increases and new cracks will nucleate and propagate.

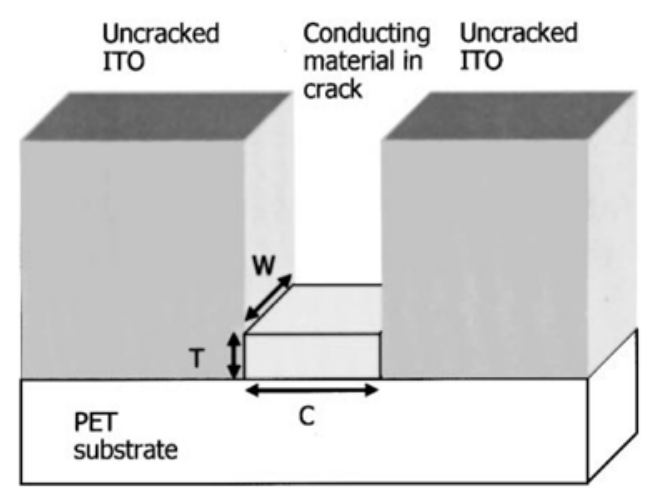

Fig. 2.4 A schematic diagram showing bridging material at the PET substrate which results in an increasing but finite resistance with increasing strain [71].

Furthermore, Leterrier et al. [72] investigated the mechanical integrity of ITO thin films sputtered onto a high temperature aromatic polyester by means of tensile experiments equipped with electrical measurement, and an optical microscope for in-situ observations. They observed that damage of ITO coatings under tensile load initiated at defect sites such as pin-holes, and propagated in a stable manner until the crack length was of the order of $100-500$ times the coating thickness. At that stage, the increase in electrical resistance was equal to approximately $10 \%$, after which crack propagation became unstable and electrical resistance increased by many orders of magnitude. Therefore, the loss of functional performance of the ITO coated polymers was controlled by crack propagation features, rather than by crack initiation. Also, homogenous ITO structures in the amorphous state as well as a fine grained polycrystalline structure were both found to be beneficial for the cohesive properties of ITO. This is due to the fact that fine 
grained structures are associated with less stress concentrators. They also found that an improved surface quality of the polymer substrate due to planarization was a major factor to increase the cohesive properties of ITO films. This is because a planarization layer helps to suppress superficial flaws such as scratches on the polymer surface which may lead to stress concentrations in the interfacial region between the ITO layer and the polymer substrate.

Furthermore, Cairns and Crawford [10] studied the electromechanical behavior of ITOcoated PET under tensile loading. The electrical resistance and the crack evolution as a function of uniaxial strain is monitored in-situ. Cracking was first observed at $2.3 \%$ nominal strain, and then the number of cracks increased rapidly to $2.6 \%$ strain. At $6 \%$ strain, transverse cracking occurred due to lateral contraction. Finally, at $10 \%$ strain, tertiary cracks appeared parallel to the primary cracks, but they were stopped by the secondary cracks.

Also, Hamasha et al. [73] investigated the behavior of sputtered ITO on PET substrate under uniaxial stretching. Two different sheet resistances, 100 and $60 \Omega / s q$ specimens were stretched up to $15 \%$ strain under three different strain rates, $0.01,0.1$, and $1.0 \mathrm{~min}^{-1}$. The crack development was monitored using an optical microscope. For both sheet resistances and different stain rates, cracks initiated perpendicular to the tensile loading and propagated towards the edges of the sample at $4 \%$ applied strain. At $8 \%$ strain, transverse cracks initiated and propagated between the original cracks. High crack intensity was found for high sheet resistance specimens and at a higher strain rate.

Finally, Sierros et al. [4] studied the mechanical integrity of CNT and ITO coated PET films under monotonic and cyclic uniaxial tension with in-situ electrical resistance monitoring. They observed that the critical strain for $25 \%$ electrical resistance increase is 10 times higher for CNT films than that observed for their ITO counterparts. Unlike the ITO brittle layer on 
compliant substrate, failure of the CNT layer was found to be controlled by catastrophic substrate failure.

\subsubsection{Bending and buckling experiments}

In a two-point bending test, a specimen is bent between two parallel plates. Electrical resistance measurements and optical images can be acquired, in-situ, to evaluate the degradation of the conductive coating (Fig. 2.5).

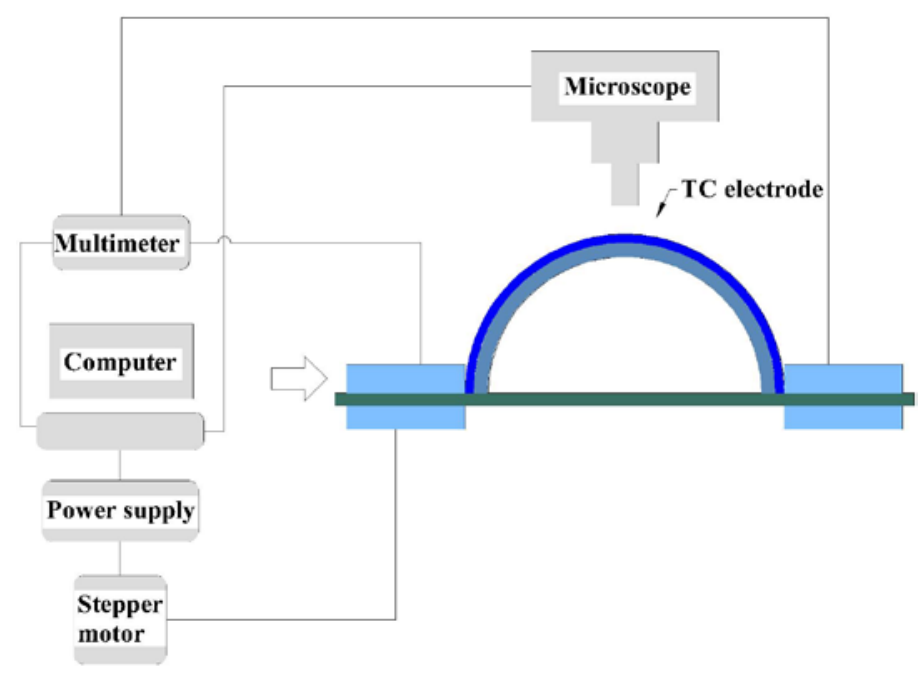

Fig. 2.5 Schematic of bending testing apparatus.

Chen et al. [74] used a controlled buckling test (i.e. two-point bending test with clamped ends) to investigate the fracture properties of ITO deposited on PET. The ITO surface was placed under both tension and compression. Electrical resistance measurements were monitored in-situ. They observed that the critical strain under compression loading for the ITO film was $1.7 \%$ 
while under tensile loading it was $1.1 \%$. Therefore, ITO surface under tension is more critical than when it is placed under compression. They also showed that, under tension, the failure mechanism is cracking of the film while in compression it is buckling delamination and cracking [75].

In addition, Abdallah et al. [76] investigated the buckle evolution of ITO layers, deposited on high temperature aromatic polyester substrates, using a mechanical bending device. In-situ optical microscopy of the layered structures under a uniaxial compressive strain was used to determine the buckle delamination rate at different applied strains. They observed an increase in the delamination rate by increasing the applied compressive strain. This increase was attributed to the increase in elastic energy applied to the crack tip. Thus, more elastic energy can lead to the creation of a new crack surface. In addition, edge defects were observed to affect not only the buckle initiation but also the buckle propagation. Increasing the number of edge defects at the specimen edge resulted in a decrease of the residual strains in the vicinity of the edge defect. As a consequence, less energy remains available for the delaminated buckle front to propagate further and therefore the buckle arrest [77].

Furthermore, Yang and Park [78] investigated the effect of inserting ductile metal interlayers, such as Ag, on both crack and buckling delamination resistance of ITO-coated polymeric substrates. They used a two-point bending device to measure the strains at crack initiation and delamination under tension and compression respectively. They found that, compared to the samples without interlayers, both the crack and delamination resistances of ITO are significantly improved by Ag interlayers over increased thickness. They observed that the cohesive and adhesive strength improvement was due to the increase in crystallinity of ITO by the Ag interlayer. This improved crystallinity of ITO due to Ag interlayer was observed by XRD. 
However, as thickness of the ductile interlayer increased, optical transparency was observed to decrease. Also, the internal stress relief exhibited by the addition of interlayers was found to have a negligible effect on crack resistance, as compared to crystallinity.

Moreover, Trottier et al. [79] studied the cyclic bending and monotonic tensile deformation of CNT films on PET substrates. It was reported that the maximum changes in electrical resistance under cyclic bending ( $0.7 \%$ strain and 2500 cycles) and monotonic tensile deformation ( $18 \%$ strain) were less than $0.5 \%$ and $14 \%$, respectively.

Finally, the buckle initiation and delamination of ITO lines on arylite substrate was investigated previously [77]. The total number of initiated buckles was found to increase with increasing compressive strain and/or loading time. Also, buckles were observed to initiate from etch defects and imperfections in the ITO layer. For example, the size of an initial defect able to initiate layer buckling was found to be approximately 15 times the layer thickness.

\subsubsection{Cyclic loading experiments}

Fatigue of flexible transparent electrodes is caused by cyclic or repeated loading. A thin film subjected to cyclic loading may fail due to progressive and localized damages even if the stresses and strains are below the electrode's yield strength. One advantage using fatigue characterization of TCO is that the ability to obtain cycles to failure data. This is very important in life prediction of such materials. There are limited previous fatigue studies on TCO.

Gorkhali et al. [80] used cyclic loading experiments (Fig. 2.6) to investigate the failure mechanisms of ITO-coated PET. An ITO-PET sheet was repeatedly rolled and unrolled around 
mandrels of varying diameters for a strain range between 0.6 and $2 \%$ while the resistance was monitored in-situ.

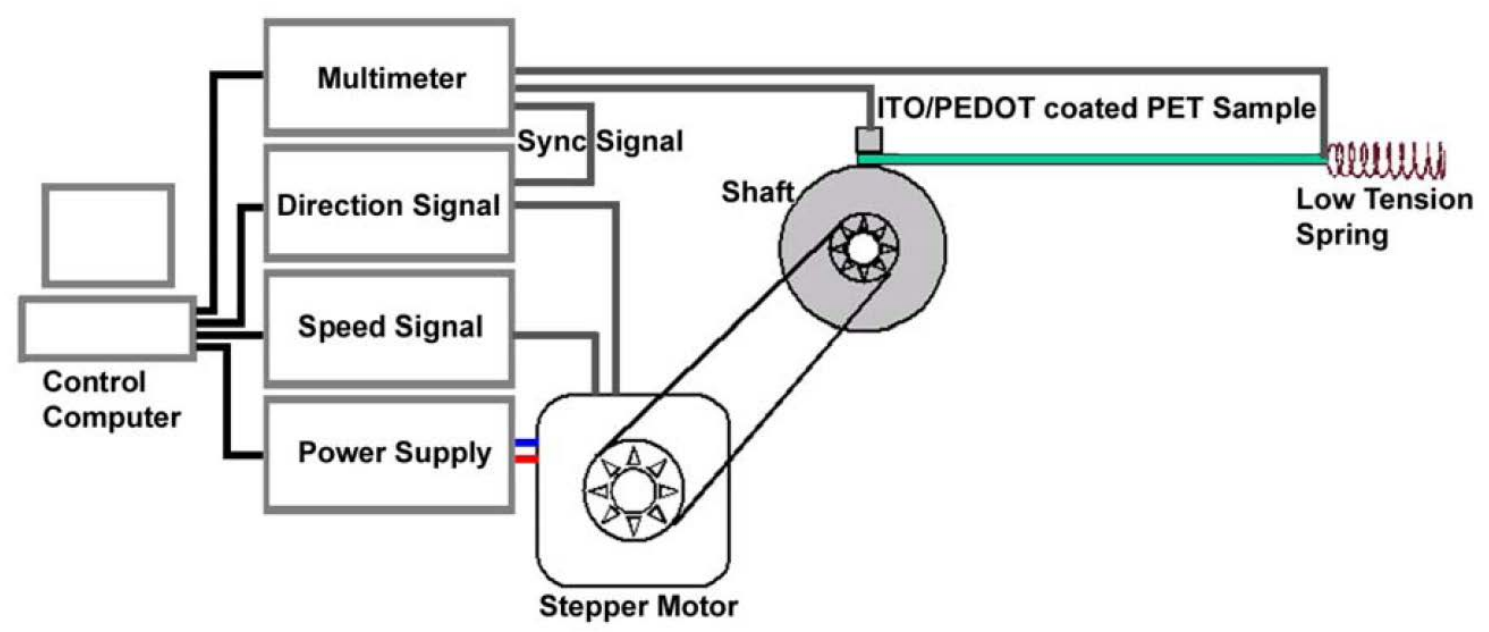

Fig. 2.6 Schematic of custom-built apparatus to monitor resistance during cyclic mandrel bending [80].

They observed three regimes of electrical resistance increase for the cyclic loading of ITO-coated PET as shown in Fig. 2.7. Between 50-100 cycles there was an increase in resistance due to sample dimension changes, as the PET reached dimensional equilibrium. Once the system stabilized, a slow increase in resistance up to approximately 50,000 cycles, which was found to be due to cracking of the ITO layer, was observed. Above 50,000 cycles severe cracking occurred and led to catastrophic failure of the ITO film. 


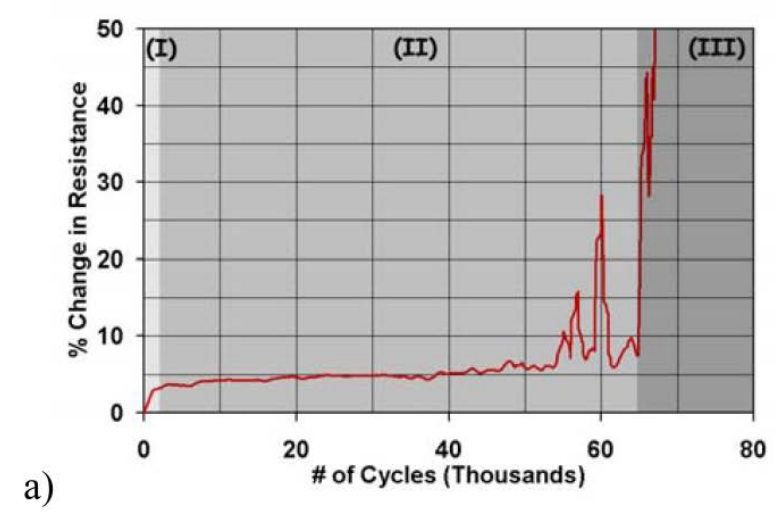

b)

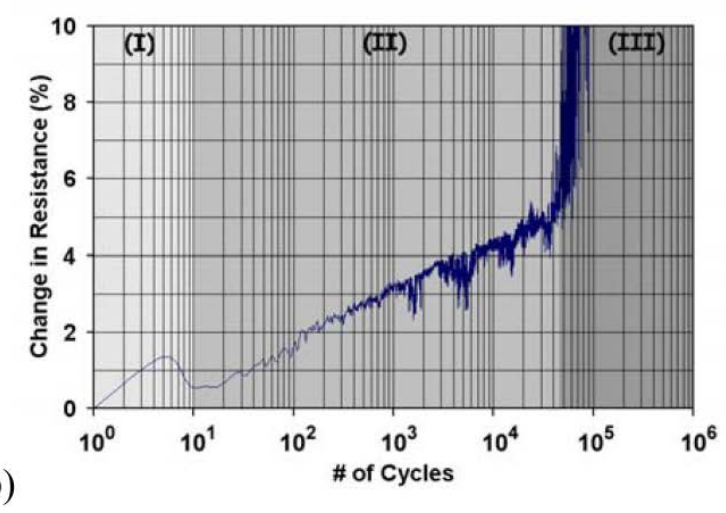

Fig. 2.7 Fatigue test until catastrophic failure of ITO samples. Linear scale (left) and logarithmic scale (right) [80].

Alzoubi et al. [81] investigated the high cycle bending fatigue of ITO-coated PET substrate in order to establish a base line for comprehensive reliability studies of ITO thin films on flexible substrates. The percent change in electrical resistance was measured at a specific number of cycles. It was found that the size of bending diameter and the number of bending cycles have a pronounced influence on the conductivity of the ITO layer. For example, at 200 cycles, the percent change in electrical resistance for samples bent around the $5.0 \mathrm{~mm}$ and 8.9 mm mandrel diameters were $860 \%$ and $640 \%$, respectively. However, at 500 cycles, the percent change in electrical resistances for samples bent around the same diameters were $6250 \%$ and 3360\%, respectively.

Also, Koniger and Munstedt [82] built a sophisticated device to investigate the electrical behavior of conductive layers on flexible substrates under oscillatory bending both in tension and under compression. For sputtered ITO coatings on PET substrates, a dramatic increase of the electrical resistance was observed for a bending radius smaller than $14 \mathrm{~mm}$ (strain $~ 0.6 \%$ ) due 
to cracks spanning the whole sample width. The higher the oscillatory bending amplitude, the more pronounced the increase of ITO electrical resistance.

Furthermore, Choa et al. [83] reported the influence of twisting loading on the mechanical integrity of indium zinc oxide (IZO) and IZO/Ag/IZO multilayer electrodes deposited on PET by continuous roll-to-roll sputtering. They used a lab-made twisting configuration (Fig. 2.8). Both crack density and electrical resistance increased with increasing twisting angle. They found that the critical twisting angle was $20^{\circ}$ for IZO and $26^{\circ}$ for IZO/Ag/IZO multilayer.

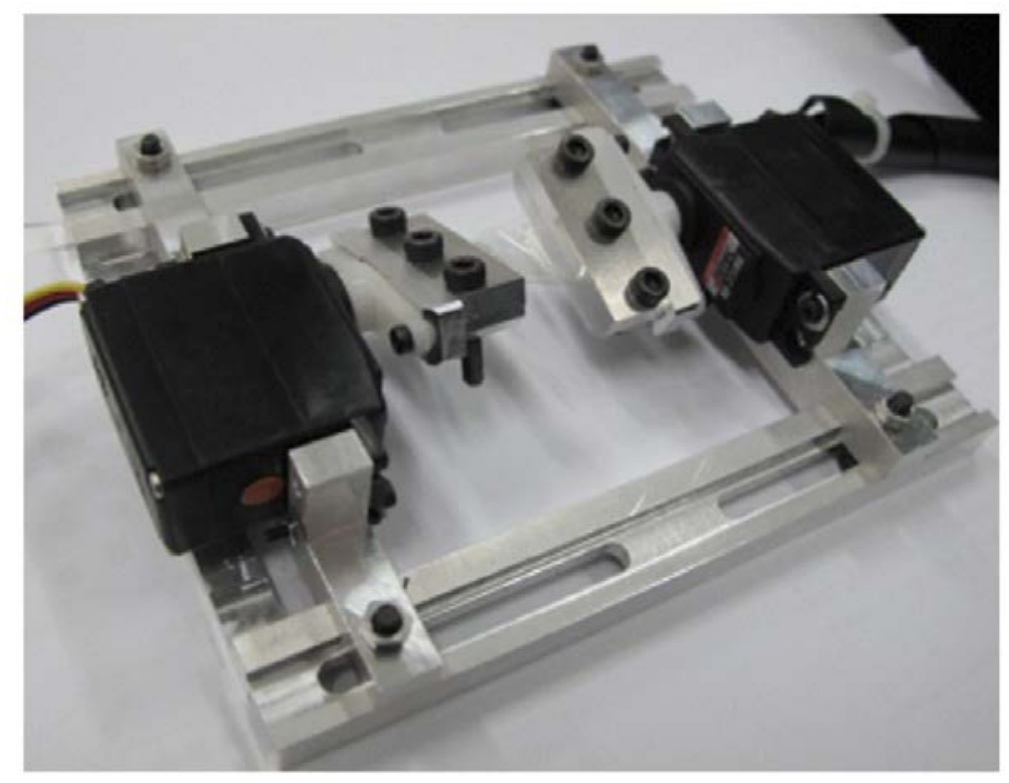

Fig. 2.8 Photograph of the lab-made twisting test apparatus [83].

Finally, Lan et al. [84] conducted static and dynamic bending tests in order to investigate the durability of ITO films deposited on PET by thermionic emission (TE) enhanced sputtering. They found that the critical radius of curvature was 13.9 and $17.0 \mathrm{~mm}$ for the ITO-coated PET 
with and without TE, respectively. The dynamic bending test showed that the ITO-coated PET with TE is found to be durable for 1200 cycles, while the corresponding number for the ITOcoated PET without TE is smaller than 50 cycles. They also observed that the ITO film deposited using TE exhibited a higher degree of crystallinity and film adhesion that deposited without TE. They concluded that TE improved the optical and electrical properties of the ITO coatings.

\subsubsection{Biaxial testing of flexible transparent electrodes}

Most of the researches previously reported on the reliability of TCO were performed on one-dimensional loadings. However, operations on thin films such as heating and cooling cycles during lamination and welding with other polymer films introduce almost always biaxial stress states [85]. This is because there is a thermal mismatch and lattice mismatch between brittle coatings and polymer substrates. Therefore, investigation of such films by means of biaxial loadings is important.

Leterrier et al. [85] studied the fragmentation process of 53 and $103 \mathrm{~nm}$ thick silicon oxide coatings on PET films under biaxial loading by means of a bulging cell mounted under an optical microscope. The examination of the fragmentation process of the coating under increasing pressure levels revealed that the crack onset strain of the oxide coating is similar to that measured under uniaxial tension. For example, the crack onset strain under biaxial loading was $1.8 \%$ and $1.5 \%$ for the 53 and the $103 \mathrm{~nm}$ thick coatings, respectively. The fragmentation of the coating under biaxial tension was also characterized by complex dynamic phenomena resulted in considerable broadening of the fragment size distribution. 
In addition, Andersons et al. [86] investigated crack patterns of $100 \mathrm{~nm}$ thick silicon oxide coatings on PET, polypropylene (PP), and polyamide (PA) substrates under biaxial loading. A bulging cell with a stepwise pressurization system was mounted under an optical microscope. Fragment areas were found to follow the exponential distribution at low strains close to the COS. With the increase of biaxial strain, change of the fragment area distribution was observed due to stress transfer and crack propagation. In silicon oxide on PP and PA, film fragmentation proceeded in a binary manner, i.e. coating fragment breakup due to crack propagation always lead to generation of two new fragments. By contrast, in silicon oxide on PET, sequential crack branching was observed with the increase of strain as an additional failure process to binary cracking.

\subsubsection{Wear, nanoscratch and nanoindentation experiments}

In addition to uniaxial and biaxial stress failure mechanisms, flexible transparent electrodes may be subjected to wear during processing and operation.

Cairns et al. [16] studied the wear damage of ITO coated PET substrate with a pen stroke applied repeatedly on the PET surface. The schematic of the system is shown in Fig. 2.9. They observed adhesive wear in the form of ITO flakes transferred from the ITO on PET substrate to the ITO on glass substrate. Pitting of polymer was evident between 5,000 and 10,000 cycles. After 40,000 cycles a large amount of polymer was pulled through the ITO pits. Also, cracking was observed at 50,000 cycles. 


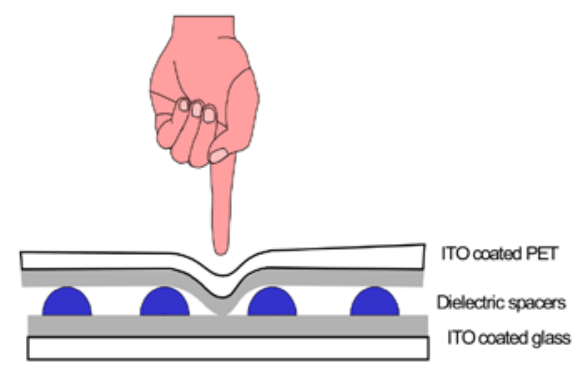

Fig. 2.9 Schematic diagram of the operation of a resistive touch screen [16].

In addition, Kim et al. [87] investigated the adhesive and wear properties of ITO thin films on glass substrate using scratch and wear tests. In the scratch test, a micro-blade was drawn over the films while in the wear test, sapphire, SS (stainless steel), and WC (tungsten carbide) balls slid repeatedly on the films with a normally applied constant load. The electrical resistance of the ITO surface was monitored in-situ and it was used to measure both the critical load to completely cut through the film and the critical number of sliding to completely wear the ITO film. The critical numbers of sliding for wear testing using sapphire, SS, and WC balls were found to be 7111, 1262, and 5799, respectively. In addition, the critical load of ITO film under scratch test was estimated to be $0.54 \mathrm{~N}$.

Furthermore, Sierros et al. [88] studied the wear of an ITO flat surface against an ITO flat counterface using a custom-built reciprocating wear tester at a normal load of $3.5 \mathrm{~N}$ and up to 12,240 cycles. In-situ electrical resistance measurements indicated an abrupt increase above 10,000 reciprocating cycles. Weight loss in the case of the bottom counterface sample initially measured to be around $0.1 \mathrm{mg}$ and increased up to $1 \mathrm{mg}$ with increasing number of reciprocating cycles. They proposed that the wear mechanisms of ITO surfaces include cohesive failure within 
the film, adhesive wear between the ITO film and PET substrate, plastic deformation of the PET substrate and abrasive wear of the ITO film.

Moreover, Tseng [89] used a patterning technique that utilizes an AFM tip as a scratch tool, also known as AFM scratching, to study the scratch characteristics of silicon. He experimentally studied and mathematically correlated the scratch parameters, including the applied tip force and the number of scratches, on the size of the scratched geometry. The dimensions of the grooves scratched were related to the normal force in a logarithmic form, and with the scratch cycle number in a power law form. He compared hardness, wear coefficient, and scratch ratio ( ratio of the scratch penetration depth to the threshold force) in order to assess the scratchability property. Scratch ratio was found to be an appropriate indicator for measuring the degree of the ease or difficulty of a material scratched by an AFM tip.

Also, Sierros et al. [90] investigated the effect of normal and tangential spherical loading on the mechanical durability of ITO-coated PET film using a spherical nanoindenter and scratch tester with built-in optical microscopy capability. They observed that crack initiation at $40 \mathrm{mN}$ normal load or 534 MPa mean contact pressure. At higher loads, brittle ring cracking was observed and when loading increased to $200 \mathrm{mN}$, secondary cracks were formed. They also found that the indent imprint radius on the ITO surface increased linearly with increasing load. It was found that increased thickness led to larger crack spacing. During scratch testing, cracking and buckling spallation of the coating were observed. Both failure mechanisms exhibited a thickness dependence.

Finally, Hecht et al. [91] conducted single-point stylus-pen actuation tests on CNT coated PET films for resistive touch screens. They reported that no failure is observed up to 3 million actuations. 


\subsubsection{Electromechanical and corrosion experiments}

Flexible transparent electrodes degrade when subjected to tensile, buckling, bending, wear, and cyclic loadings, but the presence of corrosive agents may aggravate this behavior either by increasing the rate of crack growth and rate of wear, or decreasing the mechanical load required to initiate cracks.

\subsubsection{Corrosion of flexible transparent electrodes}

Degradation of transparent electrodes with acid containing adhesives which are commonly used in optoelectronic device manufacturing may lead to reduction in optical transmittance, high sheet resistance, and substantial changes in surface morphology and composition. In most adhesive polymers a typical concentration of acrylic acid in the range of 0.05-0.3 $\mathrm{M}$ is grafted to improve their properties [92].

Folcher et al. [93] studied the electrochemical stability of ITO films in different concentrations of $\mathrm{HCl}$. The corrosion behavior of the films was investigated using in-situ mass measurements and direct imaging of surface morphology. It was found that radical species such as $\mathrm{Cl}^{0}$ and $\mathrm{OH}^{0}$ were created during positive potential corrosion conditions and attacked the indium-oxygen surface bonds. TEM images showed a preferential dissolution at grain boundaries of the polycrystalline ITO films. In addition, the dissolution rate increased significantly when the $\mathrm{pH}$ was decreased indicating that the anodic dissolution of ITO in $\mathrm{HCl}$ solution is not a pure electrochemical process but it involves a chemical contribution such as oxygen evolution pushing away the $\mathrm{Cl}^{0}$ radicals. 
Monk and Man [94] investigated the etching behavior of ITO in varying concentrations of aqueous hydrochloric acid in the range of 1 to $10^{-3} \mathrm{~mol} . \mathrm{dm}^{-3}$. They found that, during etching, $\mathrm{In}^{3+}$ reduced to metallic indium and the surface of the ITO film became severely rough.

\subsubsection{Combined stress and corrosion testing}

In addition to degradation due to corrosion, transparent electrodes may fail under the combined effect of stress and corrosion. Stress corrosion cracking (SCC) occurs when mechanical strain is applied to a specimen in the presence of a corroding environment. In such a situation both effects (i.e., stress and corrosion) are likely to aggravate and complement each other. SCC is also a local phenomenon, and a relatively large region of the material surface may remain undamaged. As a crack propagates a crevice may appear, in which the $\mathrm{pH}$ of the acid steadily rises as corrosion occurs, due to a lack of mixing with more dilute acid from an area outside the crevice [95]. This can lead to high acid concentrations in the crevice as well as high stress concentrations at the crack tip, which may further enhance the severity of the cracking process. In ITO thin films, the cause of SCC failure is the presence of a network of a large number of small cracks rather than one critical crack [71], but localized acid concentrations and stress concentrations may still play a large role in the failure process.

Ramji et al. [96] reported that cracks in patterned ITO-PET sheets initiate from microcracks caused by the patterning process. It was found that the rate of crack growth in ITO film is accelerated by the combined effect of corrosion due to acrylic acid from pressure sensitive adhesives and the applied stress. 
Also, Sierros et al. [97] investigated the combined effect of applied strain and acrylic acid (0.1 - 0.9 M concentration) on transparent conductive layers of ITO sputtered PET substrate. It was found that the presence of acid can cause cracks to initiate at strains as low as a quarter of those observed for films with no corrosion, indicating that SCC is a significant process. In addition, corrosion was most prevalent at regions of high surface roughness, where a larger surface area exposed to the acid, and the presence of crevices for increased acid concentration are most likely.

Finally, Hamasha et al. [98] investigated the effect of temperature, humidity, bending fatigue and interaction among them on the percent change in electrical resistance of sputtered ITO coated PET substrates. Two sets of experiments were conducted on the ITO film. The first set of experiments was conducted on samples under different temperature $\left(20\right.$ and $\left.70^{\circ} \mathrm{C}\right)$ and humidity (20 and 80\%) combinations while subjected to bending fatigue. The other set of experiments was conducted on samples with the same combinations of temperature and humidity but without fatigue. The change in electrical resistance of the specimens under the combinations of high temperature and high humidity with cyclic bending load was found to be $17 \%$. On the other hand, specimens without fatigue loading showed a $4 \%$ change in electrical resistance. They suggested that cyclic bending with high temperature and humidity of ITO films should be avoided during both manufacturing and applications.

\subsubsection{Tribo-corrosion behavior of transparent electrodes}


Investigation of the tribo-corrosion behavior of the electrodes is critical in the design and manufacturing of reliable flexible devices which will remain functional after repetitive mechanical flexing under harsh environmental conditions.

The behavior of dry and wet reciprocating sliding of flat ITO against another flat ITO surface in acidic solution has been studied previously [90]. The corrosive action of the acidic solution on the ITO degradation was observed to be a secondary effect. The primary effect was the tribological interaction of the two surfaces that led to wear and loss of functionality. A particular advantage when conducting tribological tests on such structures is that one can monitor the electrical resistance in-situ and correlate changes of the ITO microstructure with electrical resistance changes [87, 90]. 


\section{MATERIALS AND EXPERIMENTAL METHODS}

\subsection{Materials}

In this project ITO and CNT conducing layers on polymer substrates were used. Also, patterned ITO layer and multilayers of ITO with metallic alloys were utilized. This chapter describes the materials used according to the various experimental methods employed.

\subsubsection{Corrosion of ITO films}

ITO film (CPFilms Inc., USA) deposited at low temperature on a PET polymer substrate by DC magnetron sputtering was used as the working electrode. The thickness of the ITO layer is $70 \mathrm{~nm}$ with a sheet resistance of $100 \Omega /$ sq. The ITO film is composed of $90 \mathrm{wt} . \% \mathrm{In}_{2} \mathrm{O}_{3}$ and 10 wt. \% $\mathrm{SnO}_{2}$. The ITO samples were cleaned with distilled water and dried with cold air before performing the electrochemical measurements using acrylic acid (Sigma-Aldrich) as an electrolyte.

\subsubsection{Mechano-chemical experiments}

Another set of ITO films (CPFilms Inc., USA) deposited at room temperature on PET substrates $(180 \mu \mathrm{m}$ thick) by DC magnetron sputtering (sputtering power $\approx 1 \mathrm{KW}$ ) were used in the mechano-chemical experiments. During sputtering, the processing gas was argon with a partial pressure of approximately 0.5 Pa whereas oxygen was used as a reactive gas with a partial 
pressure of approximately $2 \mathrm{MPa}$. The deposition rate of the ITO film was approximately 5 $\mathrm{nm} / \mathrm{min}$. The deposited films, as measured by a stylus profilometer (Veeco Dektak 150), were 70 and $200 \mathrm{~nm}$ thick with sheet resistances of 100 and $70 \Omega /$ sq, respectively. The ITO target was composed of 90 wt. $\% \mathrm{In}_{2} \mathrm{O}_{3}$ and 10 wt. \% $\mathrm{SnO}_{2}$. Sheet resistances were measured using a fourpoint probe system (Electronic Design to Market, USA).

Also, CNT films (Unidym, USA) on PET substrates (180 $\mu \mathrm{m}$ thick) were used for comparison purpose. The carbon nanotubes were grown via chemical vapor deposition and subsequently purified via air oxidation followed by acid washing to remove residual metal catalyst and amorphous carbon. The resulting CNTs consisted of largely single-walled and double-walled tubes. The CNTs were dispersed in a $1 \%$ aqueous solution of sodium dodecyl sulfate surfactant at $0.01 \mathrm{wt} \%$ and spray coated onto the PET sheet which was heated to $100{ }^{\circ} \mathrm{C}$. The films were then rinsed in de-ionized water to remove residual surfactant. The resulting dry CNT film is approximately $15 \mathrm{~nm}$ thick as measured using atomic force microscopy. A polymethyl methacrylate (PMMA) layer was coated with a Mayer rod from a $0.5 \mathrm{wt} \%$ solution of PMMA in methyl ethyl ketone over the dry CNT film to enhance film adhesion to the PET and provide enhanced chemical/environmental resistance. The dry thickness of the binder layer is $\sim 50 \mathrm{~nm}$, thin enough such that it does not electrically insulate the surface of the CNT film. The polymer coating is not continuous and in some regions the CNT layer is exposed allowing a conducting path. The sheet resistance of the CNT film was approximately $350 \Omega / s q$.

\subsubsection{Controlled buckling experiments}


A $100 \Omega / s q$ ITO film surface was cleaned using isopropyl alcohol and DI water and then dried using nitrogen in order to be ready for patterning of different shapes and sizes (Fig. 3.1a) using photolithography for controlled-buckling experiments. The specimen surface was spincoated (2000 rpm, 1min) with an AZ 3330 photo-resist and then it was pre-baked at $90{ }^{\circ} \mathrm{C}$ for 1 min. After exposure through a photo-mask and post-bake at $90{ }^{\circ} \mathrm{C}$, the resist was developed in an AZ 3330 solution. The specimen was then etched in $2 \%$ oxalic acid $\left(\mathrm{C}_{2} \mathrm{H}_{2} \mathrm{O}_{4}\right)$ at an approximate etch rate of $10 \mathrm{~nm} / \mathrm{min}$. Finally, the remaining photo-resist was removed using acetone, and followed by both cleaning in DI water and nitrogen blowing.

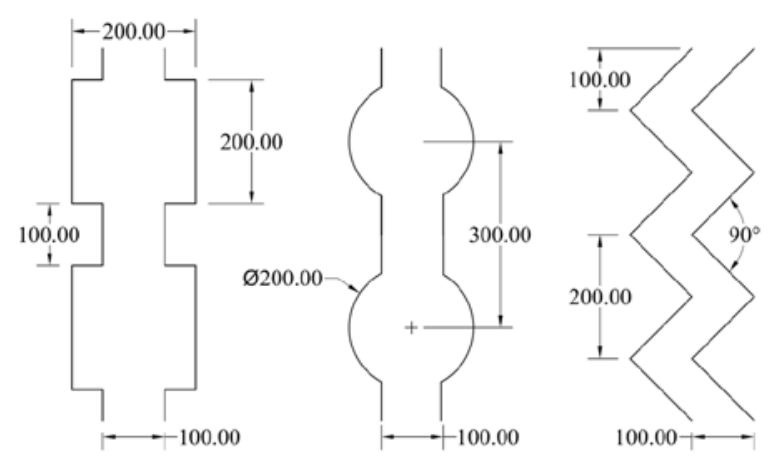

(a)
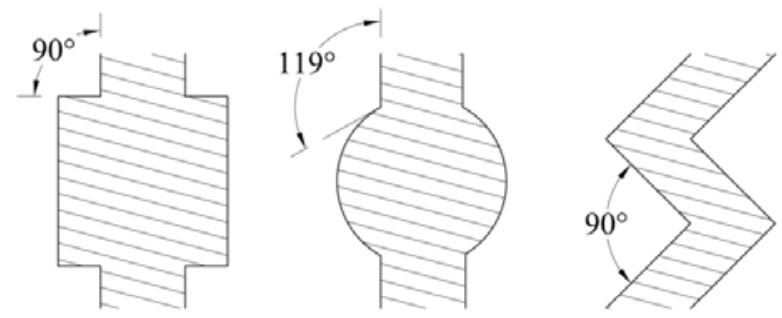

(b)

Fig. 3.1 Different patterns of ITO coated PET films with (a) smaller size dimensions and (b) sharpness of corners. All dimensions are in $\mu \mathrm{m}$. 


\subsubsection{Tribo-corrosion experiments}

A Pure Ag, Ag-Au (2 wt\% Au) and Ag-Pd (3 wt\% Pd) alloy layer ( 10 nm thick) was sputtered between two ITO layers of $40 \mathrm{~nm}$ thickness each (Fig. 3.2). The bottom ITO layer was sputtered at room temperature on a $100 \mu \mathrm{m}$ thick polyethylene terephthalate (PET) substrate. The top ITO surface was sputtered, at room temperature, on top of the Ag/Ag-alloy surface and exhibits a root mean square roughness of approximately $6 \mathrm{~nm}$ as measured by atomic force microscopy in contact mode. The sputtering power was approximately $1 \mathrm{~kW}$ and Ar was used as the processing gas. Therefore, a multilayer structure was deposited by sputtering successive layers of ITO - Ag/alloy - ITO layers on PET substrates. Polytetrafluoroethylene (PTFE) balls, $10 \mathrm{~mm}$ in diameter, were used as the counter body. Tests done on ITO-Ag-ITO and ITOAg/alloy-ITO flat films using a PTFE ball counterface in a reciprocating fashion are summarized in Table 3.1. During testing $\mathrm{NaCl}$ solution (1 M) was used as the corrosive medium.

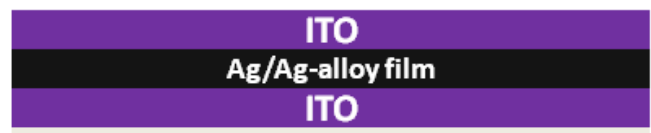

Polymer substrate

Fig. 3.2 Cross-sectional view of the sample under tribo-corrosion investigation. 
Table 3.1 Materials used in tribo-corrosion experiments.

\begin{tabular}{cccccc}
\hline $\begin{array}{c}\text { Base } \\
\text { substrate }\end{array}$ & $\begin{array}{c}\text { Bottom } \\
\text { layer }\end{array}$ & Interlayer & $\begin{array}{c}\text { Top } \\
\text { layer }\end{array}$ & $\begin{array}{c}\text { Total thickness } \\
(\mathrm{nm})\end{array}$ & Counterface \\
\hline \hline PET & ITO & $\mathrm{Ag}$ & ITO & 90 & PTFE \\
PET & ITO & $\mathrm{Ag} \mathrm{3} \mathrm{wt}^{2} \mathrm{Pd}$ & ITO & 90 & PTFE \\
PET & ITO & $\mathrm{Ag} 2 \mathrm{wt} \% \mathrm{Au}$ & ITO & 90 & PTFE \\
\hline
\end{tabular}

\subsection{Experimental methods}

In order to meet the objective of this research, electrochemical, electromechanical, and mechanochemical experimental procedures are performed using new and existing testing methods.

\subsubsection{In-situ electrical measurements for corrosion experiments}

ITO samples were immersed in acrylic acid concentrations ranging from 0.05 to $0.3 \mathrm{M}$ and the change in electrical resistance was monitored in-situ using an Agilent 34970A data acquisition/switch unit in order to optimize the critical acid concentration.

\subsubsection{Electrochemical impedance spectroscopy}

Electrochemical measurements were performed for the ITO film, to understand the change in the properties of the film with exposure time to acrylic containing adhesives. The electrochemical measurements were carried out using a Potentiostat/Galvanostat (Solartron 
Analytical, 1287) controlled by CorrPlot software version 2.9C (Scribner Associates, Inc., UK) and a flat cell (EG \& G Model K0235). Platinum gauze was used as a counter electrode and saturated $\mathrm{Ag} / \mathrm{AgCl}$ electrode was employed as a reference electrode. The ITO film, with an exposed area of $1 \mathrm{~cm}^{2}$, was used as the working electrode. The open-circuit potential will be measured until dynamic stability is achieved between the working TC electrode and acrylic acid electrolyte. Before each potentiodynamic polarization and EIS experiments, the electrode was allowed to corrode freely and its open circuit potential was recorded as a function of time up to 60 min. The potentiodynamic polarization measurement was performed by scanning the potential starting from $-250 \mathrm{mV}$ to $1600 \mathrm{mV}$ with respect to open-circuit potential at a scan rate of 1 $\mathrm{mV} / \mathrm{sec}$. The potentiodynamic polarization data was analyzed using CorrVeiw software. The EIS measurements were performed using an impedance analyzer (Solartron Analytical, 1255B) connected with a potentiostat (Solartron Analytical, 1287). The EIS spectrum was recorded over a frequency range of $1 \mathrm{MHz}$ to $10 \mathrm{mHz}$. The applied bias voltage and $\mathrm{AC}$ amplitude were set at open-circuit potential of the electrode and $10 \mathrm{mV}$, respectively. The EIS data was plotted using ZPlot and the curve fitting of EIS data to an electrical equivalent circuit was performed using the ZView software version 2.9C (Scribner Associates, Inc., UK). All the electrochemical experiments were performed three times to ensure reproducibility of the results.

\subsubsection{Mechano-chemical testing}

During corrosion testing, non-strained flat л-shaped samples were immersed in a $0.05 \mathrm{M}$ acrylic acid solution, and the change in electrical resistance was monitored in-situ. Bending, 
fatigue, bending-corrosion, and fatigue-corrosion characterization was conducted by means of a custom-built cyclic uni-axial loading apparatus (Fig. 3.3).
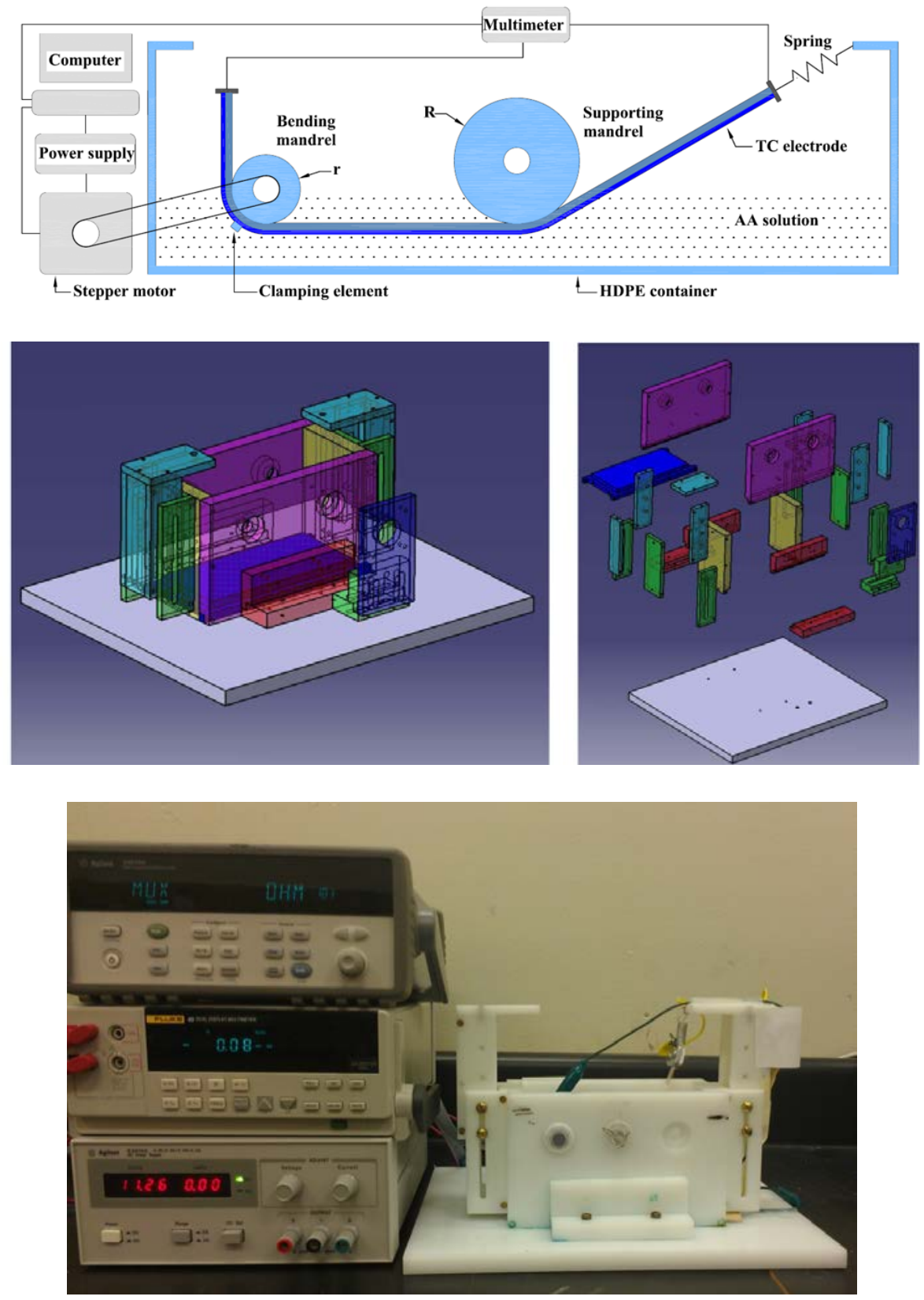

Fig. 3.3 Schematic, 3D and exploded CATIA model (Dassault Systemes), and picture of the custom-built bending cyclic loading apparatus. 
In this set-up, one end of the specimen is fixed to a mandrel made from chlorinated polyvinyl chloride (c-PVC) using a high-density polyethylene (HDPE) plate whereas the other end is clamped between a pair of HDPE plates which were connected to a low tension spring ( $\mathrm{k}$ $=68.4 \mathrm{~N} / \mathrm{m}$ ). A strip of copper foil is used for electrical contact. A relatively large c-PVC mandrel ( $\mathrm{R}>>\mathrm{r}$ ) supported the specimen in a horizontal position and prevents it from sagging. A stepper motor (MDrive M-17, IMS) is used to rotate the mandrel at a frequency of 2 cycles/s. During both static bending-corrosion and fatigue-corrosion testing the HDPE container is filled with acidic solution. The change in electrical resistance is monitored in-situ using an Agilent 34970A data acquisition/switch unit. Specimens were cut into strips of $10 \mathrm{~mm}$ in width and 188 - $200 \mathrm{~mm}$ in length in accordance with the bending diameter used. The diameter of mandrel, specimen length, and applied strain are listed in Table 3.2. The approximate bending strain using such mandrels is calculated using the following equation, Eq. 3.1 [74].

$$
\varepsilon=\frac{t_{s}+t_{f}}{2 R}
$$

where $t_{s}$ and $t_{f}$ are the thickness of the substrate and film, respectively.

Table 3.2 Size of mandrel, specimen length, and approximate applied strain.

\begin{tabular}{ccc}
\hline $\begin{array}{c}\text { Mandrel diameter } \\
(\mathrm{mm})\end{array}$ & $\begin{array}{c}\text { Specimen length } \\
(\mathrm{mm})\end{array}$ & $\begin{array}{c}\text { Applied strain } \\
(\%)\end{array}$ \\
\hline \hline 17.78 & 184 & 1.00 \\
22.55 & 190 & 0.80 \\
29.10 & 200 & 0.60 \\
\hline
\end{tabular}




\subsubsection{Controlled-buckling testing}

Controlled buckling characterization of patterned ITO films was conducted by means of a manually operated custom-built apparatus (Fig. 3.4).

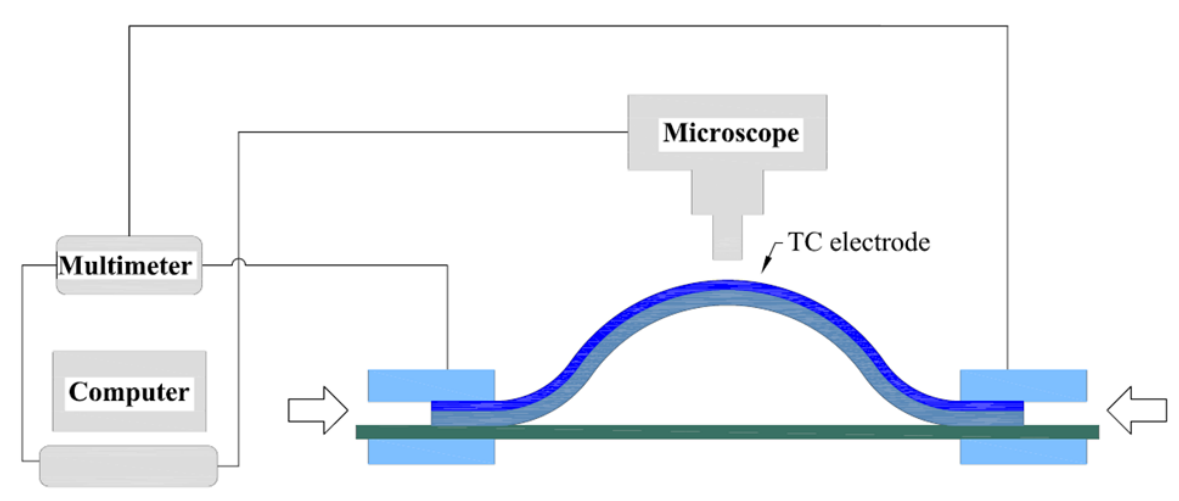

Fig. 3.4 Schematic of the custom-built manually operated buckling apparatus.

In this setup, the two ends of the specimen are firmly clamped on the buckling apparatus. The buckling apparatus was placed underneath an optical microscope (Leica) which is equipped with a frame grabber (Guppy, Allied Visions Technology). Sample displacement measurements were taken using a side-view digital imaging system (Celestron Digital Microscope) and they were analyzed using image analysis software (Image J. NIH USA). The buckling radius from the testing scheme (Fig. 3.5) was calculated using Eq. 3.2 [74].

$$
\frac{l}{R}=4 \mathrm{~K}(\mathrm{k}) \mathrm{k}
$$


where $K(k)$ is complete elliptic integral of the first, $k=\sin (\theta / 2), 1=L / 2$ for built-in ends. $K(k)$ is calculated using MatLab (MathWorks).

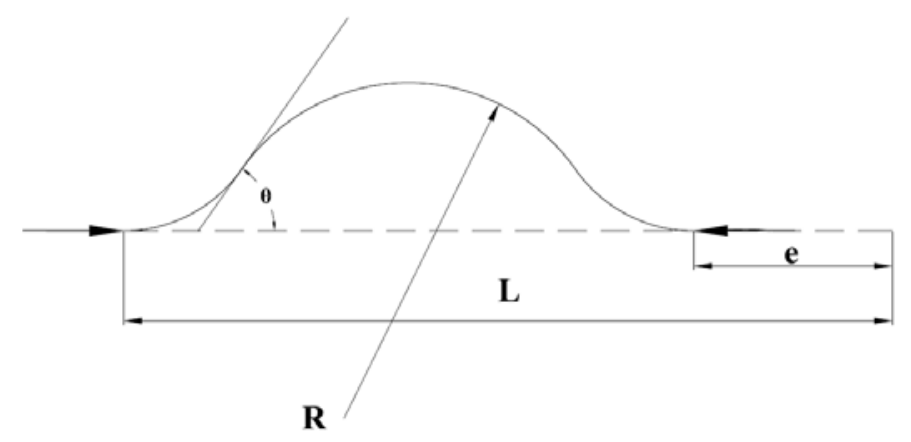

Fig. 3.5 Buckling test with built-in ends [74].

The resulting strain was calculated using Eq. 3.1. In-situ optical microscopy images were taken at small increments of applied strain in order to determine both tensile and compressive crack onset strains and to monitor the crack propagation. In addition, in-situ electrical resistance changes were monitored using a Fluke 45 dual display multimeter.

\subsubsection{Tribocorrosion testing}

The tribo-corrosion test was carried out in order to assess the combined wear and corrosion degradation of the TC electrodes. Testing was conducted by means of a custom-built reciprocating wear tester (Fig. 3.6). 


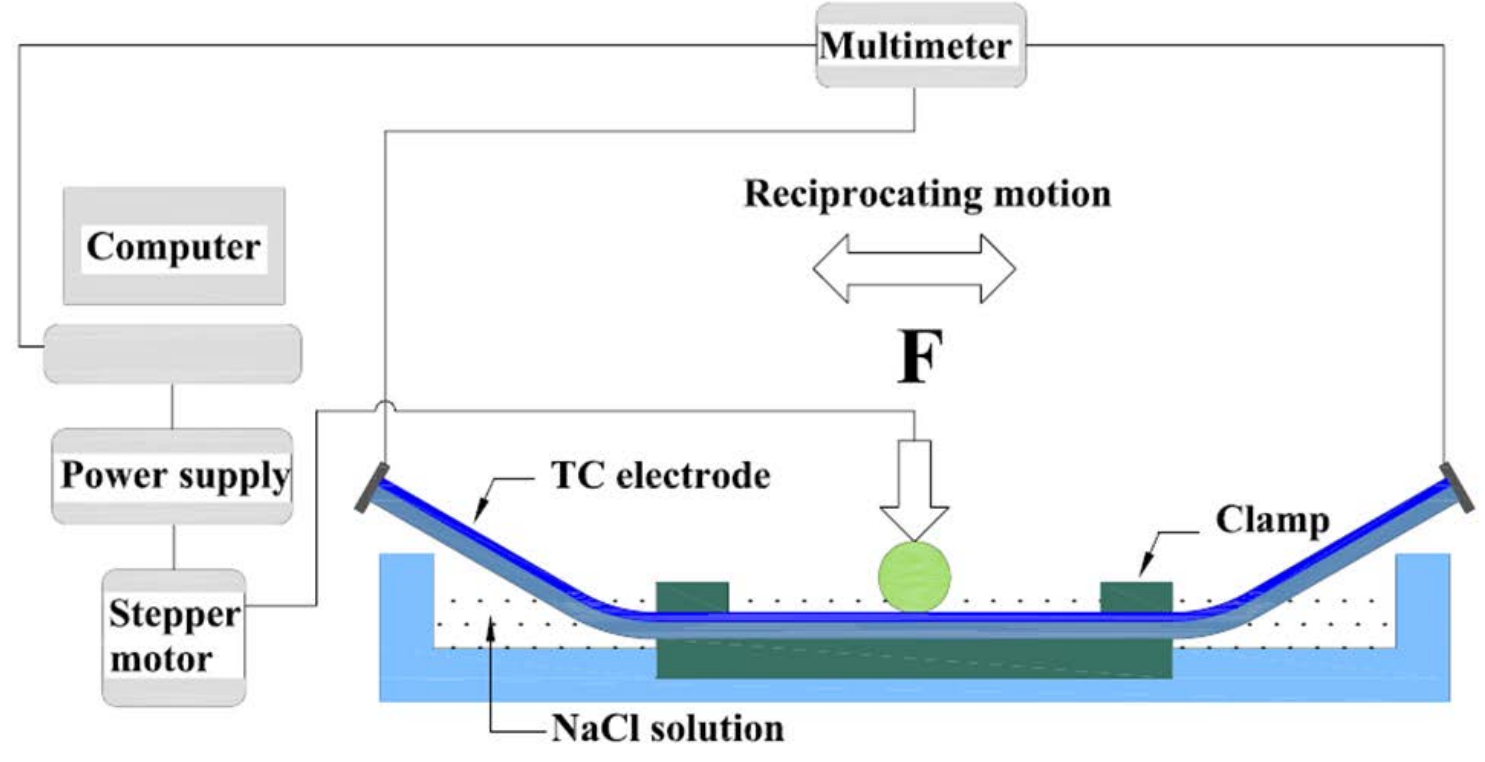

Fig. 3.6 Schematic of the custom-built reciprocating wear tester apparatus.

In this setup, the bottom counter face sample is clamped to an acrylic base. Particular care was taken when clamping the sample to the base in order to avoid stressing the sample. The sample was fitted inside a plastic container. Under corrosive sliding conditions the container was filled with $1 \mathrm{M}$ sodium chloride solution. A stepper motor (NM34A200, Zaber) controls the reciprocating end effector with stroke length $\mathrm{L}=50.8 \mathrm{~mm}$. The end effector is a $10 \mathrm{~mm}$ diameter polytetrafluoroethylene (PTFE) ball mounted on a c-PVC rod and was allowed to float freely in the vertical direction. PTFE was chosen because of its low friction coefficient and its corrosion resistance. The ball surface was replaced after the completion of each test and a fresh PTFE surface was used for the next test. A constant normal load $\mathrm{F}=3 \mathrm{~N}$ was applied. This load introduced a mean Hertzian stress equal to $12.02 \mathrm{MPa}$. Around $12.02 \mathrm{MPa}$ is a typical contact pressure under moderate handling and/or in-service conditions of solar energy components and many optoelectronic applications. The change in electrical resistance was monitored in-situ using 
an Agilent 34970A data acquisition/switch unit. Each test was conducted using a fixed reciprocating frequency of 3 cycles/min. Tests were run for up to a few thousand reciprocating cycles. In addition, weight measurements were conducted before and after each test for both the PTFE ball and TC electrode using a digital balance (DV215CD, Ohaus, $0.01 \pm 0.02 \mathrm{mg}$ resolution). After testing and before weighing the samples were washed in distilled water and dried using compressed air.

\subsubsection{Surface characterization}

- Stylus profilometry (Veeco Dektak 150) was used to measure film thickness and study its topography.

- Specimen surfaces were studied, after testing, using a Leica optical microscope equipped with a frame grabber (Guppy, Allied Visions Technology) at magnifications of 10, 20, $50 \mathrm{x}$.

- The surface morphology of the electrodes before and after testing was studied using a JEOL JSM - 7600F scanning electron microscopy (SEM) with a field emission gun. Before SEM analysis, the specimens were sputter coated with an approximately $10 \mathrm{~nm}$ thick Au layer using a Cressington 108 sputter coater.

- X-ray photoelectron spectroscopy (XPS) before and after testing was conducted using a Physical Electronics PHI 5000 VersaProbe system to analyze chemical composition through depth profiling. The X-ray beam was $100 \mu \mathrm{m}, 25 \mathrm{~W}$, and $15 \mathrm{KV}$, from a monochromatic Al Ka source using charge neutralization and pass energy of $29.35 \mathrm{eV}$.

\subsection{Design of experiments}


Design of experiment (DOE) was used to further investigate the effect of various design, process, and environmental factors on the functionality of ITO films under mechano-chemical testing. Four study factors were considered while change in electrical resistance was selected as a response. The study factors were: acrylic acid concentration, applied strain, film thickness, and number of cycles (NOC) for fatigue and combined fatigue-corrosion experiments. In addition, for bending and bending-corrosion time was considered as a study factor due to the static nature of these experiments. The levels of each factor chosen are presented in Tables 3.3 and 3.4, respectively. Furthermore, regression models were developed to predict the significant effects and synergies of the factors on the change in electrical resistance of the electrodes.

Table 3.3 Factors and levels for DOE analysis of fatigue and fatigue-corrosion experiments.

\begin{tabular}{ccccc}
\hline \multirow{2}{*}{ Factors } & \multicolumn{5}{c}{ Levels } \\
\cline { 2 - 5 } & 1 & 2 & 3 & 4 \\
\hline \hline Acid concentration (M) & 0 & 0.05 & - & - \\
Strain (\%) & 0.6 & 0.8 & 1.0 & - \\
Thickness (nm) & 70 & 200 & - & - \\
Number of cycles & 0 & 50040 & 100020 & 150000 \\
\hline
\end{tabular}

Table 3.4 Factors and levels for DOE analysis of bending and bending-corrosion experiments.

\begin{tabular}{ccccc}
\hline \multirow{2}{*}{ Factors } & \multicolumn{4}{c}{ Levels } \\
\cline { 2 - 5 } & 1 & 2 & 3 & 4 \\
\hline \hline Acid concentration (M) & 0 & 0.05 & - & - \\
Strain (\%) & 0.6 & 0.8 & 1.0 & - \\
Thickness (nm) & 70 & 200 & - & - \\
Time (s) & 0 & 25020 & 50010 & 75000 \\
\hline
\end{tabular}


Also, DOE with two replicates was used to further investigate the effects of the two factors - size and shape - on the change in electrical resistance at crack initiation observed using optical microscopy for patterned ITO films under controlled-buckling. The levels of both factors are shown in Table 3.5.

Table 3.5 Factors and levels for DOE analysis of controlled-buckling experiments.

\begin{tabular}{cccc}
\hline \multirow{2}{*}{ Factors } & \multicolumn{3}{c}{ Levels } \\
\cline { 2 - 4 } & 1 & 2 & 3 \\
\hline Size & Small & Large & - \\
Shape & Square & Zigzag & Circle \\
\hline
\end{tabular}

\subsection{Finite element analysis}

Finite element analysis (FEA) was performed using an engineering simulation software (ANSYS, Inc.) in order to predict the stress-strain distributions and potential crack initiation on the oxide coatings on polymer substrates under different deformation modes. A two-dimensional model was built using shell elements - SHELL91 as reported in previous studies [81, 99, 100]. The mechanical properties of the layered structure are shown in Table 3.6.

Table 3.6 Mechanical properties of materials for FEA [101, 102]

\begin{tabular}{ccc}
\hline Material & Young's modulus $(\mathrm{GPa})$ & Poisson's ratio \\
\hline \hline ITO & 119 & 0.34 \\
PET & 5.3 & 0.35 \\
\hline
\end{tabular}


In order to obtain an accurate model for bending experiments, a part of the sample length which is in contact with the mandrel circumference was considered. The length of the sample is calculated:

$$
l=\frac{\theta}{36} \pi d
$$

where $\mathrm{d}$ is the diameter of the mandrel and $\theta$ is the angle rotation of the mandrel in half cycle. A vertical displacement constraint was applied to all nodes at both edges of the film $\left(\mathrm{u}_{\mathrm{z}}=0\right)$. In addition, a vertical displacement constraint was applied to all nodes at the mid-section with a value $\left(\mathrm{u}_{\mathrm{z}}=\mathrm{h}\right)$ obtained from using the following equation.

$$
h=\frac{d}{2}(1-\sin \theta)
$$

The dimensions of the sample and the vertical displacement calculated using Eq. 3.3 and 3.4 are shown Table 3.7.

Table 3.7 Dimensions of specimen for FEA under bending.

\begin{tabular}{ccc}
\hline $\begin{array}{c}\text { Mandrel diameter } \\
(\mathrm{mm})\end{array}$ & $\begin{array}{c}\text { Film length } \\
(\mathrm{mm})\end{array}$ & $\begin{array}{c}\text { Bending height } \\
(\mathrm{mm})\end{array}$ \\
\hline 17.78 & 23.27 & 6.59 \\
22.6 & 29.52 & 8.36 \\
29.1 & 38.09 & 10.78 \\
\hline
\end{tabular}


Also, the same material (Table 3.6) properties were used for FEA for patterned ITO films under controlled-buckling testing. However, the values of the constraints and displacements were considered in accordance with the buckling apparatus design and dimensions. All nodes at the clamped ends were fully constrained and a vertical displacement $\left(\mathrm{u}_{\mathrm{z}}=3.5 \mathrm{~mm}\right)$ was also considered. The dimensions of the sample used in this model were $20 \mathrm{~mm}$ in length and $5 \mathrm{~mm}$ in width. In addition, in order to get a better stress distribution illustration, the sizes of the different patterns used were fifteen times that of the sizes shown in Fig. 3.1a. The error incurred, on stress values, using this size was found to be less than $1.5 \%$. This is because uniform thickness and material properties are considered when utilizing FEA.

\subsection{Life-stress model}

Furthermore, life-stress analysis was performed using Reliasoft's software (Reliasoft, Corp.). The stresses obtained from FEA and cycles to failure values calculated from mechanochemical experiments under fatigue and combined fatigue-corrosion conditions were applied to the life-stress model based on an inverse power law (IPL)-Weibull relationship to determine the characteristics life of the Weibull distribution. The IPL-Weibull relationship can be written as [99]:

$$
f(t, V)=\beta K V^{n}\left(K V^{n} t\right)^{\beta-1} e^{-\left(K V^{n} t\right)^{\beta}}
$$

where $\mathrm{V}$ represents the applied stress level and $\mathrm{t}$ is cycles to failure. $\mathrm{K}$ and $\mathrm{n}$ are model parameters to be determined, and $\beta$ represents Weibull shape parameter (slope). 


\section{CORROSION OF FLEXIBLE TRANSPARENT ELECTRODES}

\subsection{Introduction}

In this chapter the corrosion behavior of ITO film deposited at room temperature on a polymer substrate by DC magnetron sputtering is studied in different concentrations of acrylic acid in order to evaluate the stability of ITO in contact with pressure sensitive acrylic acid containing adhesives, which are employed in the fabrication of flexible optoelectronics. Electrochemical studies are useful in providing information about the processes taking place at the interface between the working electrode and the electrolyte. Hence, open-circuit potential (OCP) versus time, potentiodynamic polarization (PDP), and electrochemical impedance spectroscopy (EIS) measurements were carried out for the as-received ITO film in $0.05 \mathrm{M}$ acrylic acid in order to understand the influence of acrylic acid adhesives on the performance of ITO film. The surface morphology of ITO film after potentiodynamic polarization was characterized by scanning electron microscopy (SEM) and chemical analysis was performed by X-ray photoelectron spectroscopy (XPS). A correlation between the electrochemical behavior of the ITO film and its surface morphology is established. The parameters obtained in this chapter will be used in subsequent chapters.

\subsection{Results and discussion}

\subsubsection{In-situ electrical resistance measurements}


In-situ electrical measurements were used to assess the reliability of conductive films since electrical resistance changes during testing can be associated with surface changes due to corrosion processes [88]. Fig. 4.1 shows the normalized change in resistance. The slowest increase in resistance was exhibited in the case of $0.05 \mathrm{M}$ acrylic acid concentration and the fastest being at $0.3 \mathrm{M}$ concentration indicating corrosion of the ITO. Since corrosion activities were noted at $0.05 \mathrm{M}$, the electrochemical measurements were performed in $0.05 \mathrm{M}$ acrylic acid. The $\mathrm{pH}$ was measured to be approximately 2.8 .

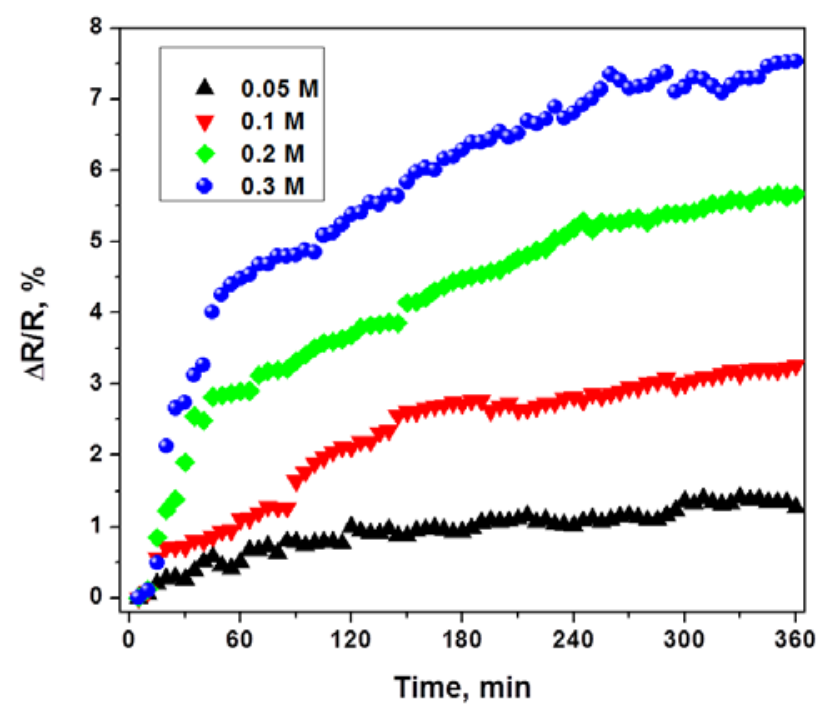

Fig. 4.1 Normalized electrical resistance vs. time for ITO film in acrylic acid of different concentrations

\subsubsection{Open-circuit potential measurement}

Fig. 4.2 shows the open-circuit potential vs. time curves for ITO film in $0.05 \mathrm{M}$ acrylic acid after different immersion times. In all cases, OCP is initially shifted to more negative 
potentials and then attain a stable potential. The steady state potentials are always more negative than the initial immersion potential $\left(E_{\mathrm{OCP}}\right.$ at $\mathrm{t}=0$ ), suggesting that before a steady state condition is achieved a surface layer has to dissolve [103]. Contamination of ITO surface layer due to air exposure has been reported by Biswas et al. [104]. They pointed out the existence of In-OH in the surface layer of ITO film and a change in $\mathrm{O}_{\text {total }} / \mathrm{In}$ ratio due to air exposure. This is also consistent with the results reported by Purvis et al. [105]. In the present study, the initial shift of OCP to negative potentials may be related to the surface layer. Hence, the steady state OCP value corresponds to free corrosion potential of bare ITO film. The steady state OCP value of ITO film is shifted to more negative values with increasing immersion time. The shift in the steady state OCP to negative values may be due to dissolution of ITO in $0.05 \mathrm{M}$ acrylic acid [106].

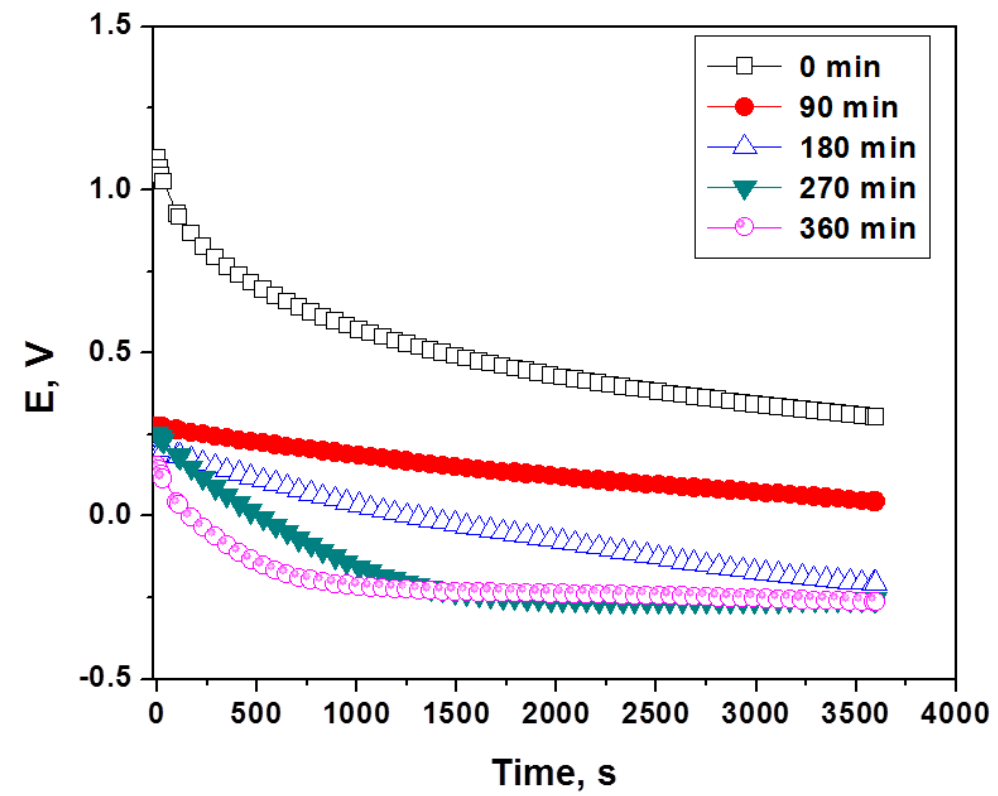

Fig. 4.2 Open-circuit potential vs. time curves for ITO film after different immersion times in $0.05 \mathrm{M}$ acrylic acid. 


\subsubsection{Potentiodynamic polarization measurement}

Typical PDP curves for ITO film measured after different immersion time in $0.05 \mathrm{M}$ acrylic acid are depicted in Fig. 4.3. The corresponding parameters determined from PDP (Tafel fit) curves are presented in Table 4.1. The corrosion potential, $\mathrm{E}_{\text {corr }}$, increases to higher values while the corrosion current density, $\mathrm{I}_{\text {corr }}$, decreases to lower values with increasing immersion time which may imply that the electro-activity of ITO film decreases with increasing immersion time. This may be due to dissociation of $\mathrm{In}^{3+}$ and $\mathrm{O}^{2-}$ which would be the first to be released from ITO [107]. The anodic and cathodic Tafel constants do not change significantly with immersion time. This observation suggests a charge transfer controlled reaction and the same mechanism is effective in both the anodic and cathodic regions [108]. In addition, the Tafel slopes obtained in the range of 56-79 mV/decade suggest one electron transfer reaction [109]. It has been reported [110] that the first ionization potential of In $(5.8 \mathrm{eV})$ is lower than that of $\mathrm{Sn}(7.3 \mathrm{eV})$ and thus indium ought to participate readily in single electron transfer processes as compared to tin. Therefore, the dissolution of ITO involves reduction of $\mathrm{In}_{2} \mathrm{O}_{3}$ into metallic indium. Furthermore, the cathodic polarization curves (Fig. 4.3) are assumed to represent the cathodic decomposition of ITO through a reduction reaction [111]. When the ITO electrode is cathodically polarized in acrylic acid, $\mathrm{In}_{2} \mathrm{O}_{3}$ may be reduced to metallic indium [107]. The anodic polarization curves are assumed to represent the oxygen evolution reaction through an oxidation reaction. Produced oxygen ions are removed from ITO resulting in the formation of metallic indium [112]. 


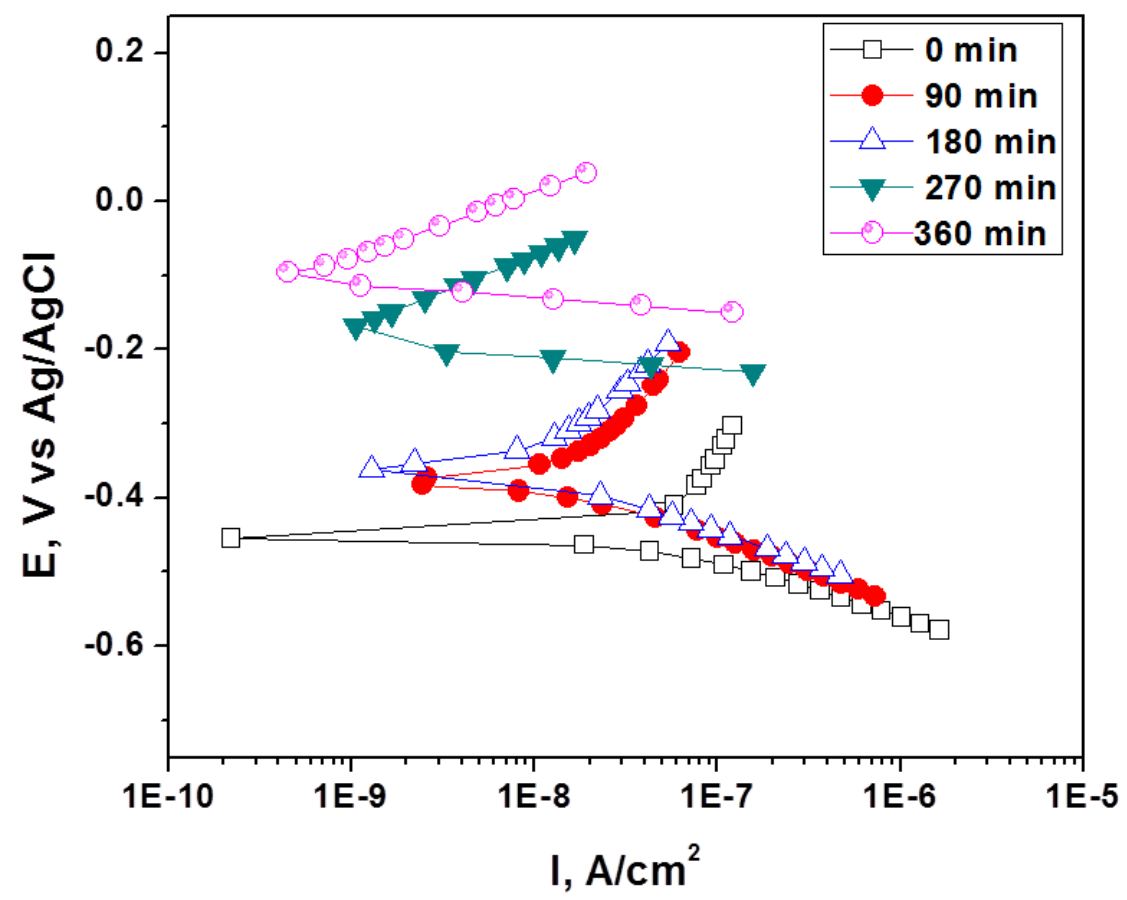

Fig. 4.3 Potentiodynamic polarization (Tafel fit) curves for ITO film after different immersion times in $0.05 \mathrm{M}$ acrylic acid.

Table 4.1 Potentiodynamic polarization parameters for ITO film after different immersion times in $0.05 \mathrm{M}$ acrylic acid

\begin{tabular}{ccccc}
\hline $\begin{array}{c}\text { Immersion } \\
\text { time }(\mathrm{min})\end{array}$ & $\begin{array}{c}\mathrm{E}_{\text {corr }}(\mathrm{mV}) \\
\text { vs Ag/AgCl }\end{array}$ & $\begin{array}{c}\mathrm{I}_{\text {corr } \times 10^{-3}} \\
\left(\mu \mathrm{A} / \mathrm{cm}^{2}\right)\end{array}$ & $\mathrm{B}_{\mathrm{a}}(\mathrm{mV})$ & $\mathrm{B}_{\mathrm{c}}(\mathrm{mV})$ \\
\hline \hline 0 & -454.91 & 64.639 & 79.37 & 73.716 \\
90 & -378.15 & 18.446 & 62.848 & 60.648 \\
180 & -359.31 & 11.635 & 60.958 & 59.03 \\
270 & -190.33 & 0.63665 & 58.99 & 57.838 \\
360 & -105.02 & 0.49348 & 56.3 & 48.163 \\
\hline
\end{tabular}




\subsubsection{Scanning electron microscopy}

A typical SEM image of amorphous ITO (as received) is shown in Fig. 4.4a. Figs. 4.4b-f show the SEM images of ITO and morphological changes of the ITO surface after potentiodynamic polarization measurements for different immersion times in $0.05 \mathrm{M}$ acrylic acid. The SEM study confirms the extent of corrosion of ITO film in $0.05 \mathrm{M}$ acrylic acid and it is in agreement with the potentiodynamic polarization measurements which suggest the continuous dissolution of ITO. As dissolution of ITO increases the film becomes more porous. 


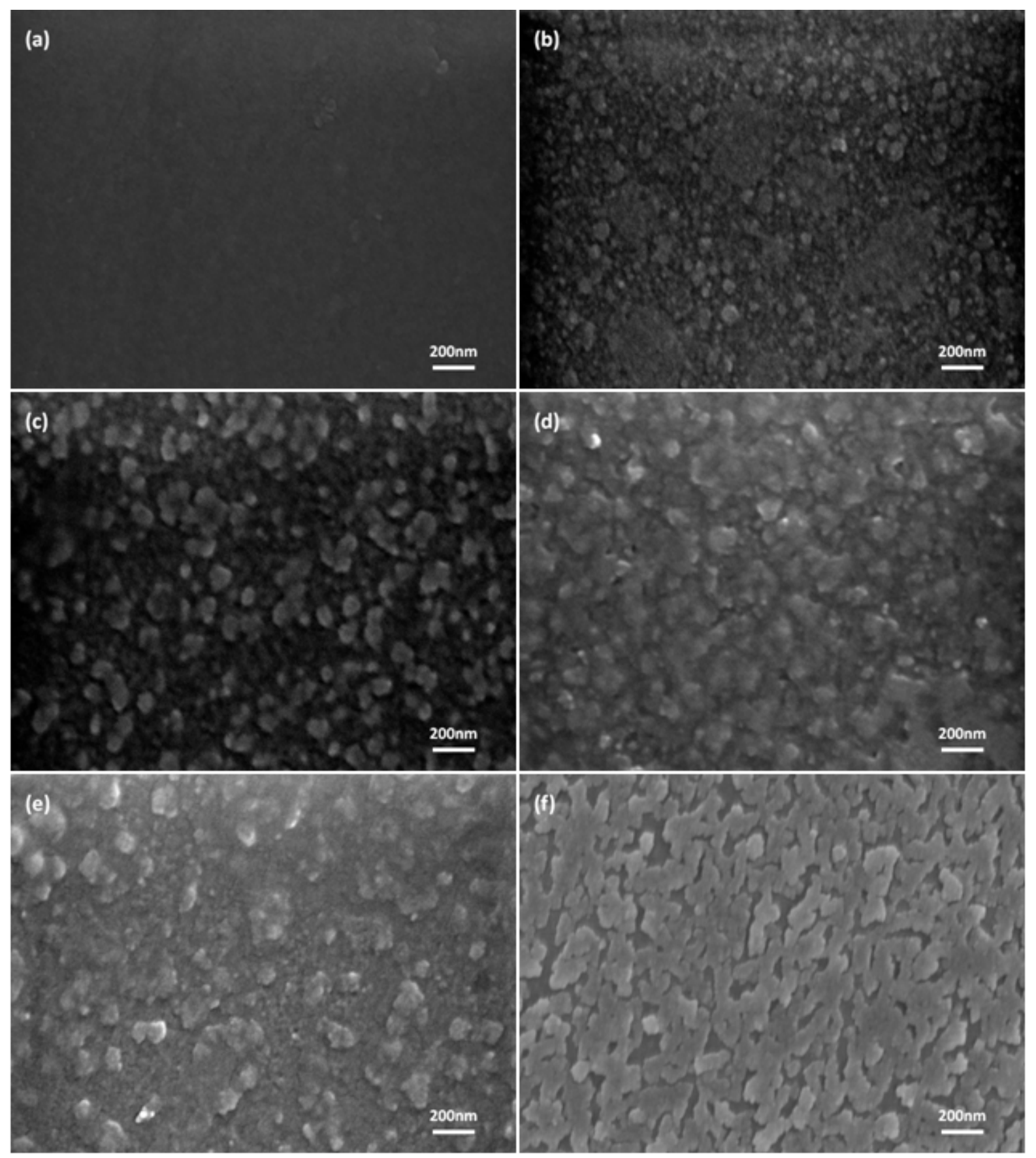

Fig. 4.4 SEM image of ITO film for (a) as received ITO, (b) 0 min, (c) 90 min, (d) 180 min, (e) $270 \mathrm{~min}$, and (f) $360 \mathrm{~min}$ of immersion in $0.05 \mathrm{M}$ acrylic acid and after electrochemical measurements. 


\subsubsection{X-ray photoelectron spectroscopy}

To understand the corrosion phenomena, the surface of ITO film was characterized using XPS. XPS spectra of In 3d levels from the as received and corroded ITO films are shown in Fig. 4.5. The asymmetry of these peaks indicates that multiple species exist. The In $3 \mathrm{~d}$ region was deconvoluted to obtain two peaks which represent the contributions from $\operatorname{In}^{0}$ and $\operatorname{In}^{3+}$ states. These peaks were assigned to $443.8 \mathrm{eV}$ and $444.9 \mathrm{eV}$, respectively, with $\mathrm{FWHM} \leq 1.5 \mathrm{eV}[113$, 114]. The relative concentrations of the indium species are presented in Table 4.2. These results show that in the first 90 minutes of immersion time, nearly half of the initial amount of $\operatorname{In}^{0}$ is removed when compared with the as received ITO film. This may be due to dissolution of metallic indium after longer immersion time in acrylic acid which is suggested by the polarization analysis. The slight increase of $\operatorname{In}^{0}$ for 360 minutes of immersion may be attributed to metallic indium from the newly exposed surfaces that are visible in the SEM micrograph.

Table 4.2 Relative concentrations of indium species for ITO film after different immersion times in $0.05 \mathrm{M}$ acrylic acid and electrochemical measurements.

\begin{tabular}{cccc}
\hline $\begin{array}{c}\text { Immersion time } \\
(\mathrm{min})\end{array}$ & $\% \mathrm{In}^{0}$ & $\% \mathrm{In}^{3+}$ & $\% \mathrm{In}^{3+} / \mathrm{In}^{0}$ \\
\hline \hline $\begin{array}{c}\text { As received (No } \\
\text { EIS) }\end{array}$ & 45.5 & 54.5 & 1.2 \\
0 & 41.6 & 58.5 & 1.4 \\
90 & 26.3 & 73.7 & 2.8 \\
180 & 23.7 & 76.3 & 3.2 \\
270 & 14.4 & 85.7 & 6 \\
360 & 24.4 & 75.6 & 3.1 \\
\hline
\end{tabular}



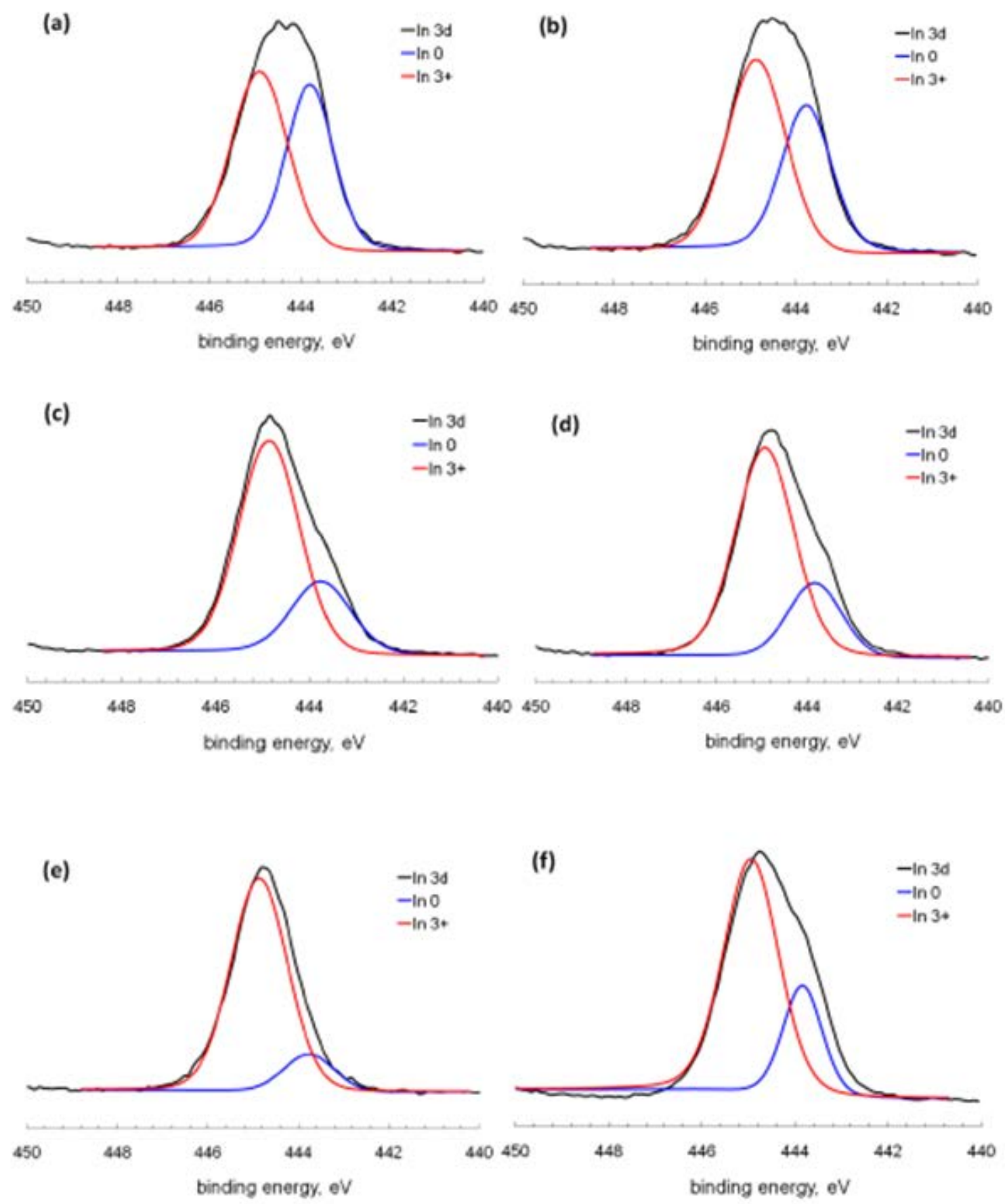

Fig. 4.5 XPS spectrum of ITO film for (a) as received ITO, (b) 0 min, (c) $90 \mathrm{~min}$, (d) $180 \mathrm{~min}$, (e) $270 \mathrm{~min}$, and (f) $360 \mathrm{~min}$ of immersion in $0.05 \mathrm{M}$ acrylic acid and after electrochemical measurements. 


\subsubsection{Electrochemical impedance spectroscopy measurement}

The experimental data were fitted to an equivalent circuit (Fig. 4.6) consisting of resistances (R), capacitances (C) and/or constant phase elements (CPE). In this circuit, $R_{s}$ is the solution resistance, estimated by fitting the impedance data, approximately $100 \Omega . \mathrm{C}_{\mathrm{sc}}$ and $\mathrm{R}_{\mathrm{sc}}$ are space charge layer capacitance and space charge layer resistance, $\mathrm{C}_{\mathrm{dl}}$ and $\mathrm{R}_{\mathrm{ct}}$ are the double layer capacitance and charge transfer resistance, respectively. The equivalent circuit (Fig. 4.6) which simulates the ITO film/electrolyte interface is proposed by Serantoni et al. [115] and Lin et al. [116]. $\mathrm{C}_{\mathrm{sc}}$ represents a pseudo-capacitor due to charge accumulated in the depletion/accumulation layer that leads to band bending inside the semiconductor and $\mathrm{R}_{\mathrm{sc}}$ is the resistance due to the movement of electrons inside the space charge layer and depends on the potential drop inside the semiconductor and the flowing current [115]. $R_{c t}$ is considered due to the resistance to the charge transfer between the semiconductor and the solution and $\mathrm{C}_{\mathrm{dl}}$ is attributed to the charge layer on the surface of the semiconductor and the counter-ion's layer on the solution side accumulated to neutralize the first layer due to the electrolyte being used [115]. A constant phase element (CPE) is used, instead of a capacitor, to take into account any nonhomogeneity of the double layer. The impedance of this element is expressed as follows [117]:

$$
\mathrm{Z}_{\mathrm{CPE}}=1 / \mathrm{C}(\mathrm{i} \omega)^{\mathrm{n}}
$$

where, $n$ is a measure of capacitive behavior of the film. When $n=1$, the CPE resembles to a perfect capacitor and the Bode phase angle for this pure capacitive behavior is $-90^{\circ}$. When $\mathrm{n}=0$, the CPE resembles to a perfect resistor and the Bode phase angle is $0^{\circ}[118,119]$. 


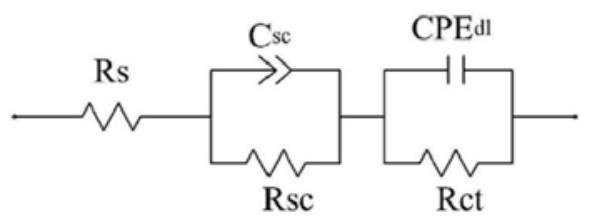

Fig. 4.6 The equivalent circuit used to fit the impedance data for ITO film after different immersion times in $0.05 \mathrm{M}$ acrylic acid.

Figs. 4.7-4.9 show the Nyquist and Bode plot for the ITO film after different immersion times in $0.05 \mathrm{M}$ acrylic acid, respectively. The Nyquist plot (Fig. 4.7) shows two time constants. The time constant observed at low frequency region is attributed to the charge transfer reaction taking place at the ITO film/electrolyte interface. The time constant resolved at high frequency region (Fig. 4.7 inset) is attributed to the surface charge layer, which is representative of the semiconductor ITO film properties.

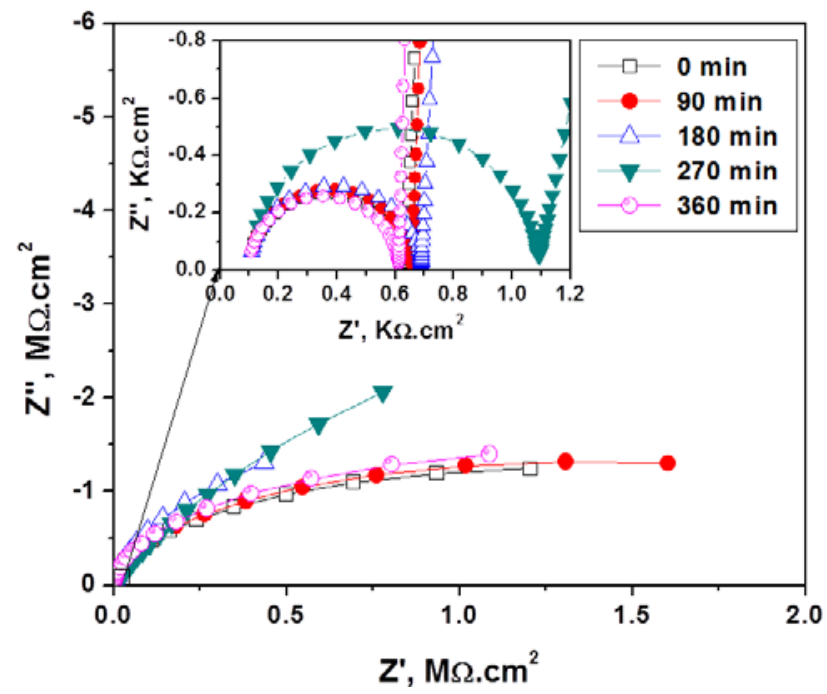

Fig. 4.7 Nyquist plot for ITO film after different immersion times in 0.05 M acrylic acid. 
Table 4.3 shows the fitting parameters for the elements in the equivalent circuit (Fig. 4.6) obtained by applying the Non-linear Least Square technique on the EIS data. The charge transfer resistance $R_{c t}$, is an important corrosion parameter and is always inversely proportional to the corrosion current density and thus the corrosion rate [120]. $R_{c t}$ increases with increasing immersion time. The increase in the charge transfer resistance indicates that the redox reaction at the interface is hindered with immersion time. This observation suggests that the dynamic processes taking place at the ITO surface may be slowed down due to the loss of charge carrier at ITO film, i.e. the dissolution of metallic indium through the reduction of $\operatorname{In}_{2} \mathrm{O}_{3}$. The slight decrease in $\mathrm{R}_{\mathrm{ct}}$ observed for $360 \mathrm{~min}$ is consistent with the XPS results. The space charge layer resistance, $\mathrm{R}_{\mathrm{sc}}$, which is related to the stability of ITO film, increases with increasing immersion time. This can be related to the decrease in the electro-activity of ITO film by the expulsion of oxygen counter ions.

Table 4.3 The parameters of equivalent circuit obtained by fitting the experimental data of EIS for ITO film after different immersion times in $0.05 \mathrm{M}$ acrylic acid $\left(\mathrm{R}_{\mathrm{s}}=100 \Omega \mathrm{cm}^{2}\right)$.

\begin{tabular}{cccccc}
\hline $\begin{array}{c}\text { Immersion time } \\
(\mathrm{min})\end{array}$ & $\begin{array}{c}\mathrm{C}_{\mathrm{sc}} \\
\left(\mathrm{F} \mathrm{cm}^{-2}\right)\end{array}$ & $\begin{array}{c}\mathrm{R}_{\mathrm{sc}} \\
\left(\Omega \mathrm{cm}^{2}\right)\end{array}$ & $\begin{array}{c}\mathrm{CPE}_{\mathrm{dl}} \\
\left(\Omega^{-1} \mathrm{~S}^{\mathrm{n}} \mathrm{cm}^{-2}\right)\end{array}$ & $\mathrm{n}_{\mathrm{dl}}$ & $\begin{array}{c}\mathrm{R}_{\mathrm{ct}} \\
\left(\Omega \mathrm{cm}^{2}\right)\end{array}$ \\
\hline \hline 0 & $1.670 \times 10^{-9}$ & 537.9 & $6.185 \times 10^{-6}$ & 0.98 & $2.595 \times 10^{6}$ \\
90 & $1.353 \times 10^{-9}$ & 557.2 & $4.569 \times 10^{-6}$ & 0.98 & $2.736 \times 10^{6}$ \\
180 & $2.456 \times 10^{-9}$ & 588 & $10.033 \times 10^{-6}$ & 0.97 & $5.158 \times 10^{6}$ \\
270 & $0.987 \times 10^{-9}$ & 986.4 & $4.891 \times 10^{-6}$ & 0.88 & $5.056 \times 10^{6}$ \\
360 & $2.491 \times 10^{-9}$ & 512.4 & $6.792 \times 10^{-6}$ & 0.98 & $2.986 \times 10^{6}$ \\
\hline
\end{tabular}


The double layer capacitance $\mathrm{C}_{\mathrm{dl}}$, is in the range of $\mathrm{mF}$ as ITO film employed in the present study is amorphous in nature. It has been reported that the conductivity of amorphous phase of ITO is comparatively lower than its crystalline phase [121].

The transition from conductive state to resistive state with increasing immersion time can be observed on the Bode frequency plot (Fig. 4.8) at the low frequency region. The increase in the resistance with immersion time at the low frequency region in the Bode frequency plot, which is representative of the ITO film properties, confirms the degradation of ITO film conductivity with immersion time in acrylic acid.

Fig. 4.9 shows the Bode phase angle plot of ITO film after different immersion time in $0.05 \mathrm{M}$ acrylic acid. Double peaks in the phase-angle curve suggest that two time constants existed for the electrochemical process at the electrolyte/film interface [122].

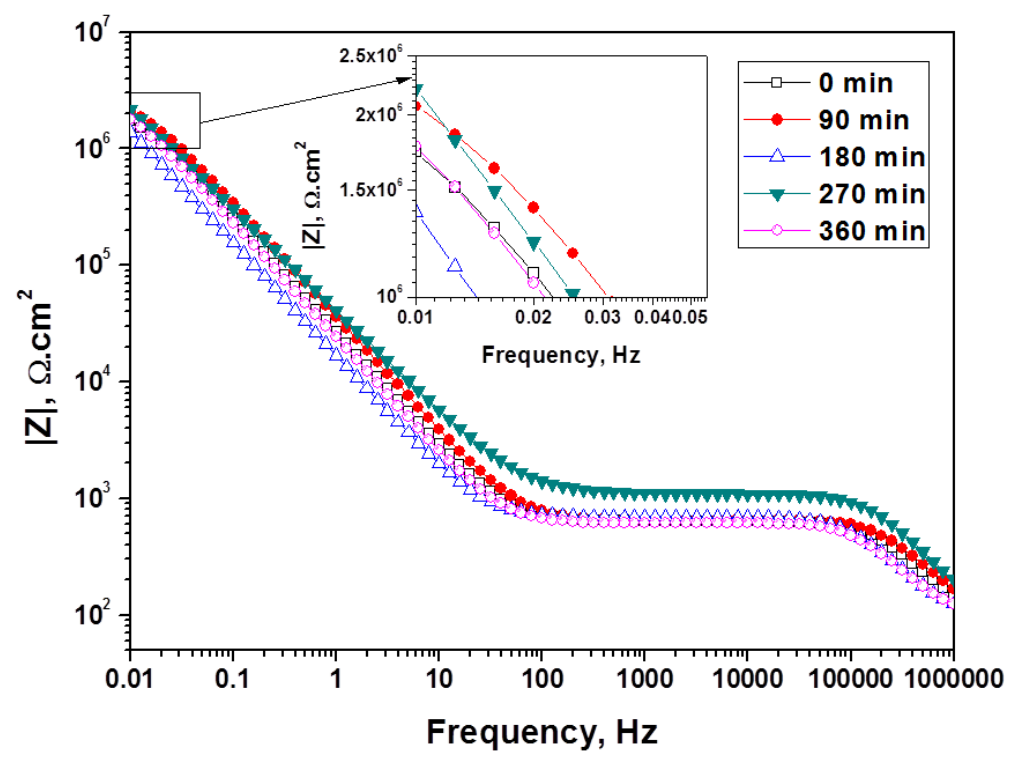

Fig. 4.8 Bode frequency plot for ITO film after different immersion times in 0.05 M acrylic acid. 


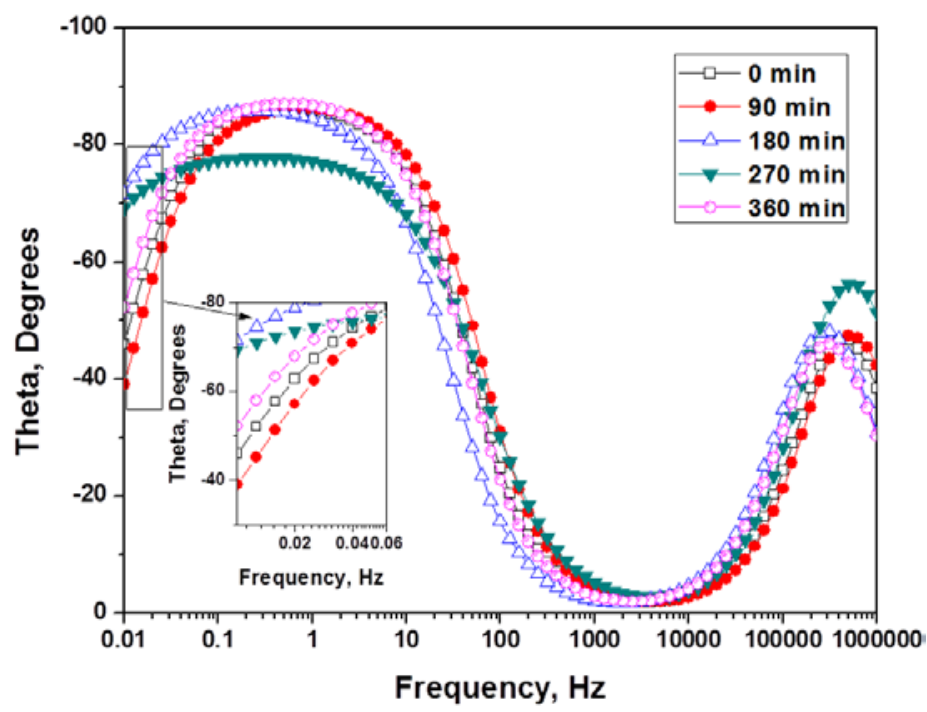

Fig. 4.9 Bode phase angle plot for ITO film after different immersion times in 0.05 M acrylic acid. 


\section{MECHANO-CHEMICAL DEGRADATION OF FLEXIBLE TRANSPARENT ELECTRODES}

\subsection{Introduction}

In this chapter the effects of corrosion, applied mechanical strain, film thickness, and number of bending cycles on the electrical and structural integrity of indium tin oxide (ITO) and carbon nanotube (CNT) films, both coated on polyethylene terephthalate (PET) substrates, are investigated using design of experiment methods.

In-situ electrical resistance monitoring was conducted during corrosion, bending, fatigue, bending-corrosion, and fatigue-corrosion experiments in order to study the combined action and further accumulation of both repeated mechanical loading and corrosion which can aggravate the loss of functionality of the electrodes. Crack density calculation was performed on images acquired using optical microscopy. In addition, scanning electron microscopy was conducted in order to determine failure mechanisms.

\subsection{Results and discussion}

\subsubsection{In-situ electrical resistance measurements}

In order to investigate the effect of acid concentration, applied strain, film thickness, and number of cycles on the mechano-chemical degradation of the transparent electrodes under static and dynamic loading, in-situ electrical measurements were conducted. Electrical resistance 
changes of the transparent conducting layer, during testing, can be associated with loss of coating functionality [88]. The normalized electrical resistance versus number of cycles and/or time plots under corrosion, bending, bending-corrosion, fatigue, and fatigue-corrosion is shown in Fig. 5.1.

During the initial stages of fatigue-only case, two regimes of electrical resistance increase are observed. At first an increase of electrical resistance due to changes in dimensions of the compliant substrate, until an equilibrium size is attained, and then a gradual linear increase that may be due to cracking of ITO. Gorkhali et al. [80] reported that the initial increase of electrical resistance for an applied strain of $1.5 \%$ is $50-100$ cycles. In this study the initial increase, for 0.5\% lower applied strain, is observed to lie around 800-1000 cycles (Fig. 5.1f). Also, in all cases, the change in electrical resistance for specimens subjected to fatigue-corrosion is observed to be the highest. On the other hand the lowest change is observed in the case of corrosion-only experimental conditions. Furthermore, the critical number of cycles (Fig. 5.2) for initial loss of functionality, under fatigue and combined fatigue-corrosion of ITO films, is determined from an increase of $10 \%$ in electrical resistance (Fig. 5.1). 
(a)

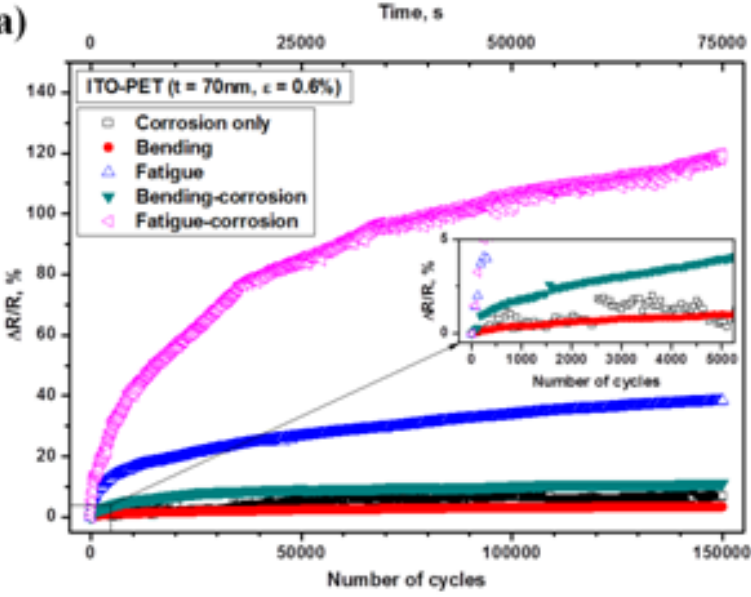

(c)

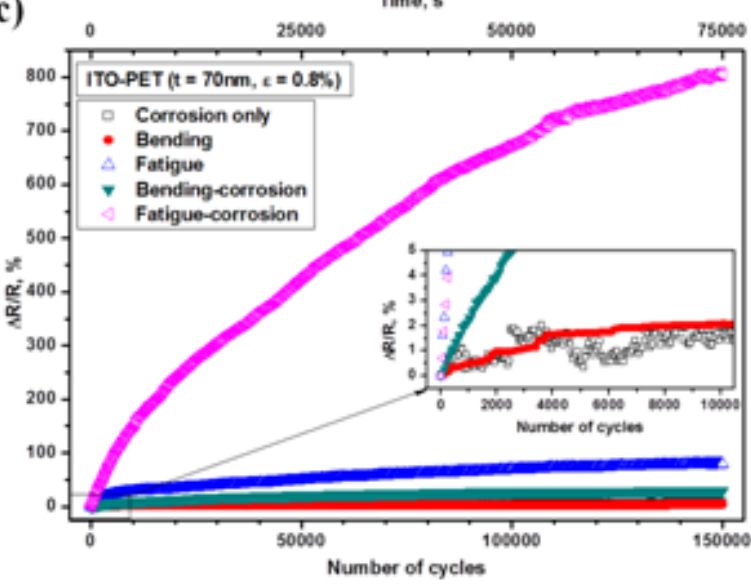

(e)

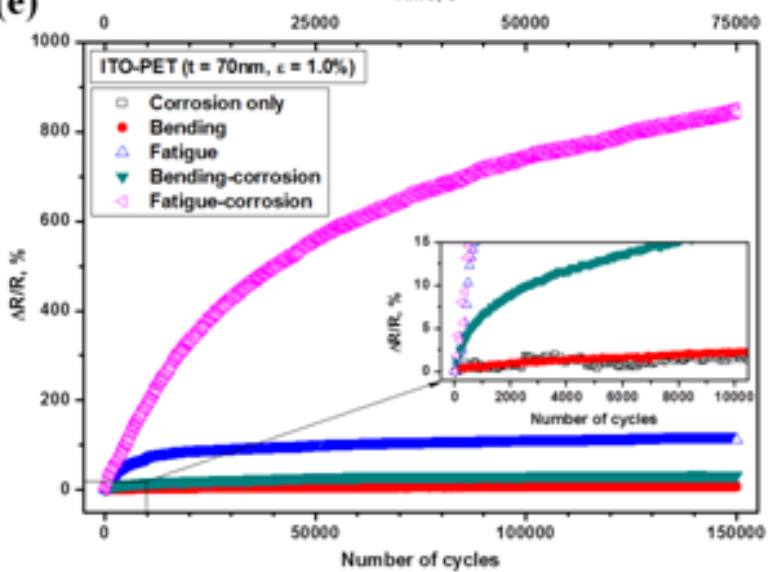

(b)

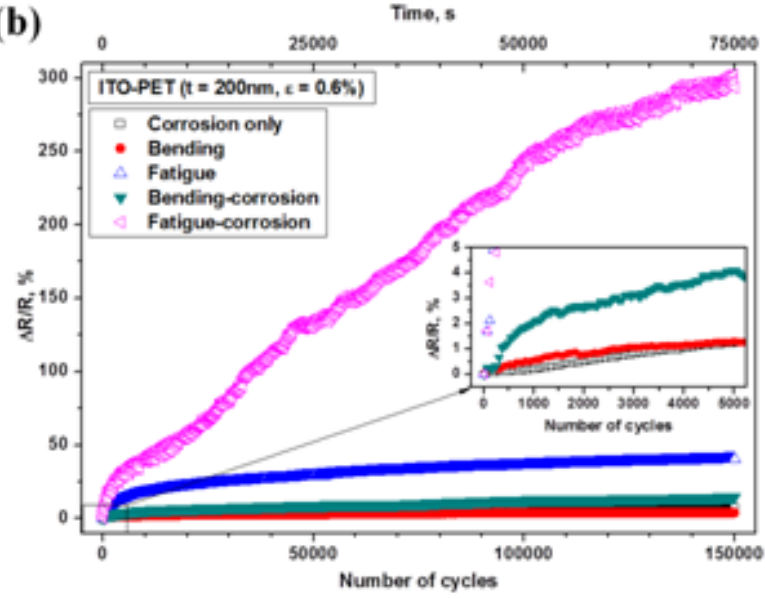

(d)

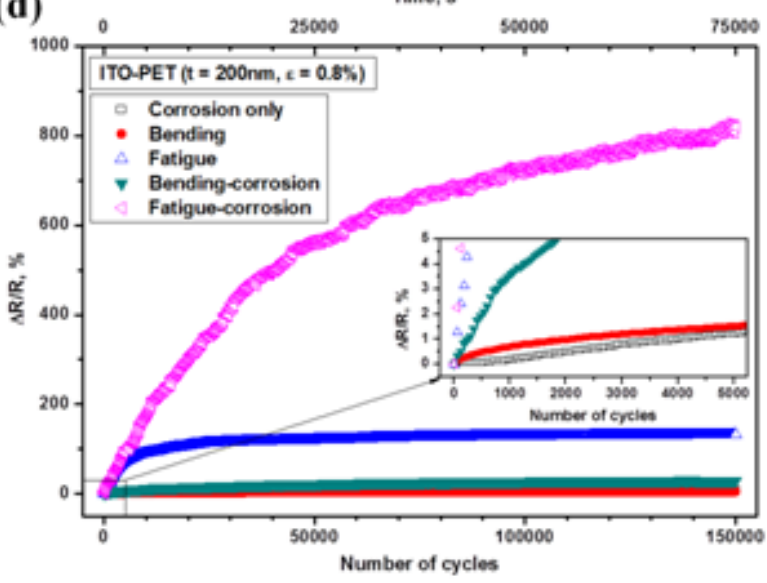

(f)

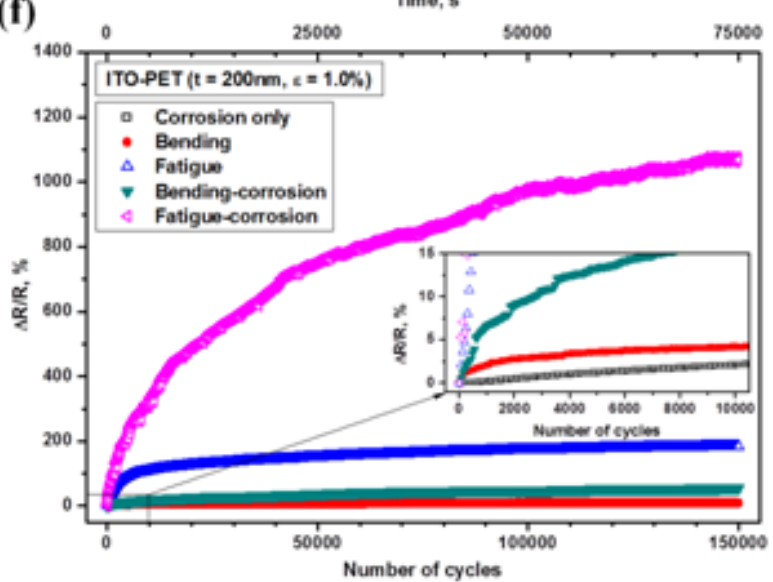

Fig. 5.1 Normalized electrical resistance versus experimental number of cycles and/or time for ITO-coated PET at applied strain and film thickness of: (a) $70 \mathrm{~nm}$ and $0.6 \%$, (b) $200 \mathrm{~nm}$ and 0.6\%, (c) $70 \mathrm{~nm}$ and $0.8 \%$, (d) $200 \mathrm{~nm}$ and $0.8 \%$, (e) $70 \mathrm{~nm}$ and 1.0\%, (f) $200 \mathrm{~nm}$ and 1.0\%, respectively. 
(a)

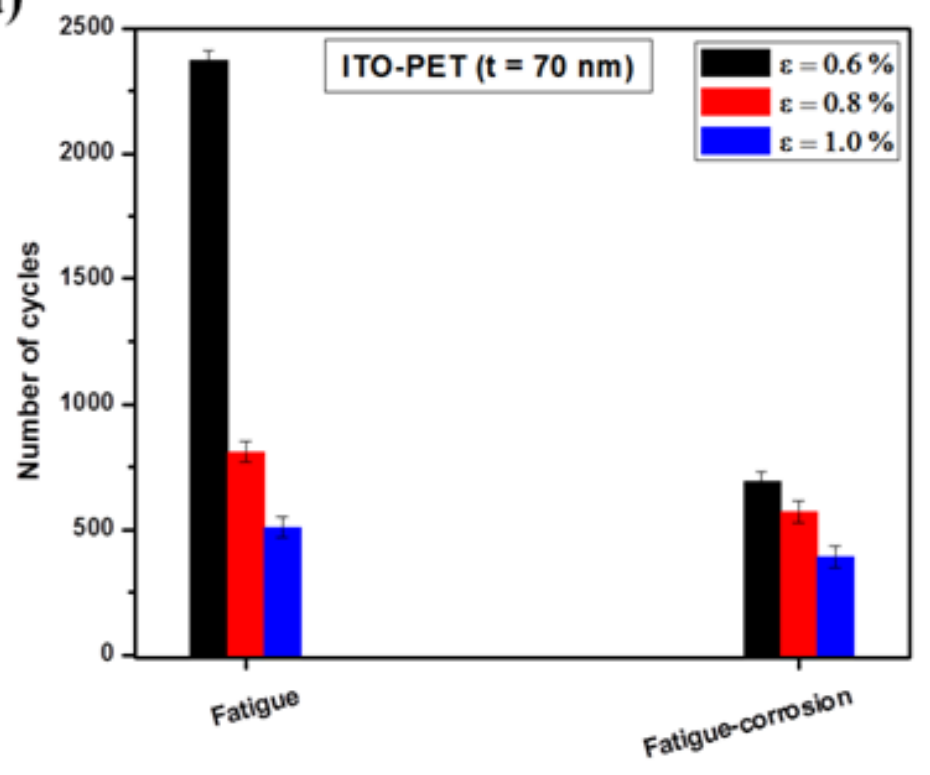

(b)

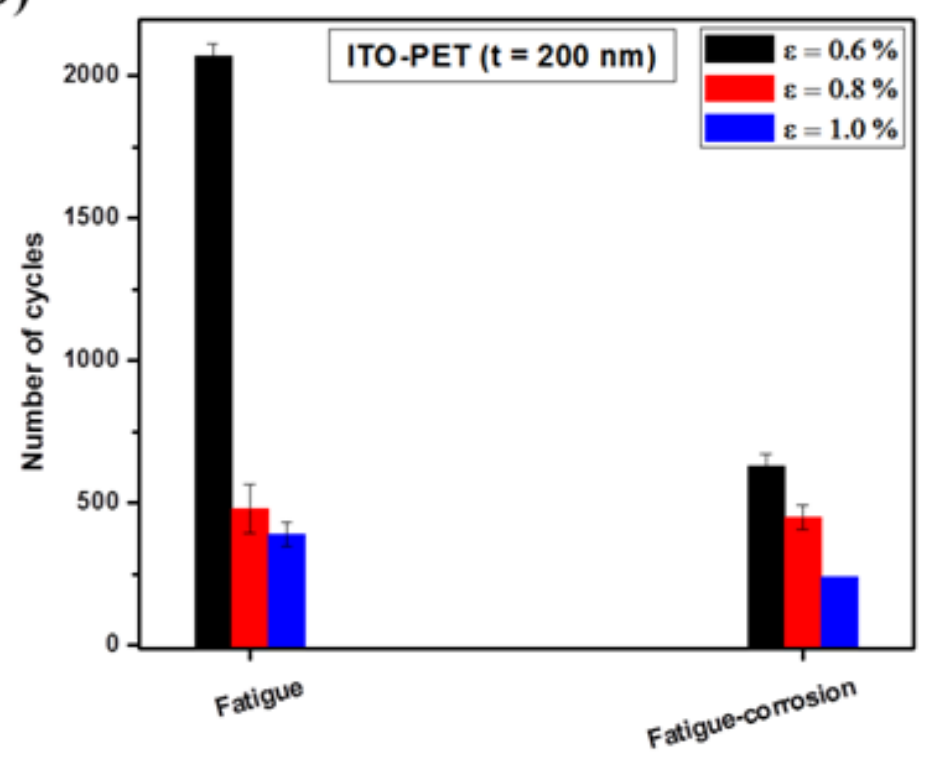

Fig. 5.2 Critical number of cycles for fatigue and fatigue-corrosion of ITO-coated PET for film thickness of (a) $70 \mathrm{~nm}$ and (b) $200 \mathrm{~nm}$. 
In general, specimens under fatigue-corrosion degradation are observed to exhibit a lower critical number of cycles for both 70 and $200 \mathrm{~nm}$ thick ITO films. This denotes the significance of the combined effect of fatigue and corrosion, which can be used as an accelerated degradation experimental protocol that can aid towards identifying the interrelation of the critical factors that influence the long-term reliability of the device component.

Fig. 5.3 shows the effect of applied strain on the electrical degradation of ITO films at different degradation conditions. A common observation is that, for each condition and film thickness, the higher the applied strain the larger the positive change in electrical resistance. For example, we observe that at 150,000 cycles the change in ITO (200 nm) electrical resistance during fatigue-corrosion is $300 \%$ for $0.6 \%$ strain and $1107 \%$ for $1.0 \%$ applied strain. This is denoting a more than a threefold increase. Such phenomenon can be related to the increased film crack formation as the applied strain increases. 
(a)

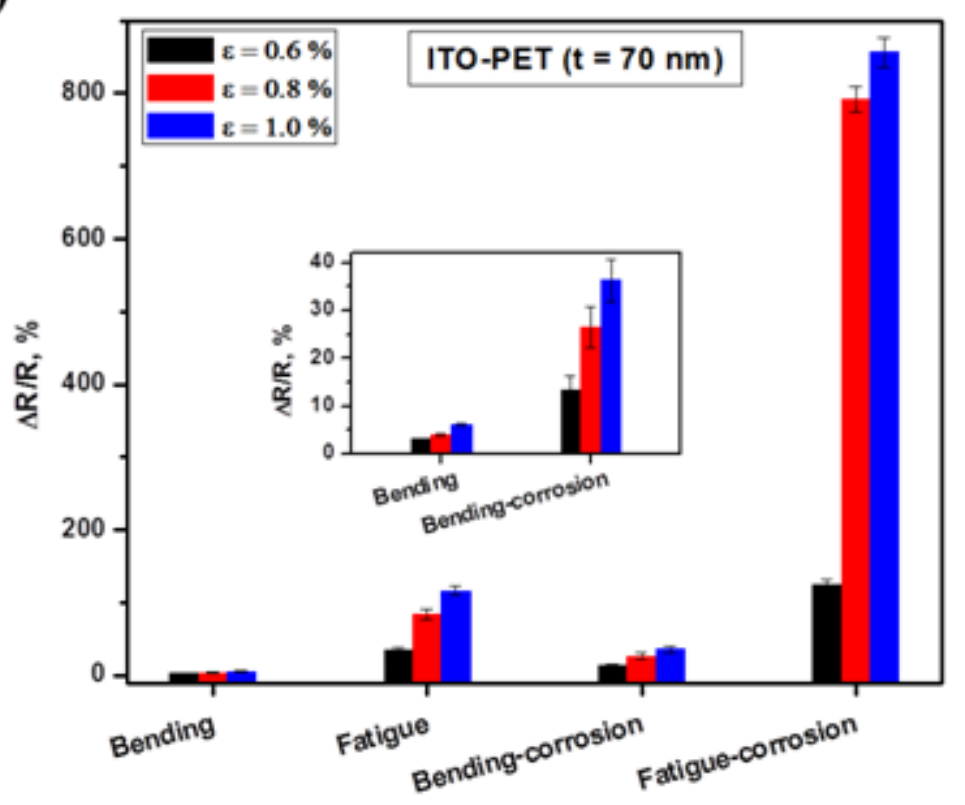

(b)

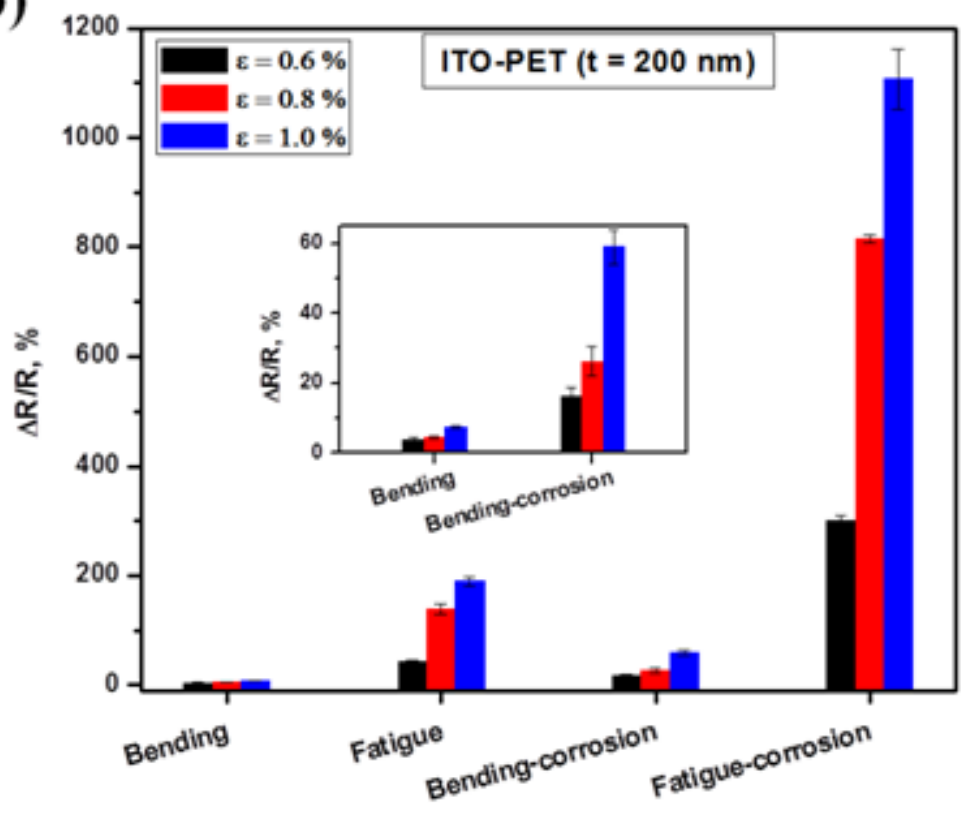

Fig. 5.3 Effect of strain on normalized electrical resistance for different testing conditions at 150,000 number of cycles for film thickness of (a) $70 \mathrm{~nm}$ and (b) $200 \mathrm{~nm}$. 
Also, the effect of film thickness on the electrical degradation of ITO films was investigated at different conditions, as shown in Fig. 5.4. Higher change in electrical resistance values are observed for the thicker films, suggesting that they are influenced, more than their thinner counterparts, by the pure mechanical or the mechano-chemical imposed degradation. It is believed that that the higher concentration of pre-existing flaws and defects in the thicker layer may play a role for such a response. On the other hand, it has been reported in the past that thinner layers of ITO can better withstand applied strain than thicker layers [71]. However, the increase in resistance is not observed to be as large as the one observed during the application of different strains. This indicates the higher significance of applied strain as a degradation factor. 
(a)

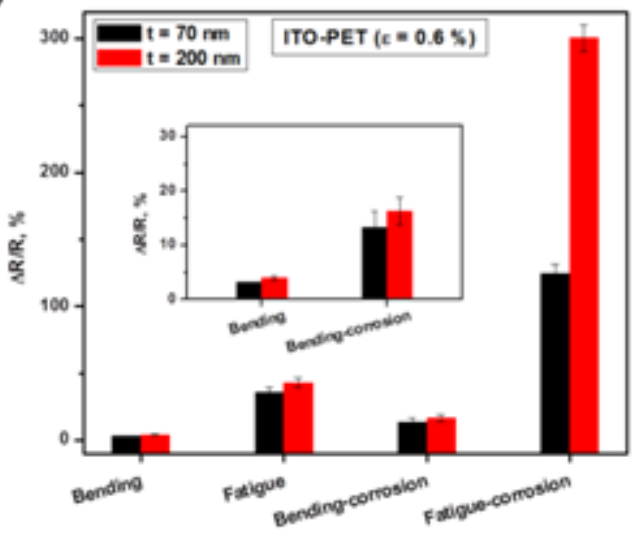

(b)

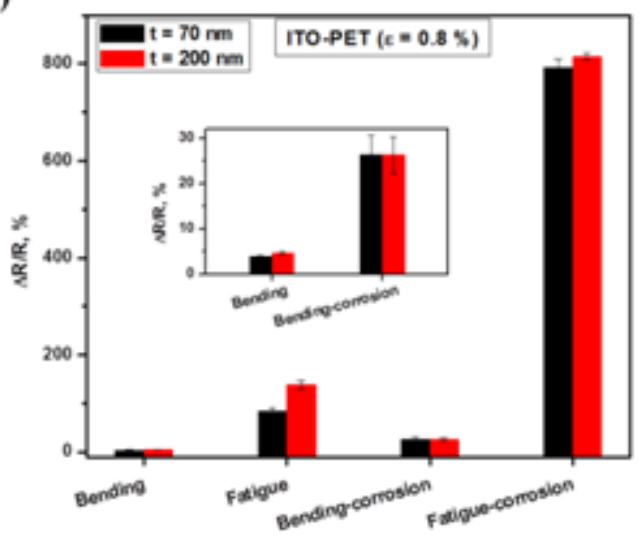

(c)

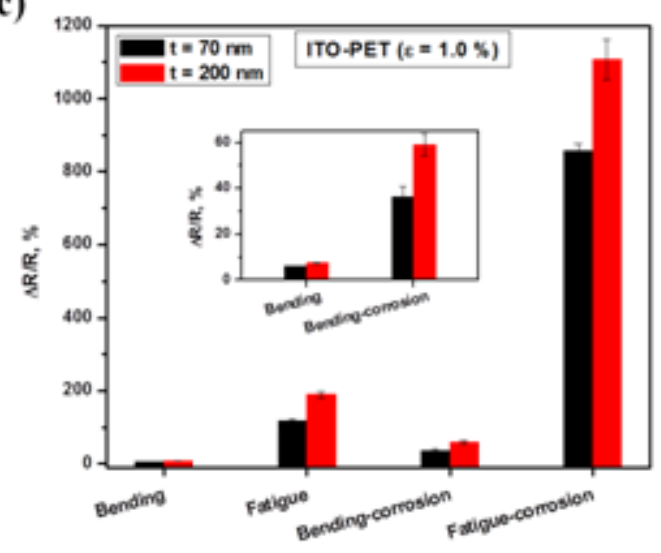

Fig. 5.4 Effect of film thickness on normalized electrical resistance for different testing conditions at 150,000 number of cycles at applied strains (a) $0.6 \%$, (b) $0.8 \%$, and (c) $1.0 \%$. 
Furthermore, CNT films are tested under the same conditions. In contrast to the ITO film behavior, CNT-based electrodes exhibit a different trend as shown in Fig. 5.5.
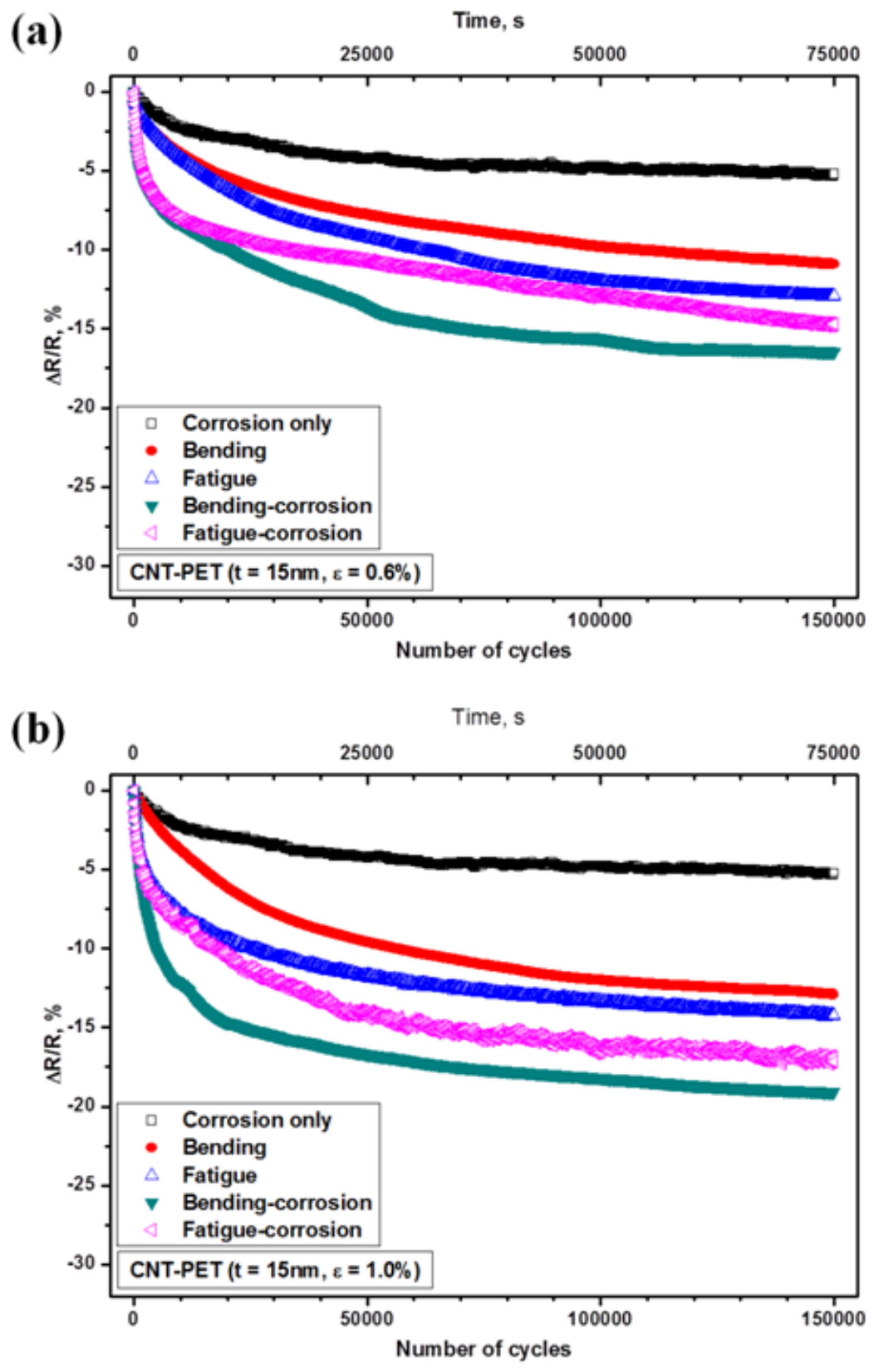

Fig. 5.5 Normalized electrical resistance versus experimental time/number of cycles for CNTdeposited PET at applied strain of: (a) $0.6 \%$ and (b) $1.0 \%$. 
The change in electrical resistance is observed to be negative, indicating enhanced electrical conductivity of the CNT films. In the presence of a combined mechano-corrosive environment this negative change becomes more pronounced. It is believed that the observed behavior is due to the effect of the acrylic acid solution. The role of acid treatment on CNT networks has been reported in the past. The acid further treats and enhances the metallicity of the CNTs as suggested by both Geng et al. and Nirmalraj et al. [123, 124]. In addition, Hecht et al. [125] reported that immersion of CNT films on polycarbonate substrates in $0.1 \mathrm{M}$ acrylic acid solution leads to a negative change in electrical resistance (-2.5\%). In this study, the combined effect of fatigue and acidic corrosion leads to increased binder cracking and, therefore, to a larger CNT area exposure. This in turn leads to an increased number of acid-treated CNT bundles and, therefore, higher film conductivity. However, the change in electrical resistance value is not significant as compared to the ITO film case. It is therefore suggested that CNT films can better withstand externally applied strains than their ITO counterparts under the combined actions of fatigue and corrosion.

\subsubsection{Design of experiments}

A further investigation of the effects of corrosion, applied strain, film thickness, and number of cycles (NOC) on the electrical degradation of ITO films was conducted using DOE methods. Table 5.1 shows the analysis of variance (ANOVA) for the main effects and their twoway and three-way interactions under fatigue and fatigue-corrosion. 
Table 5.1 Analysis of variance (ANOVA) for fatigue and fatigue-corrosion

\begin{tabular}{|lrrrrrr|}
\hline Source & DF & Seq SS & Adj SS & Adj MS & F & P \\
\hline Conc. & 1 & 1470543 & 1470543 & 1470543 & 1548.74 & 0.0000 \\
Strain & 2 & 730137 & 730137 & 365068 & 384.48 & 0.0000 \\
Thickness & 1 & 58334 & 58334 & 58334 & 61.44 & 0.0000 \\
NOC & 3 & 1056107 & 1056107 & 352036 & 370.76 & 0.0000 \\
Conc.*Strain & 2 & 396287 & 396287 & 198144 & 208.68 & 0.0000 \\
Conc.*Thickness & 1 & 16322 & 16322 & 16322 & 17.19 & 0.0060 \\
Conc.*NOC & 3 & 565061 & 565061 & 188354 & 198.37 & 0.0000 \\
Strain*Thickness & 2 & 9949 & 9949 & 4975 & 5.24 & 0.0480 \\
Strain*NOC & 6 & 261251 & 261251 & 43542 & 45.86 & 0.0000 \\
Thickness*NOC & 3 & 19637 & 19637 & 6546 & 6.89 & 0.0230 \\
Conc.*Strain*Thickness & 2 & 6410 & 6410 & 3205 & 3.38 & 0.1040 \\
Conc.*Strain*NOC & 6 & 145552 & 145552 & 24259 & 25.55 & 0.0010 \\
Conc.*Thickness*NOC & 3 & 5585 & 5585 & 1862 & 1.96 & 0.2210 \\
Strain*Thickness*NOC & 6 & 8380 & 8380 & 1397 & 1.47 & 0.3260 \\
Error & 6 & 5697 & 5697 & 950 & & \\
Total & 47 & 4755252 & & & & \\
S= 30.8141 $\quad$ R-sq=99.88\% & R-sq(adj)=99.06\% & & & \\
\hline
\end{tabular}

All design factors and their two-way interactions are found to be significant since their Pvalues are less than the confidence level $(\alpha=0.05)$. However, three-way interactions involving thickness are observed to be insignificant. From the main effects plots (Fig. 5.6), the most significant effects are acid concentration, strain, and NOC. High initial rate of change of ITO electrical resistance is observed for both strain and NOC. The significant contribution of corrosion on the change in electrical resistance increase is shown by the two-way interaction plots (Fig. 5.7). 


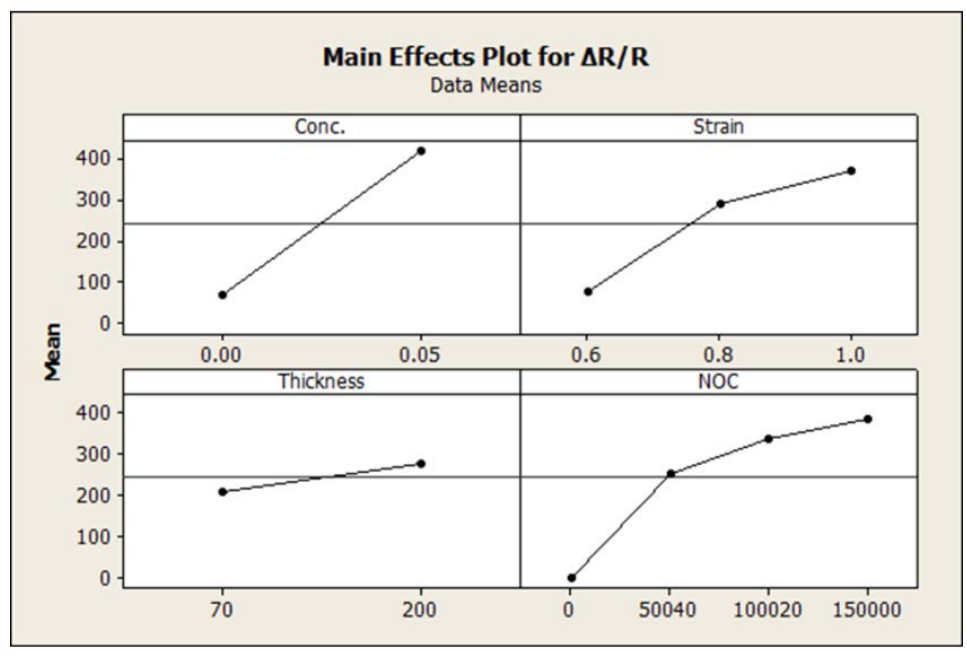

Fig. 5.6 Main effects plot for change in electrical resistance for ITO films under fatigue and fatigue-corrosion.

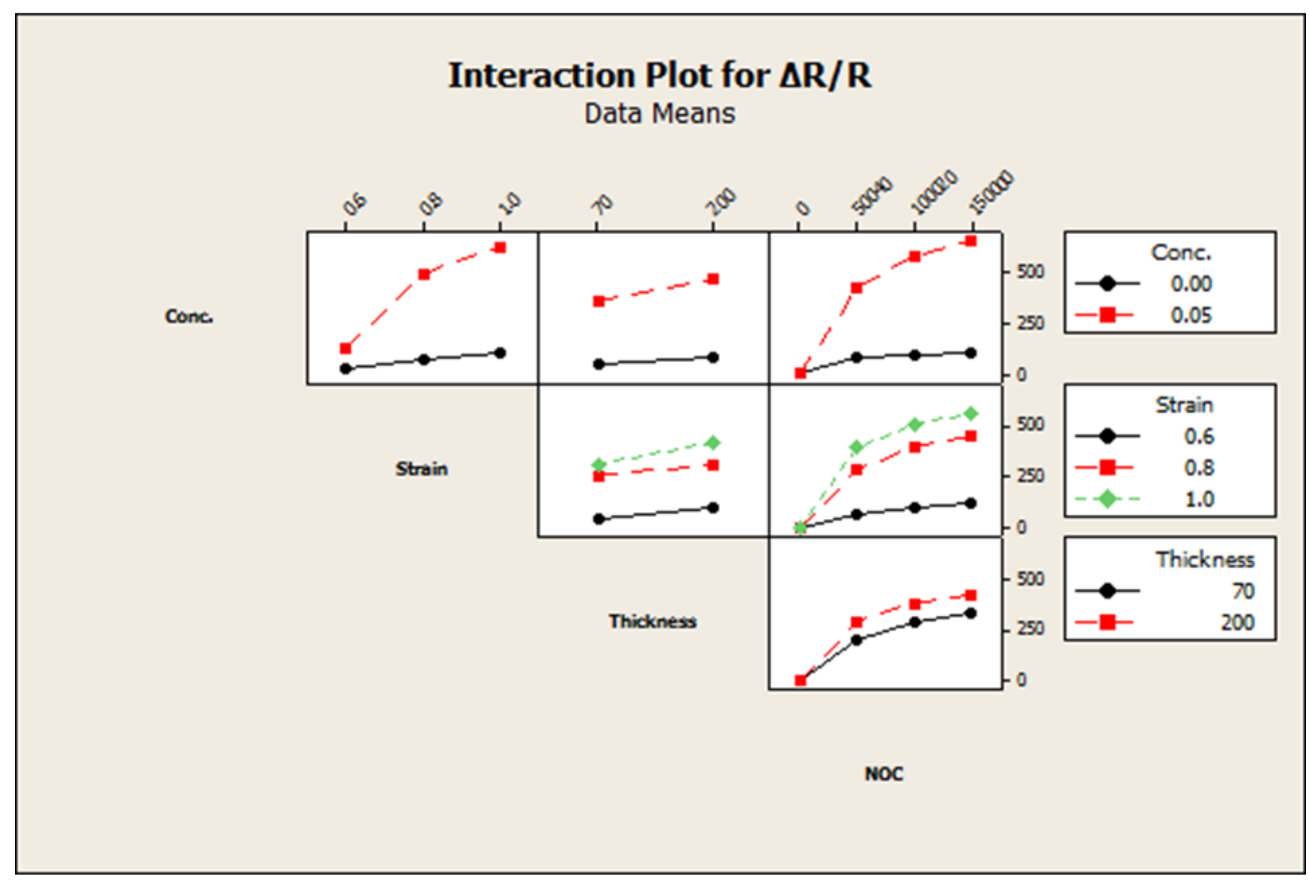

Fig. 5.7 Interaction plot for change in electrical resistance for ITO films under fatigue and fatigue-corrosion. 
The ANOVA for bending and bending-corrosion experiments is shown in Table 5.2. All design factors and their two-way interactions except a two-way interaction of thickness and time are observed to be significant. However, only one three-way interaction involving thickness is observed to be significant.

Table 5.2 Analysis of variance (ANOVA) for bending and bending-corrosion

\begin{tabular}{|lrrrrrr|}
\hline Source & DF & Seq SS & Adj SS & Adj MS & F & P \\
\hline Conc. & 1 & 3128.66 & 3128.66 & 3128.66 & 497.66 & 0.0000 \\
Strain & 2 & 1190.76 & 1190.76 & 595.38 & 94.7 & 0.0000 \\
Thickness & 1 & 76.27 & 76.27 & 76.27 & 12.13 & 0.0130 \\
Time & 3 & 2185.73 & 2185.73 & 728.58 & 115.89 & 0.0000 \\
Conc.*Strain & 2 & 781.43 & 781.43 & 390.72 & 62.15 & 0.0000 \\
Conc.*Thickness & 1 & 42.5 & 42.5 & 42.5 & 6.76 & 0.0410 \\
Conc.*Time & 3 & 1111.75 & 1111.75 & 370.58 & 58.95 & 0.0000 \\
Strain*Thickness & 2 & 89.06 & 89.06 & 44.53 & 7.08 & 0.0260 \\
Strain*Time & 6 & 419.32 & 419.32 & 69.89 & 11.12 & 0.0050 \\
Thickness*Time & 3 & 37.76 & 37.76 & 12.59 & 2 & 0.2150 \\
Conc.*Strain*Thickness & 2 & 75.27 & 75.27 & 37.63 & 5.99 & 0.0370 \\
Conc.*Strain*Time & 6 & 279.77 & 279.77 & 46.63 & 7.42 & 0.0140 \\
Conc.*Thickness*Time & 3 & 24.12 & 24.12 & 8.04 & 1.28 & 0.3640 \\
Strain*Thickness*Time & 6 & 43.18 & 43.18 & 7.2 & 1.14 & 0.4370 \\
Error & 6 & 37.72 & 37.72 & 6.29 & & \\
Total & 47 & 9523.3 & & & & \\
S= 2.50735 & R-sq=99.60\% & R-sq(adj) $=\mathbf{9 6 . 9 0 \%}$ & & & \\
\hline
\end{tabular}

In addition, comparable trends of main effects and two-way interactions are observed for bending and bending-corrosion as in fatigue and fatigue-corrosion as shown in Figs. 5.8 and 5.9 respectively. However, the maximum change in electrical resistance under bending-corrosion is $59 \%$ whereas under fatigue-corrosion it is observed to be $1107 \%$. This further confirms the longterm significance of fatigue-corrosion in the design of optoelectronic devices. 


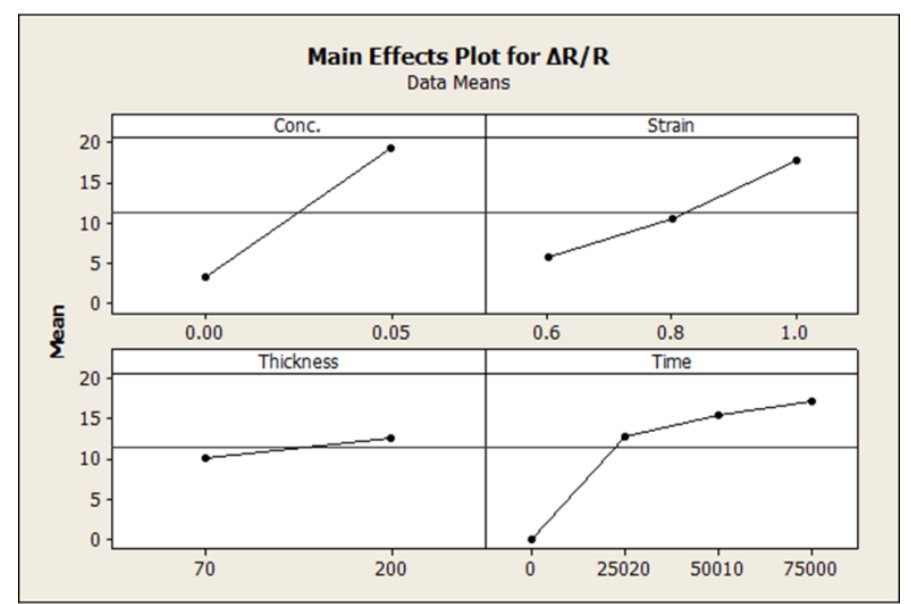

Fig. 5.8 Main effects plot for change in electrical resistance for ITO films under bending and bending-corrosion.

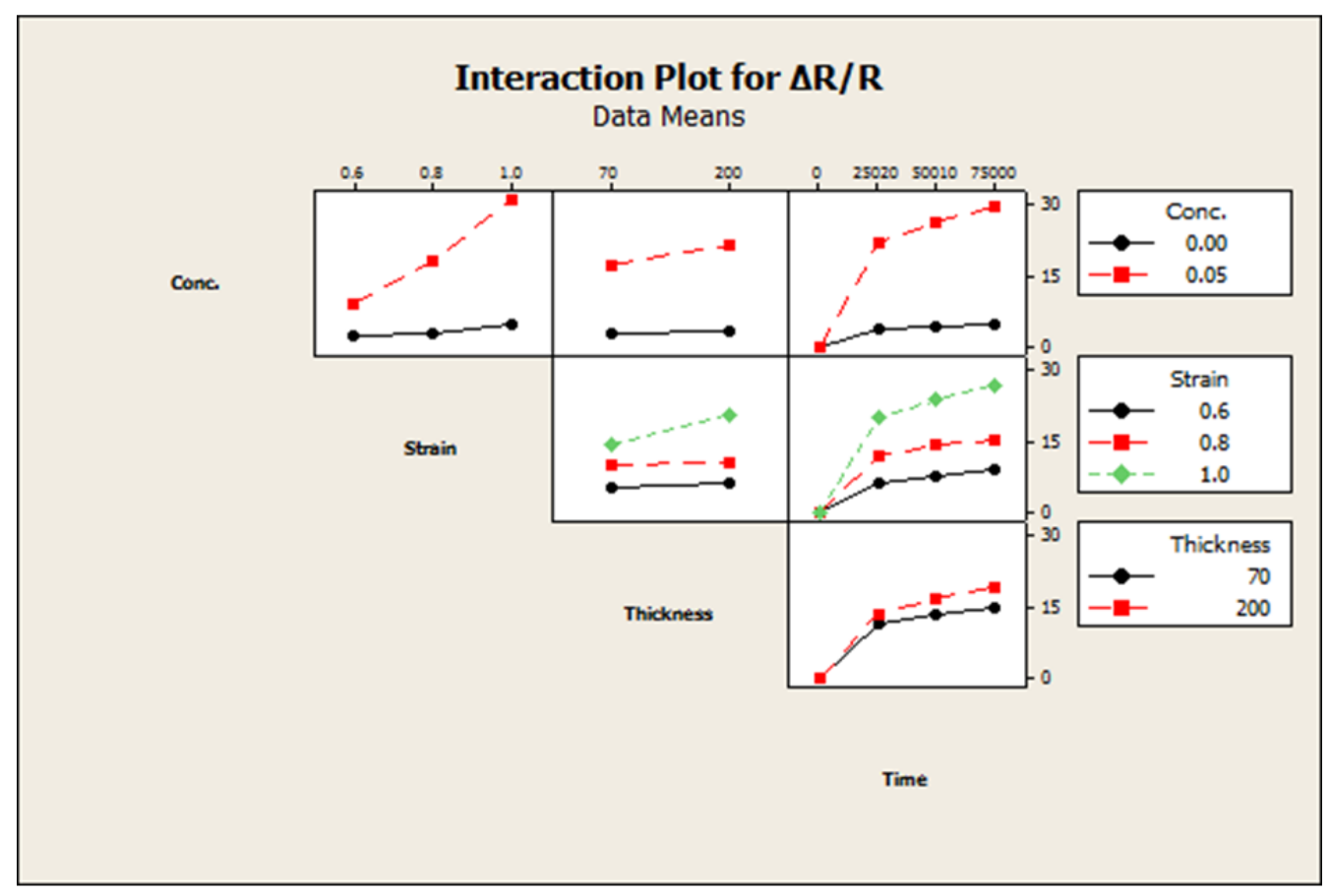

Fig. 5.9 Interaction plot for change in electrical resistance for ITO films under bending and bending-corrosion. 
The reliability of the ANOVA results is confirmed by the normal probability plots of the randomly distributed residuals around the mean as shown in Fig. 5.10.
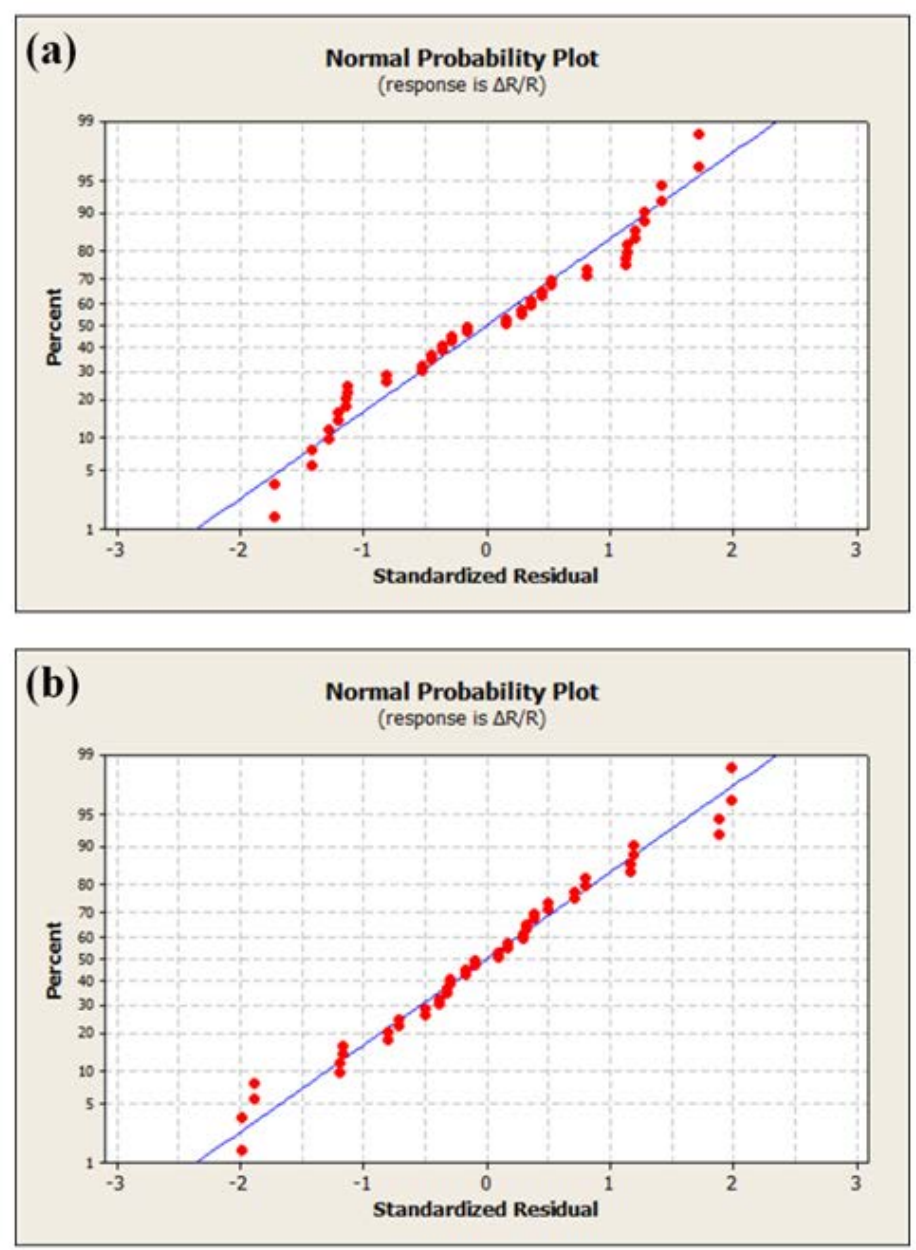

Fig. 5.10 Normal probability plot for change in electrical resistance for ITO films under: (a) fatigue and fatigue-corrosion (b) bending and bending-corrosion.

The equations of the regression models to predict the significant effects of the factors and their interactions on the change in electrical resistance under fatigue-corrosion (Eq. 5.1) and static bending-corrosion (Eq. 5.2) are given below. 


$$
\begin{gathered}
\Delta R / R, \%=427-17099 A C-0.0056 N O C+21311 A C * S+0.0736 A C * N O C+0.00701 S \\
* N O C \\
\begin{aligned}
\Delta R / R, \%= & 26.6-778 A C-29.7 S-0.000458 T+985 A C * S+0.00625 A C * T \\
+ & 0.000567 S * T
\end{aligned}
\end{gathered}
$$

where AC, S, NOC, and T, are acid concentration, applied strain, number of cycles, and time, respectively. The terms of p-values (Table 5.3 and 5.4) greater than 0.05 are removed from the regression model since they are not statistically significant.

Table 5.3 Regression coefficients for fatigue and fatigue-corrosion

\begin{tabular}{|lrrrc|}
\hline Predictor & \multicolumn{1}{c}{ Coef } & SE Coef & \multicolumn{1}{c|}{ T } & P \\
\hline Constant & 427.2 & 251.7 & 1.7 & 0.098 \\
Conc. & -17099 & 3814 & -4.48 & 0.000 \\
Strain & -491.9 & 298.2 & -1.65 & 0.108 \\
Thickness & -1.066 & 1.387 & -0.77 & 0.447 \\
NOC & -0.0056 & 0.001684 & -3.33 & 0.002 \\
Conc.*Strain & 21311 & 4187 & 5.09 & 0.000 \\
Conc.*Thickness & 11.35 & 10.52 & 1.08 & 0.288 \\
Conc.*NOC & 0.07357 & 0.01223 & 6.01 & 0.000 \\
Strain*Thickness & 1.215 & 1.611 & 0.75 & 0.455 \\
Strain*NOC & 0.007014 & 0.001873 & 3.75 & 0.001 \\
Thickness*NOC & 0.00000462 & 0.0000047 & 0.98 & 0.333 \\
S = 118.437 R-sq $=\mathbf{8 9 . 1 \%}$ & $\mathbf{R - s q}(\mathbf{a d j})=\mathbf{8 6 . 1 \%}$ & & \\
\hline
\end{tabular}


Table 5.4 Regression coefficients for bending and bending-corrosion

\begin{tabular}{|lrrrc|}
\hline Predictor & \multicolumn{1}{c}{ Coef } & SE Coef & \multicolumn{1}{c|}{ T } & P \\
\hline Constant & 26.55 & 12.19 & 2.18 & 0.036 \\
Conc. & -777.9 & 184.8 & -4.21 & 0.000 \\
Strain & -29.72 & 14.45 & -2.06 & 0.047 \\
Thickness & -0.09684 & 0.06719 & -1.44 & 0.158 \\
Time & -0.0004577 & 0.0001632 & -2.81 & 0.008 \\
Conc.*Strain & 985.4 & 202.9 & 4.86 & 0.000 \\
Conc.*Thickness & 0.579 & 0.5097 & 1.14 & 0.263 \\
Conc.*Time & 0.006247 & 0.001185 & 5.27 & 0.000 \\
Strain*Thickness & 0.10439 & 0.07803 & 1.34 & 0.189 \\
Strain*Time & 0.0005668 & 0.0001815 & 3.12 & 0.003 \\
Thickness*Time & 0.00000049 & 0.00000046 & 1.07 & 0.293 \\
S= 5.73835 $\quad$ R-sq $=\mathbf{8 7 . 2 \%}$ & $\mathbf{R}-\mathbf{s q}(\mathbf{a d j})=\mathbf{8 3 . 7} \%$ & & \\
\hline
\end{tabular}

\subsubsection{Microscopy}

Figs. 5.11 and 5.12 show a typical ITO film cracking progression under fatigue and fatigue-corrosion degradation. When the number of cycles reaches a critical value, straight line cracks perpendicular to the straining direction is observed as shown in Figs. 5.11a and 5.12a. As the number of cycles increases, more cracks are observed to form. This is also confirmed by the crack density versus NOC relation shown in Fig. 5.13. However, the crack morphology is different for fatigue and fatigue-corrosion experiments. In particular, secondary cracks in the transverse direction are observed to form at a higher NOC for specimens under fatigue-corrosion as shown in Figs. 5.12e and f. Fatigue striations due to an increment of crack growth and simultaneous blunting and resharpening of the crack tip by plastic deformation are observed [126]. Furthermore, coating delamination is observed (Fig. 5.12f) for films under fatiguecorrosion at 150,000 cycles. 


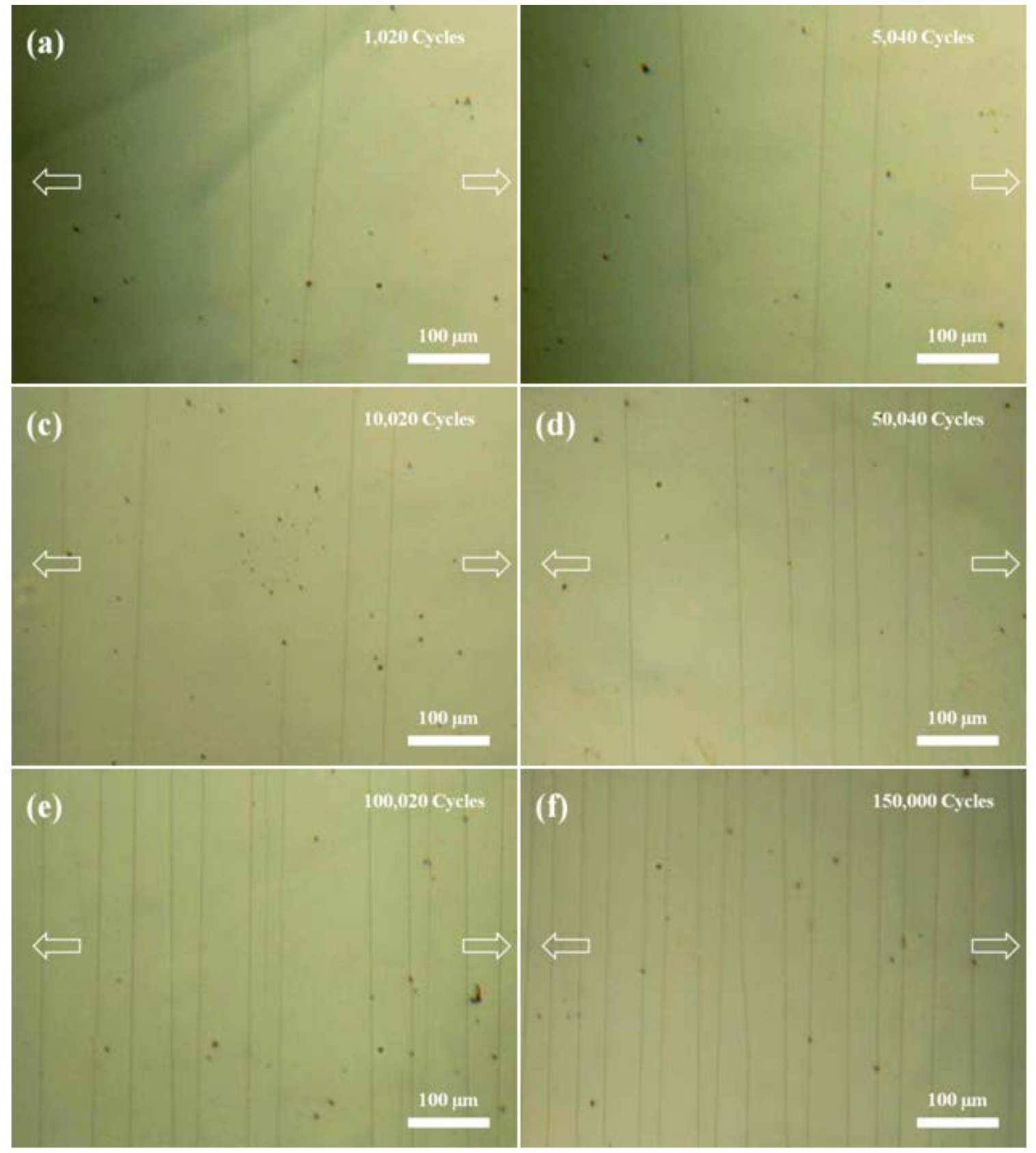

Fig. 5.11 Optical microscopy images of cracked $200 \mathrm{~nm}$ thick ITO films under fatigue. The corresponding number of cycle values is indicated on the images. Arrows indicate the tensile direction. Applied strain is $1.0 \%$. 


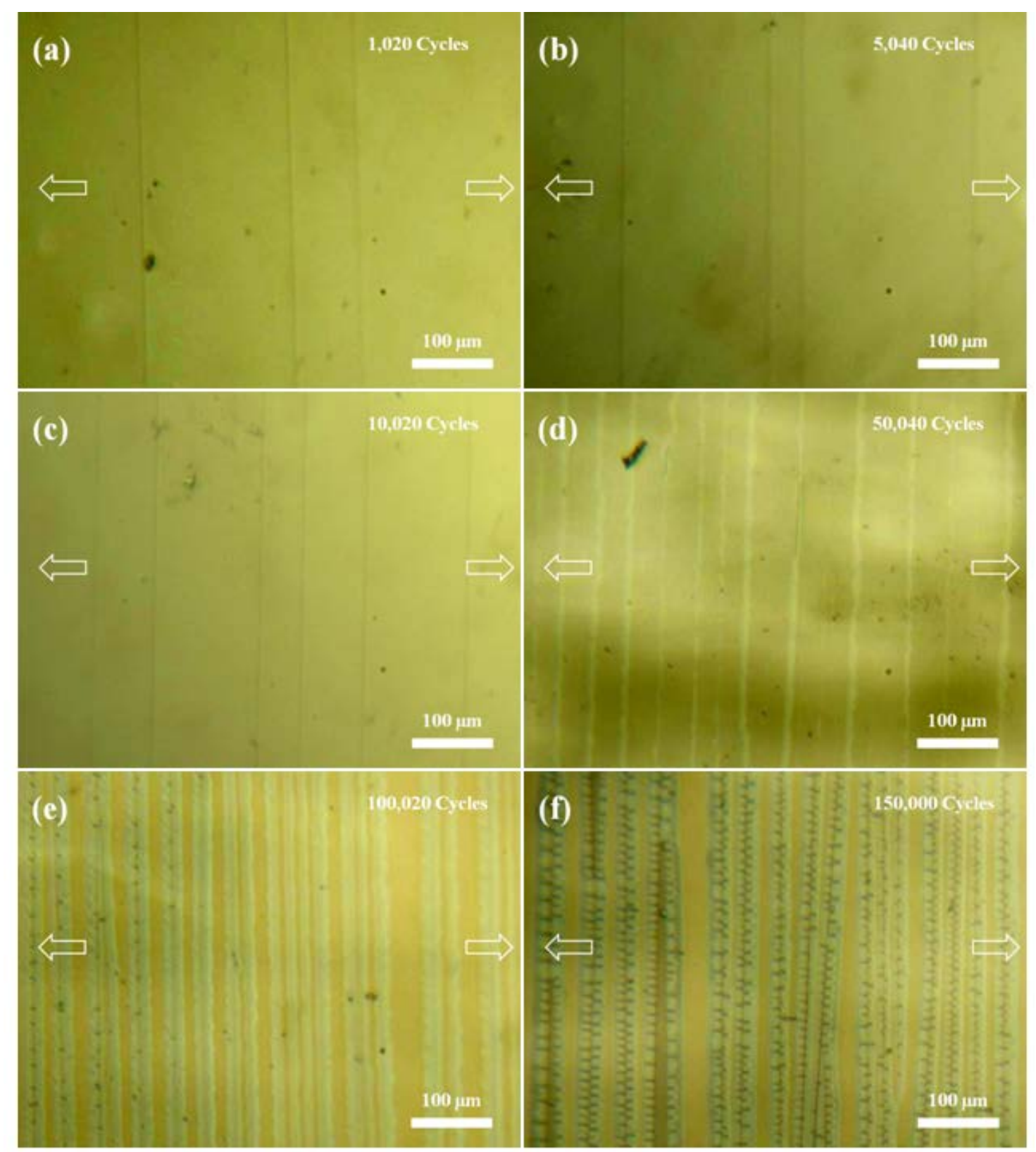

Fig. 5.12 Optical microscopy images of cracked $200 \mathrm{~nm}$ thick ITO films under fatigue-corrosion. The corresponding number of cycle values is indicated on the images. Arrows indicate the tensile direction. Applied strain is $1.0 \%$.

Figs. 5.14 and 5.15 show an SEM image of ITO and CNT-based conducting surfaces under fatigue-corrosion experiments, respectively. Severe coating delamination is observed for 
the ITO surface after 150,000 cycles of testing. On the other hand under the same conditions, very limited binder delamination is observed with the underlying CNTs being mostly intact. This is in agreement with the electrical resistance changes observed for both materials.

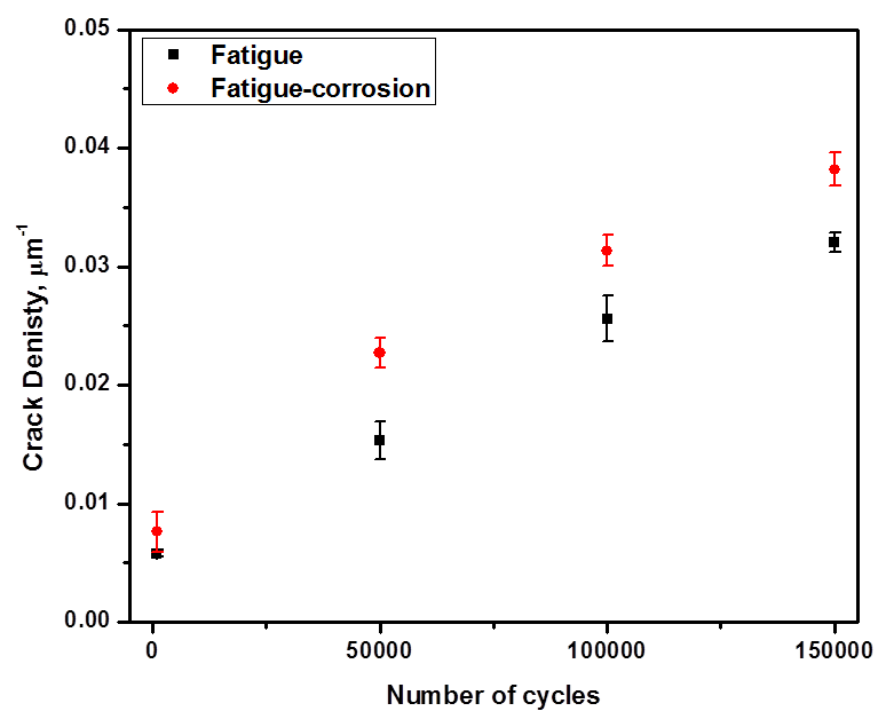

Fig. 5.13 Crack density versus number of cycles for $200 \mathrm{~nm}$ thick ITO films under fatigue and fatigue-corrosion.

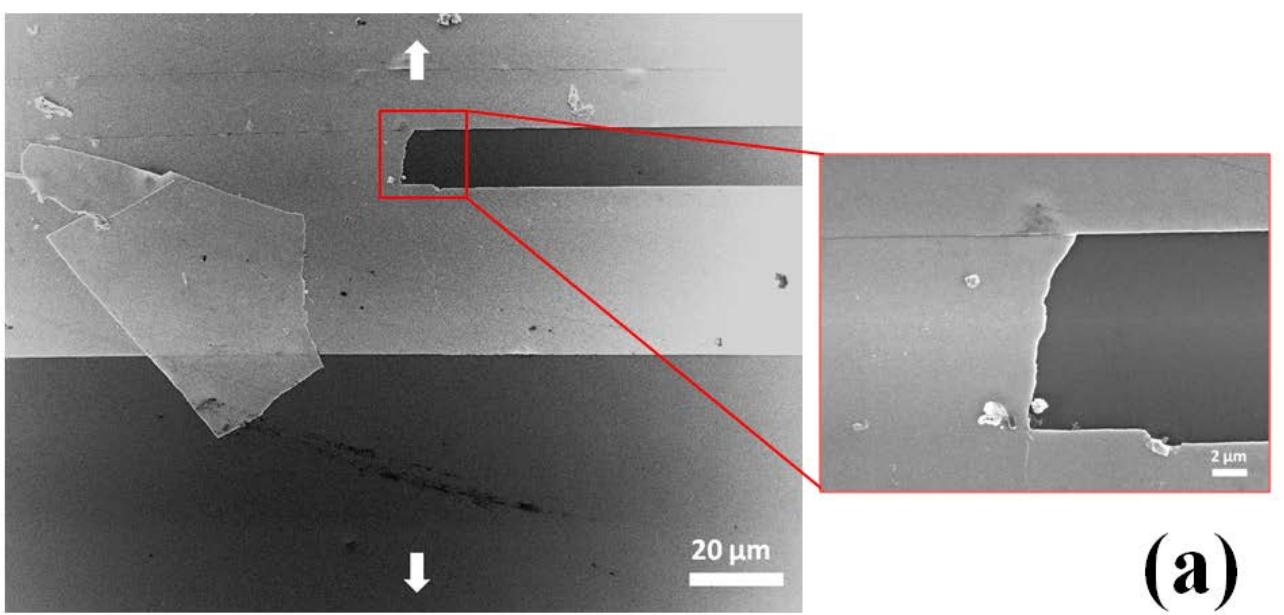



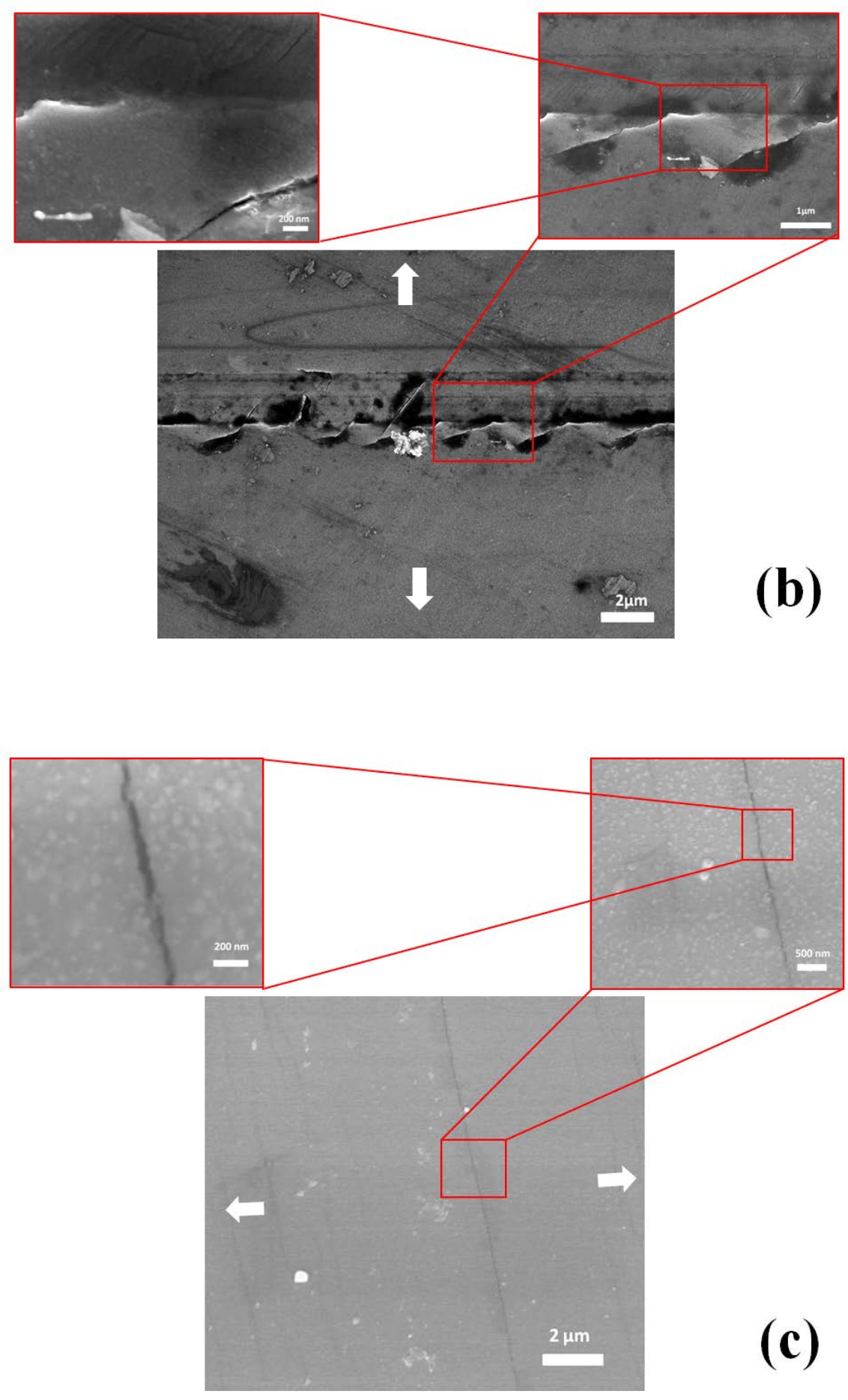

Fig. 5.14 SEM image showing cracks on the surface ITO coated PET at $1.0 \%$ applied strain and 150,000 cycles under fatigue-corrosion. Arrows indicate the tensile direction. 


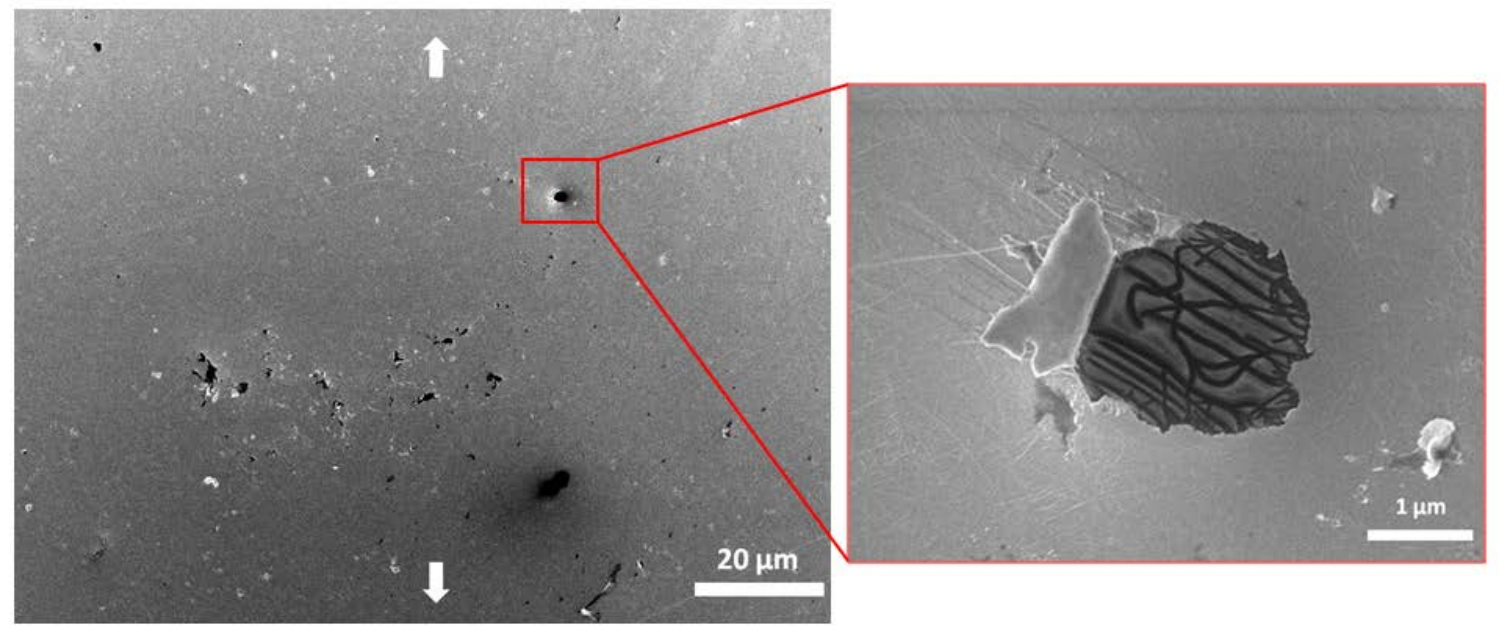

Fig. 5.15 SEM image showing delamination of top coat on the surface CNT coated PET at $1.0 \%$ applied strain and 150,000 cycles under fatigue-corrosion. Arrows indicate the tensile direction.

\subsubsection{Finite Element Analysis}

Fig. 5.16 shows the Von Mises stress distribution. We observe that the highest stress is located in the middle section of the top ITO layer. This is indicating a higher potential for crack initiation under fatigue conditions. This is a typical and therefore expected response of thin films on compliant substrates [127]. The stress distribution, for the three different bending mandrels (Fig. 5.17a) and for the two different film thicknesses (Fig. 5.17b), indicates that the effect of applied strain is more significant than the film thickness factor. This is in agreement with our DOE results. 


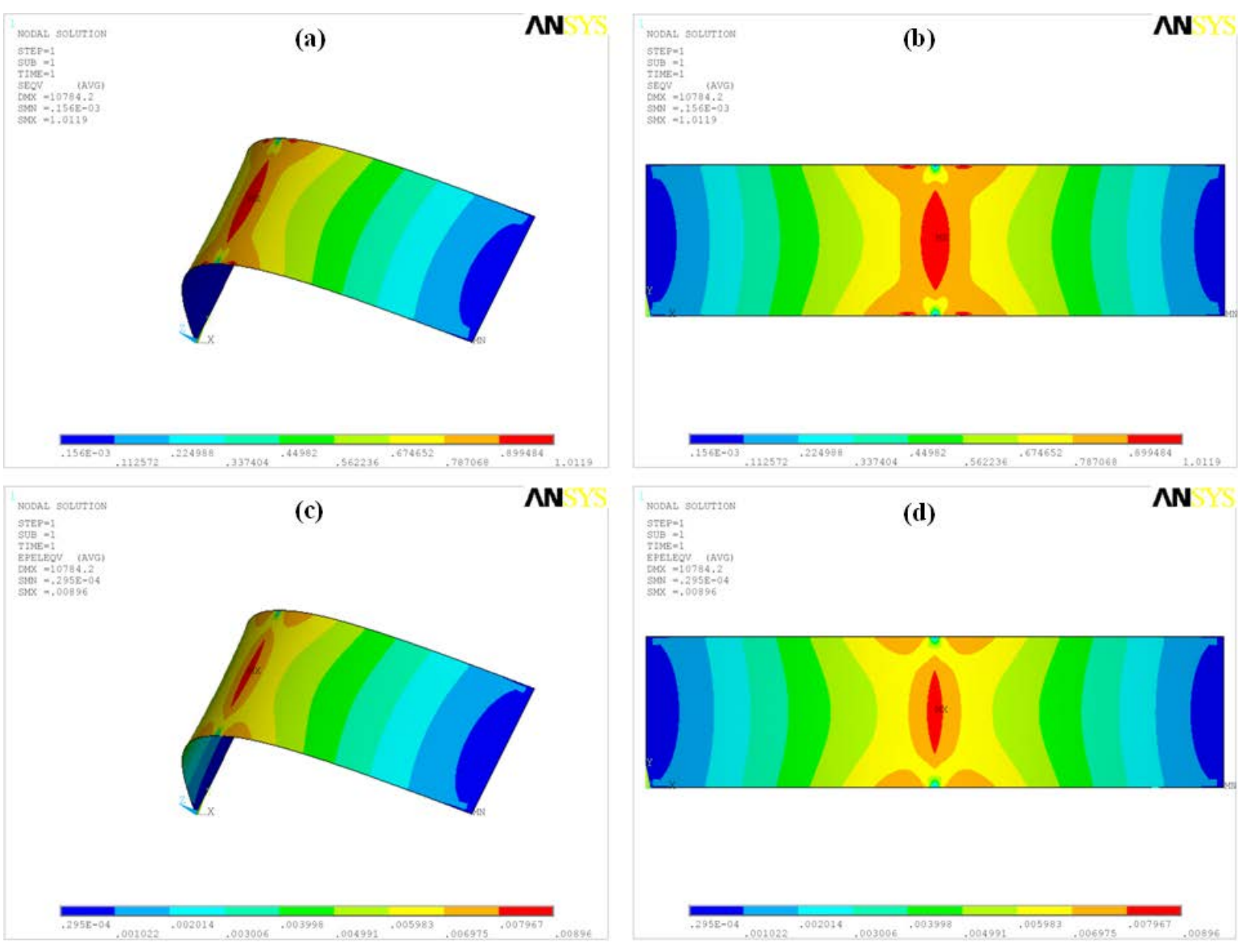

Fig. 5.16 (a) and (b) Von Mises stress distribution, and (c) and (d) Von Mises strain distribution of $200 \mathrm{~nm}$ thick ITO-coated PET. 
(a)

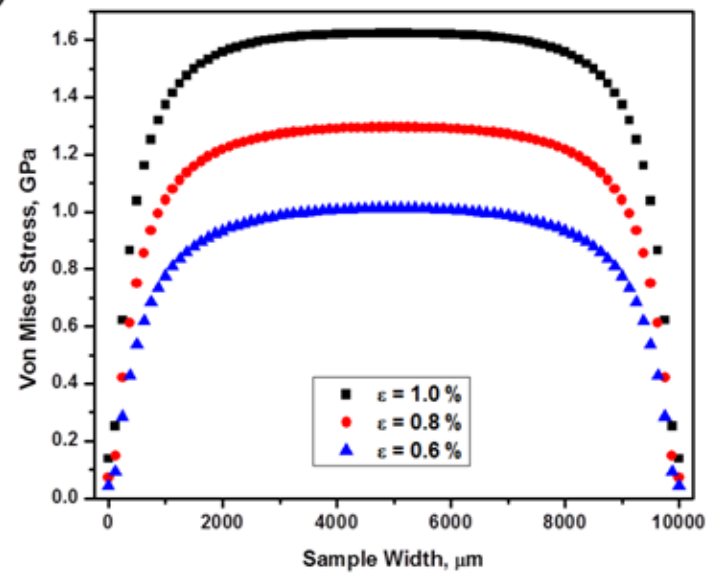

(b)

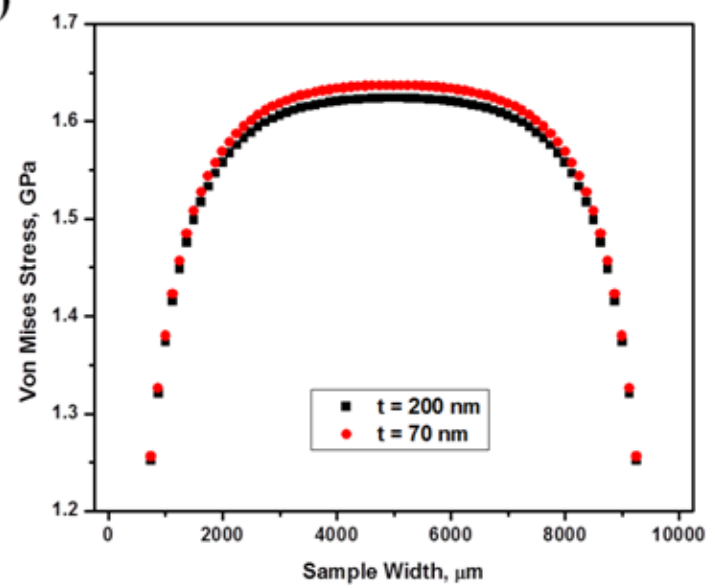

Fig. 5.17 (a) Effect of applied strain and (b) effect of thickness on the stress distribution of ITO film.

\subsubsection{Life-stress model}

Life prediction of ITO films are performed for both fatigue and fatigue-corrosion experiments. Cycles to failure data obtained from accelerated electromechanical testing and stress values from FEA were analyzed using IPL-Weibull model with maximum likelihood estimation method. Fig. 5.18 shows a typical relation of cycles to failure versus mechanical 
stress under fatigue and fatigue-corrosion conditions. The parameters of the assumed model for both fatigue and fatigue-corrosion conditions are summarized in Table 5.5. The parameter $n$ in the inverse power relationship (Eq. 3.5) is a measure of the effect of the stress on the cycles to failure [128]. Thus, the higher n values observed for the thicker films, under both experimental conditions, suggest that they are more sensitive to stress variation and thus, lower cycles to failure values than their thinner counterparts.
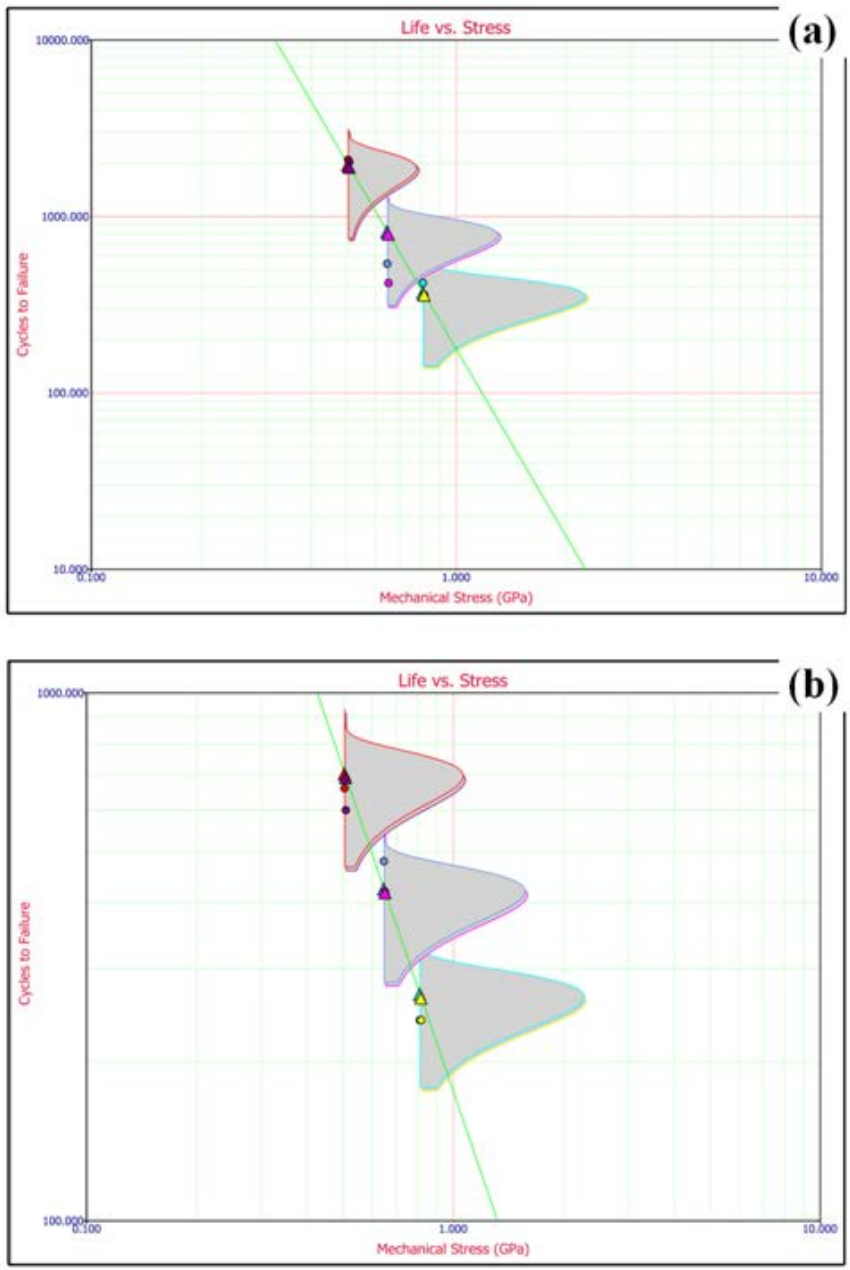

Fig. 5.18 Cycles to failure versus stress for the $200 \mathrm{~nm}$ thick ITO film under: (a) fatigue and (b) fatigue-corrosion. 
Table 5.5 Parameters for IPL-Weibull model

\begin{tabular}{|ccccc|}
\hline \multirow{3}{*}{ Parameter } & \multicolumn{2}{c}{ Fatigue } & \multicolumn{2}{c|}{ Fatigue-corrosion } \\
\cline { 2 - 5 } & \multicolumn{4}{c}{ Film thickness } \\
\cline { 2 - 5 } & $70 \mathrm{~nm}$ & $200 \mathrm{~nm}$ & $70 \mathrm{~nm}$ & $200 \mathrm{~nm}$ \\
\hline$\beta$ & 10.6781 & 5.1434 & 14.3809 & 10.3278 \\
$\mathrm{~K}$ & 0.0039 & 0.0057 & 0.0030 & 0.0057 \\
$\mathrm{n}$ & 3.2366 & 3.5289 & 1.1406 & 2.0348 \\
\hline
\end{tabular}




\section{CONTROLLED-BUCKLING BEHAVIOR OF PATTERNED ELECTRODES}

\subsection{Introduction}

In this chapter the mechanical behavior of various patterned ITO shapes and sizes on PET was investigated. Micron-sized shapes include squares, circles, and zigzag-based structures. Controlled-buckling experiments were performed in-situ using an optical microscope in order to monitor critical strains and potential failure mechanisms. In addition, ITO electrical resistance changes were continuously monitored during deformation. Furthermore, ex-situ characterization of the tested surfaces using scanning electron microscopy was conducted. Patterned brittle structures may be able to accommodate higher mechanical strains than their full film covered counterparts because of their controlled shape and size.

\subsection{Results and discussion}

\subsubsection{Finite element analysis results}

The Von Mises stress distribution for both the unpatterned and patterned films under buckling conditions is shown in Fig. 6.1. In both ITO tensile and compressive buckling modes, the highest stress is found to be concentrated around the central part and at the clamped edges of the top ITO layer. On the other hand, the lowest stress is observed to occur at the PET substrate. This bell shaped stress distribution is typical for relatively high modulus coatings on compliant substrates 
[127] and it is in agreement with experimental results since cracks are observed to initiate at such locations.
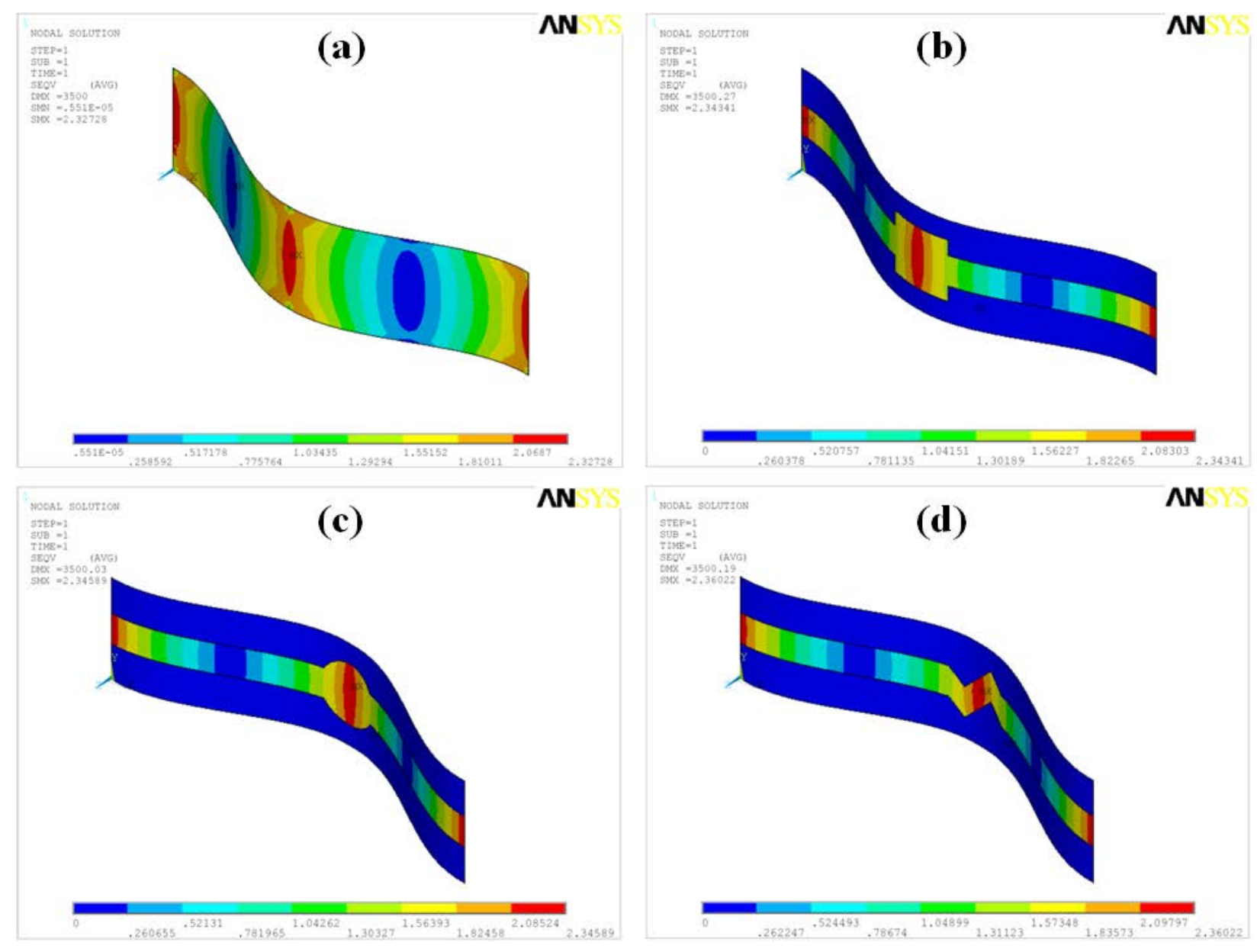

Fig. 6.1 Von Mises stress distribution of (a) unpatterned and (b) square-patterned film under tensile buckling; (c) circle and (d) zigzag patterned film under compressive buckling.

The maximum Von Misses stress values for square, circle, and zigzag-patterned ITO films were found to be 2.343, 2.346, and $2.360 \mathrm{GPa}$, respectively. The highest stress is obtained for the zigzag-patterned film while the lowest stress is observed for the square-patterned film. This can be due to the smaller material volume present in the zigzag patterned film as illustrated in Table 
6.1. However, the results of the model can only be taken as indicative since a film of uniform thickness, without any defect, and no edge-effects was considered. In practice, the edge definition of the etched surfaces may play an important role in the mechanical behavior of the buckled structure.

Table 6.1 Calculated volume for different patterned shapes of ITO and their corresponding values of (a) COS-R, (b) COS-M, and (c) $\Delta R / R$ at COS-M. $A_{1} / A_{2}$ is the change in area along the axis of the patterns.

\begin{tabular}{|c|c|c|c|c|c|c|c|}
\hline \multicolumn{2}{|c|}{ Size } & \multicolumn{3}{|c|}{ Small } & \multicolumn{3}{|c|}{ Large } \\
\hline \multicolumn{2}{|c|}{ Shape } & Square & Circle & Zigzag & Square & Circle & Zigzag \\
\hline \multicolumn{2}{|c|}{ Volume $\left(\mu \mathrm{m}^{3}\right)$} & 7000 & 5955.32 & 4200 & 35000 & 29776.6 & 21000 \\
\hline \multicolumn{2}{|c|}{$\mathrm{A}_{1} / \mathrm{A}_{2}$} & 2 & 1 & 1 & 2 & 1 & 1 \\
\hline \multirow{2}{*}{ Sharpness } & Angle & 90 & 119 & 90 & 90 & 119 & 90 \\
\hline & Corners & 4 & 4 & 1 & 4 & 4 & 1 \\
\hline \multirow{2}{*}{ 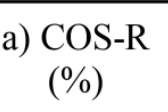 } & Tensile & 0.977 & 1.379 & 1.286 & 0.919 & 1.194 & 1.026 \\
\hline & Compressive & 1.3362 & 1.8463 & 1.721 & 1.332 & 1.552 & 1.375 \\
\hline \multirow{2}{*}{$\begin{array}{l}\text { b) COS- } \\
\text { M (\%) }\end{array}$} & Tensile & 1.563 & 1.741 & 1.731 & 1.529 & 1.624 & 1.476 \\
\hline & Compressive & 1.577 & 1.921 & 1.87 & 1.64 & 1.626 & 1.554 \\
\hline \multirow{2}{*}{$\begin{array}{l}\text { c) } \Delta \mathrm{R} / \mathrm{R} \\
\text { at COS- } \\
\mathrm{M}(\%)\end{array}$} & Tensile & 44.7215 & 25.199 & 19.13 & 55.2025 & 39.394 & 34.121 \\
\hline & Compressive & 25.964 & 13.559 & 12.15 & 31.4045 & 21.193 & 18.1895 \\
\hline
\end{tabular}

\subsubsection{Controlled buckling experiments}

In order to investigate the effect of size and shape of different patterns of ITO coated PET films, specimens were strained up to a $6 \%$ applied strain under controlled buckling conditions. During testing, the ITO surface was placed under both tension and compression with in-situ electrical resistance and surface optical microscopy monitoring. The crack onset strain was 
determined using both the normalized electrical resistance (COS-R) and optical microscopy (COS-M) monitoring tools. COS-R values were determined from the sudden increase in electrical resistance (Figs. 6.2 and 6.3). In particular, an increase of 10\% in electrical resistance is chosen arbitrarily to denote the crack onset initiation, COS-R [69].
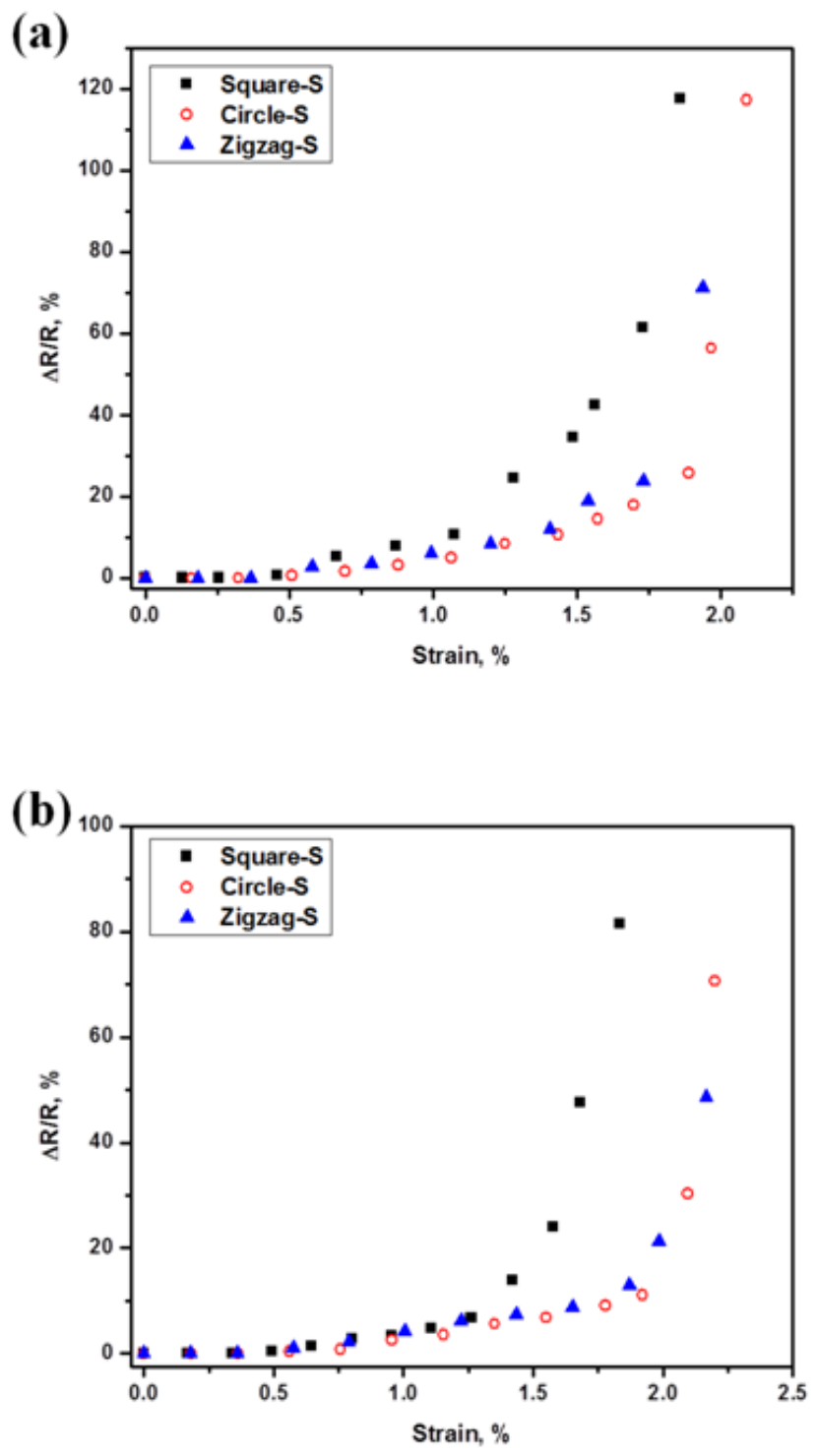

Fig. 6.2 Normalized electrical resistance versus applied strain for the smaller size patterned ITO coated PET films under (a) tension and (b) compression. 

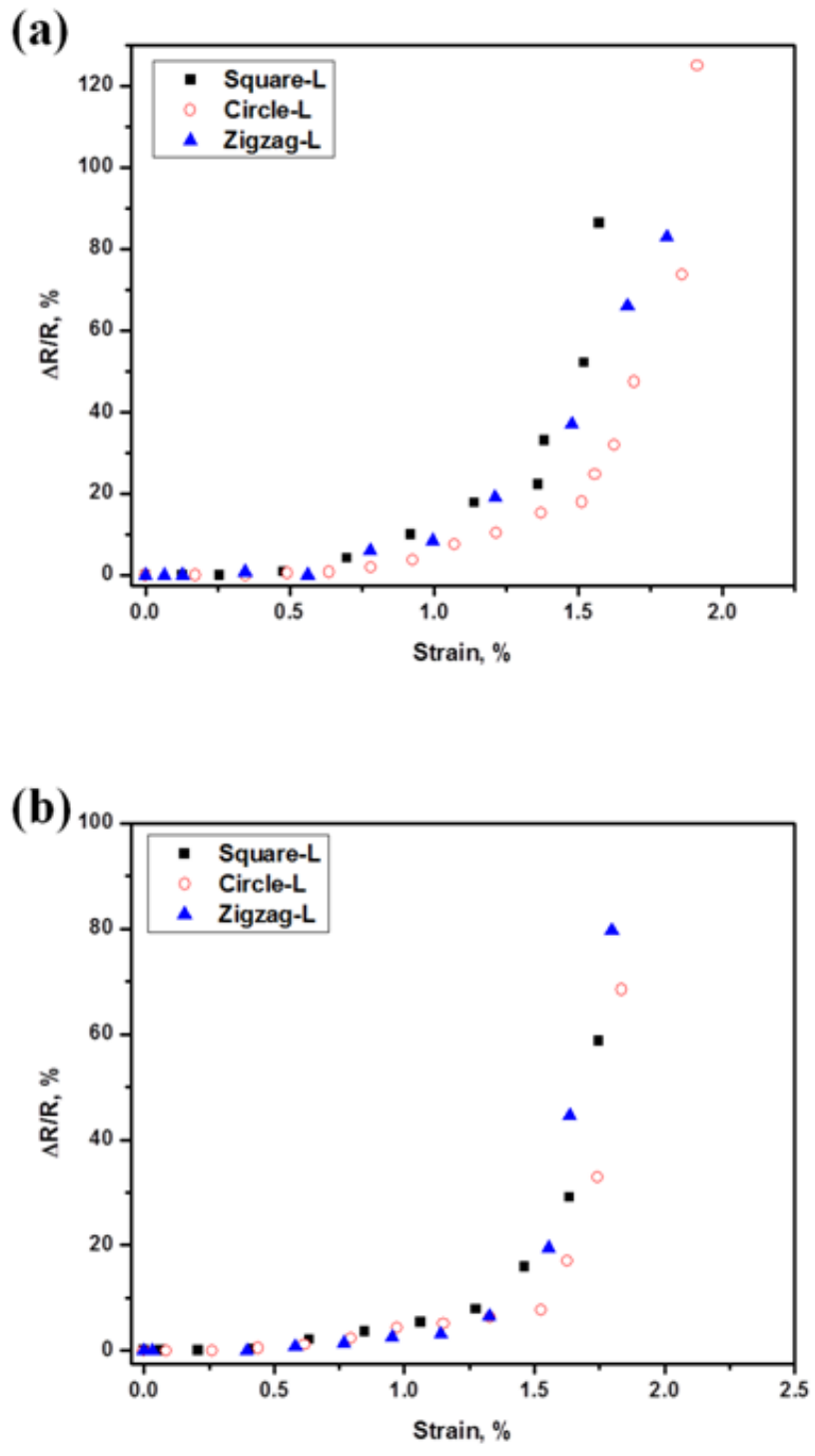

Fig. 6.3 Normalized electrical resistance versus applied strain for the larger size patterned ITO coated PET films under (a) tension and (b) compression.

Fig. 6.2 and 6.3 show a typical change in ITO electrical resistance for layers under both tensile (Fig. 6.2a and 6.3a) and compressive (Fig. 6.2b and 6.3b) buckling modes. A slightly more rapid increase in resistance is observed in the case of the smaller size square-shaped 
patterns, around $1.25 \%$ tensile strain and $1.5 \%$ compressive strain respectively. The COS-R values under tension and compression are summarized in Fig. 6.4. They indicate that the tensile mode is more critical than the compressive mode. In particular, the tensile COS-R value for the smaller size circular pattern is observed to be $1.379 \%$ whereas the compressive COR-R value is equal to $1.846 \%$. These COS-R values are comparable with unpatterned ITO films on PET substrates [75]. In addition, the higher COS-R values, both under tension and compression, observed for the smaller size patterns may suggest that a reduced volume of active brittle material leads to improved reliability due to the reduced number of intrinsic defects present in the film. The effects of material volume on the mechanical behavior of thin films have been discussed previously. As the volume of the material increases, lower values of yield stress were obtained [129].

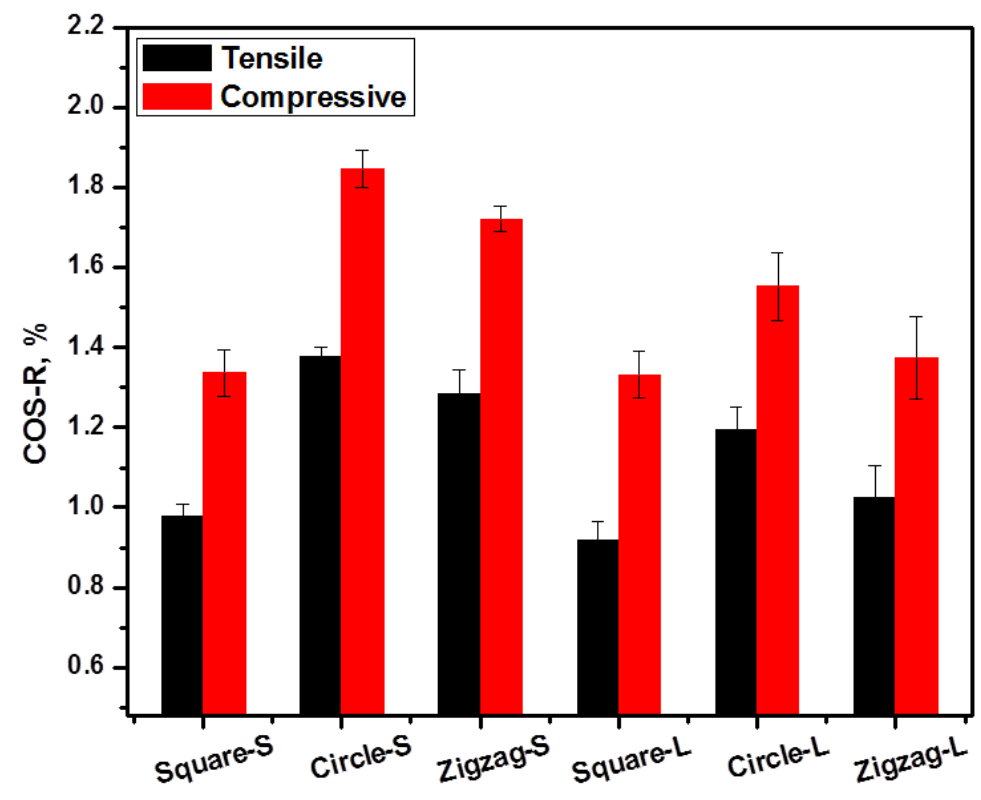

Fig. 6.4 Crack onset strain from change in electrical resistance monitoring of ITO coated PET films. 
Furthermore, the COS-R values indicate that the smaller size circular and zigzag patterned ITO films performed better than the square patterned films. In addition to the volume differences, this can be associated with the change in area $\left(A_{1} / A_{2}\right.$ is the change in area along the axis of the pattern), sharpness, and number of corners present in the patterns. Fig. 3.1b shows the sharpness angle, and Table 6.1a illustrates calculated volume and the corresponding COS-R values. Considering small size patterns, the square-shaped pattern exhibits the highest combination of material volume, change in area $\left(\mathrm{A}_{1} / \mathrm{A}_{2}\right)$, corner angle, and number of corners. It is believed that such combination contributes to the stress concentration factor which may initiate cracks in the brittle coating earlier as compared to the other patterned film structures.

\subsubsection{Microscopy}

Fig. 6.5 and 6.6 show a typical cohesive failure progression under tensile and compressive buckling deformation modes, respectively. When the externally applied strain reaches a critical value, the onset of straight line cracks oriented perpendicular to the compression direction is observed (Fig. 6.5b and 6.6b). Initially very few cracks are forming. However, as the externally applied strain increases (Fig. 6.5c and 6.6c), more cracks are observed to form. Further increase of applied strain, as depicted in Fig. 6.5d and 6.6d, results in an increased number of cracks and an associated decrease in spacing between them. The contraction of the PET substrate underneath the coating cracked edges shown in Fig. 6.6d indicates coating delamination due to the applied compressive mode. Similar cohesive failure progression is observed for both square and zigzag-shaped patterns as shown in Fig. 6.7 and 6.8 
and Fig. 6.9 and 6.10, respectively. The coating delamination of specimens under compressive buckling failure mode is confirmed by the SEM micrographs shown in Fig. 7.11b. This is a typical buckling-driven delamination case for ITO films on polymer substrates [78, 130]. Fig. 7.11a shows a typical cohesive cracking failure for specimens under tensile buckling mode. In both cases, it is observed that failure is driven by micro-cracks initiating from the edges. Controlling the edge etch definition is of paramount importance and can lead to increased reliability of the patterned coatings since it will diminish the edge-generated micro-cracks.
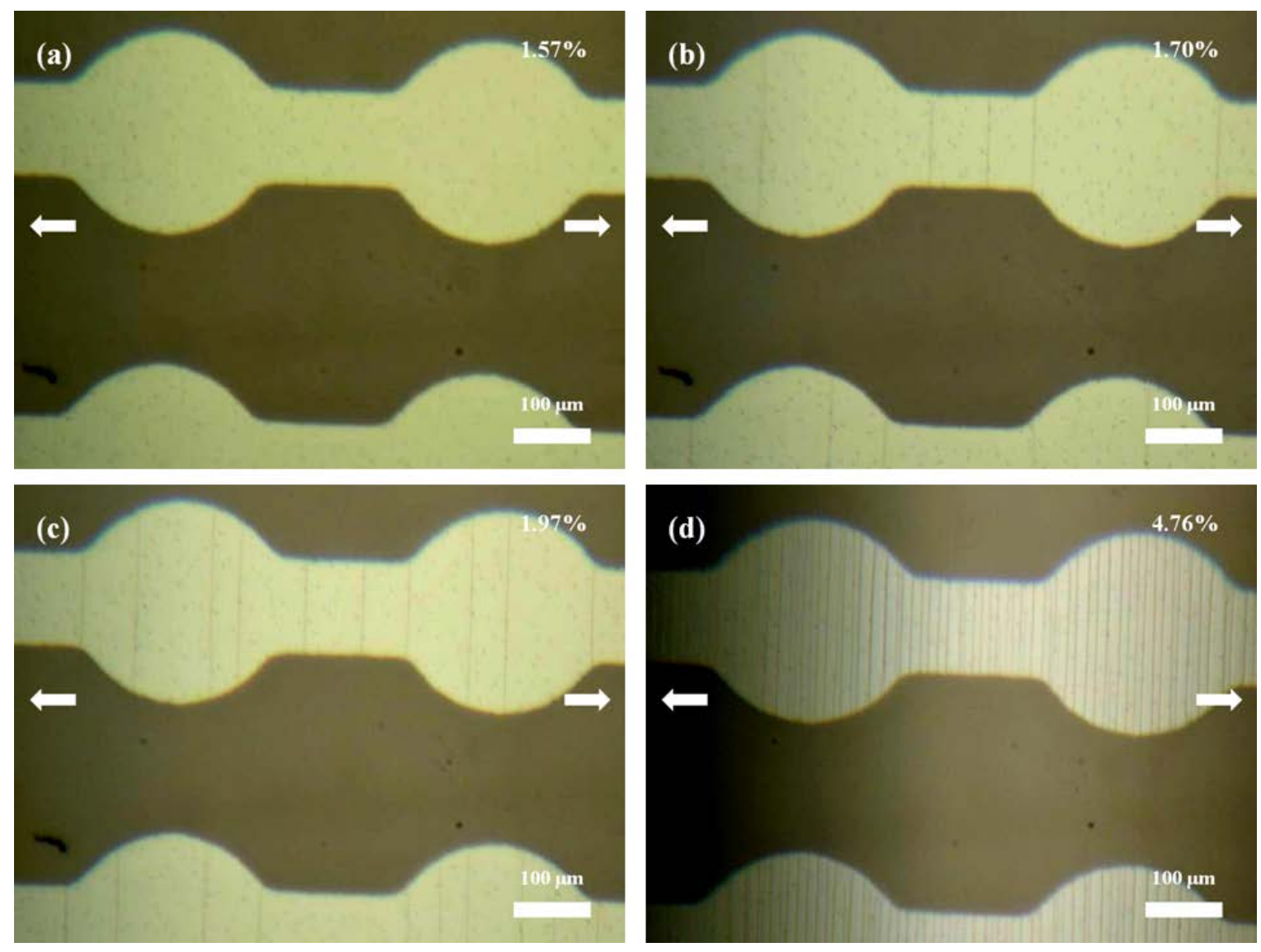

Fig. 6.5 Optical microscopy images of circular-patterned ITO coated PET films under tensile buckling deformation mode showing crack initiation and propagation up to $6 \%$ strain. Arrows indicate the buckling direction. 

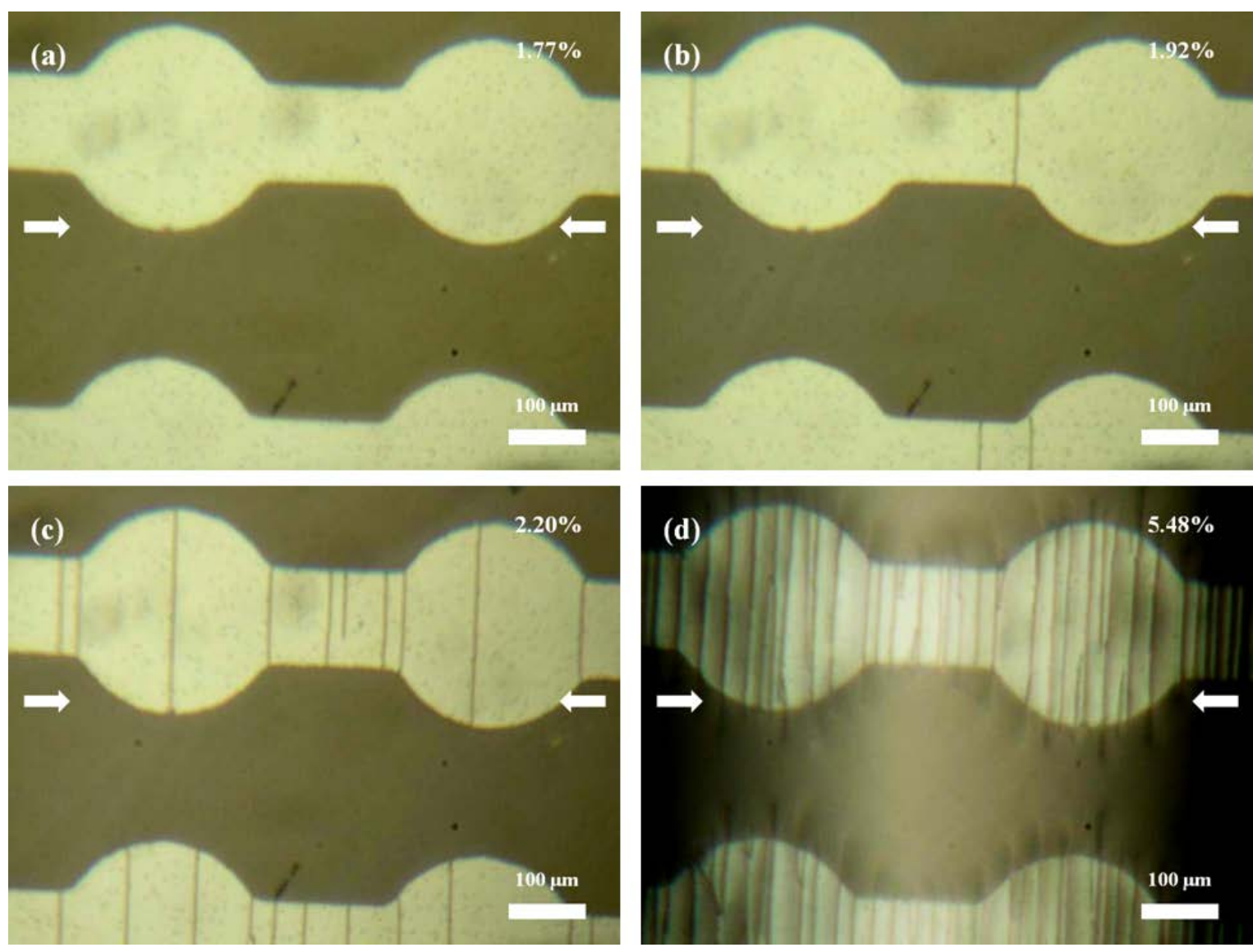

Fig. 6.6 Optical microscopy images of circular-patterned ITO coated PET films under compressive buckling deformation mode showing crack initiation and propagation up to $6 \%$ strain. Arrows indicate the buckling direction. 

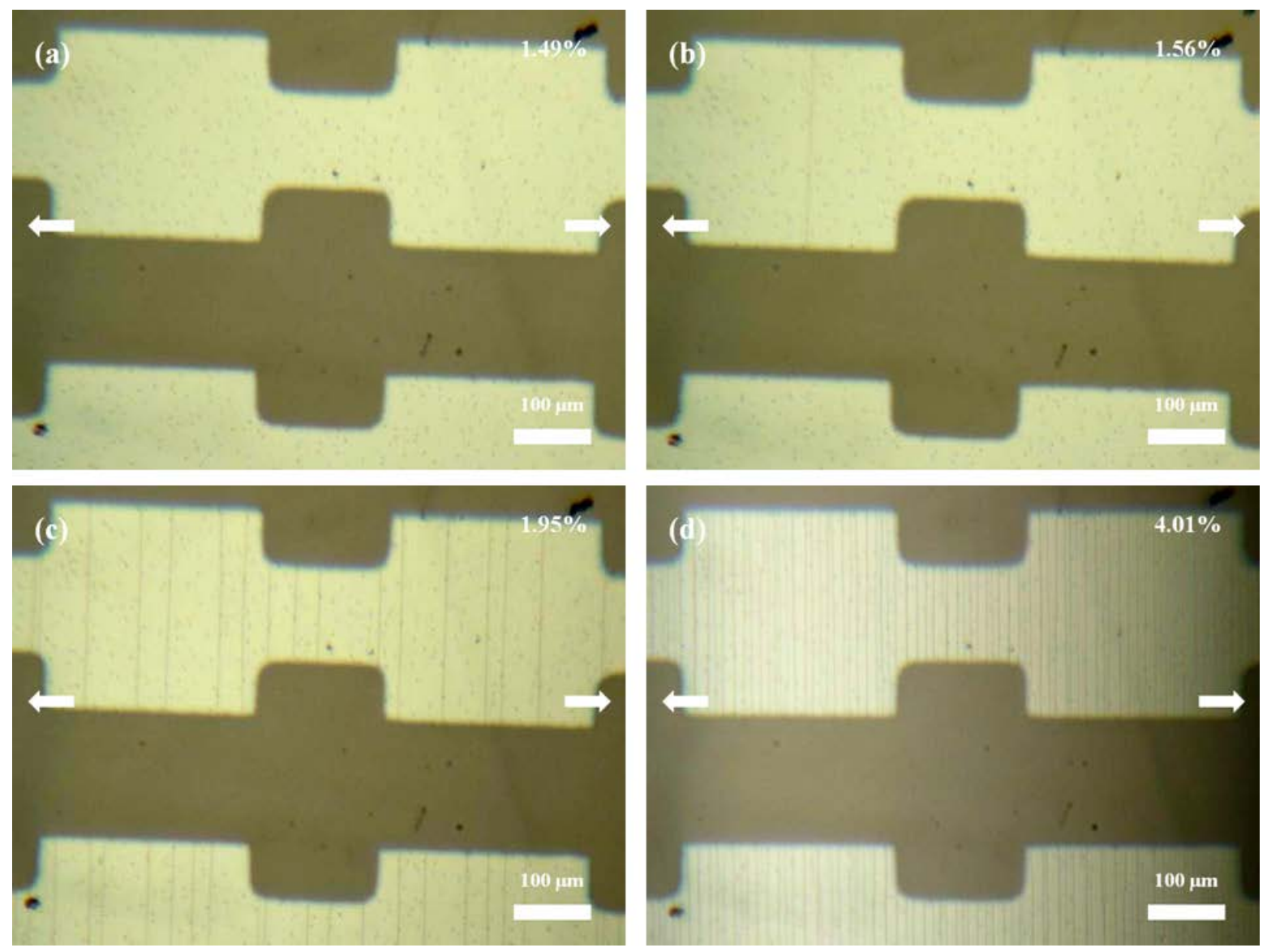

Fig. 6.7 Optical microscopy images of square-patterned ITO coated PET films under tensile buckling deformation mode showing crack initiation and propagation up to 6\% strain. Arrows indicate the buckling direction. 

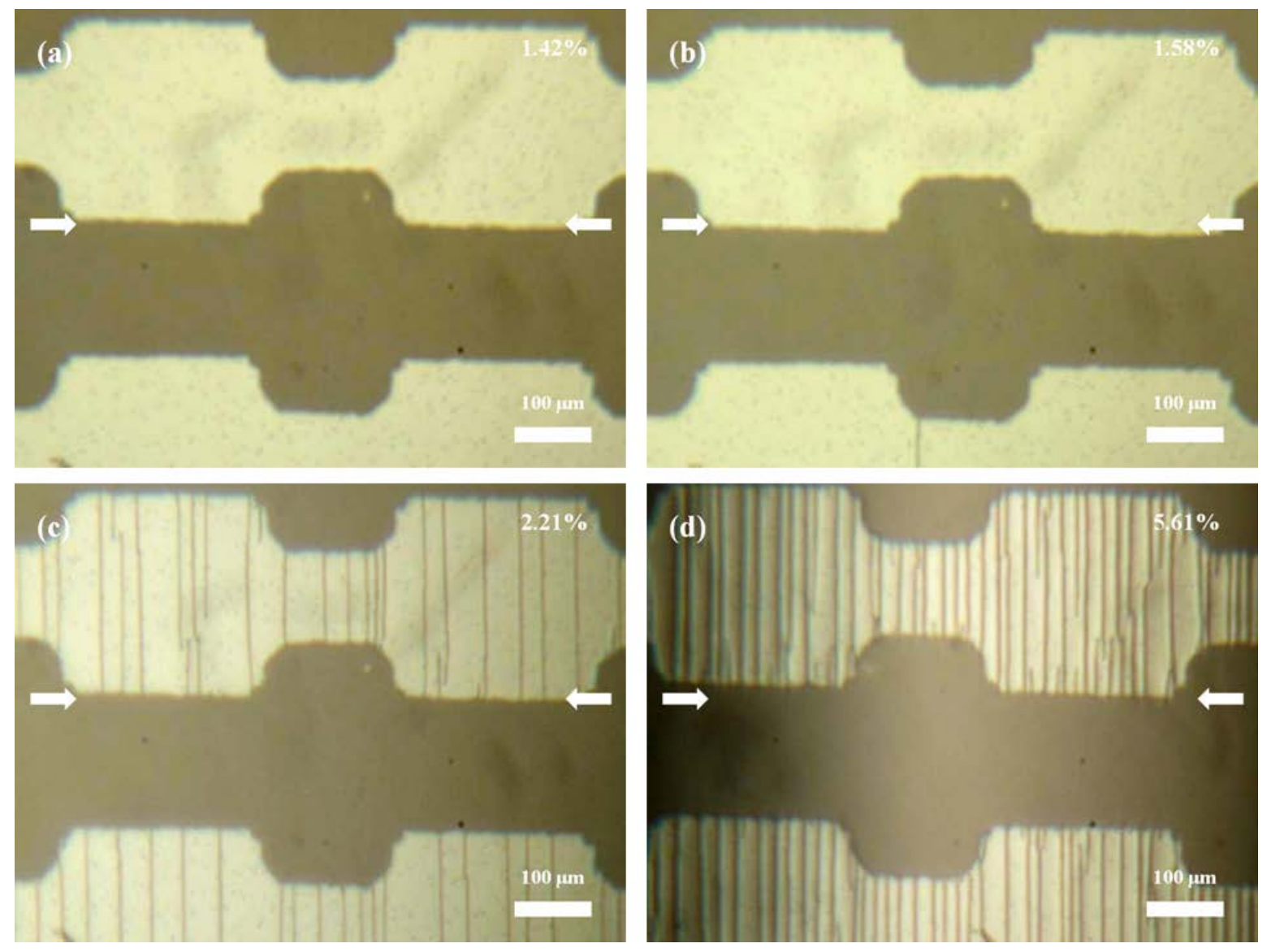

Fig. 6.8 Optical microscopy images of square-patterned ITO coated PET films under compressive buckling deformation mode showing crack initiation and propagation up to 6\% strain. Arrows indicate the buckling direction. 

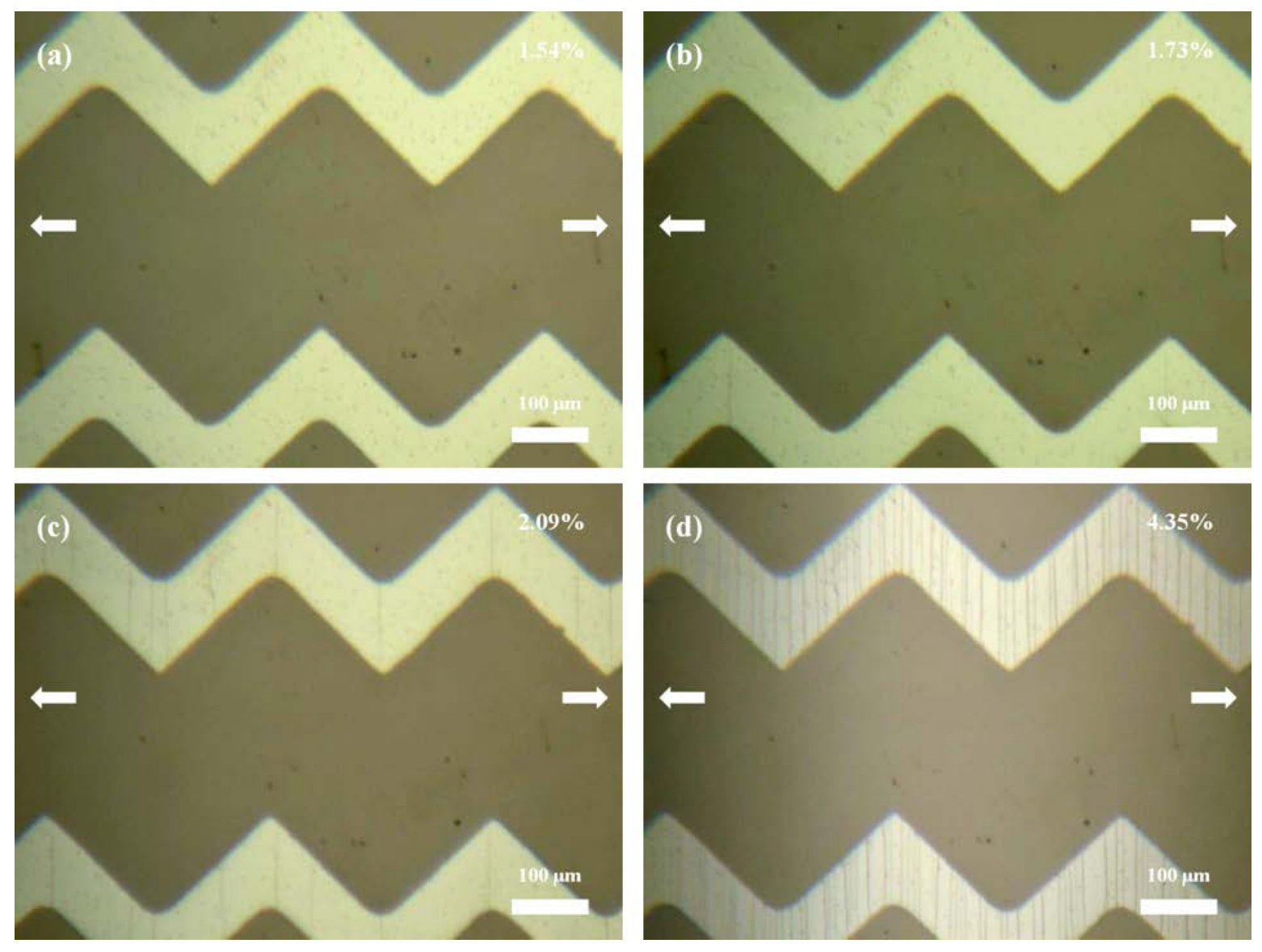

Fig. 6.9 Optical microscopy images of zigzag-patterned ITO coated PET films under tensile buckling deformation mode showing crack initiation and propagation up to 6\% strain. Arrows indicate the buckling direction. 

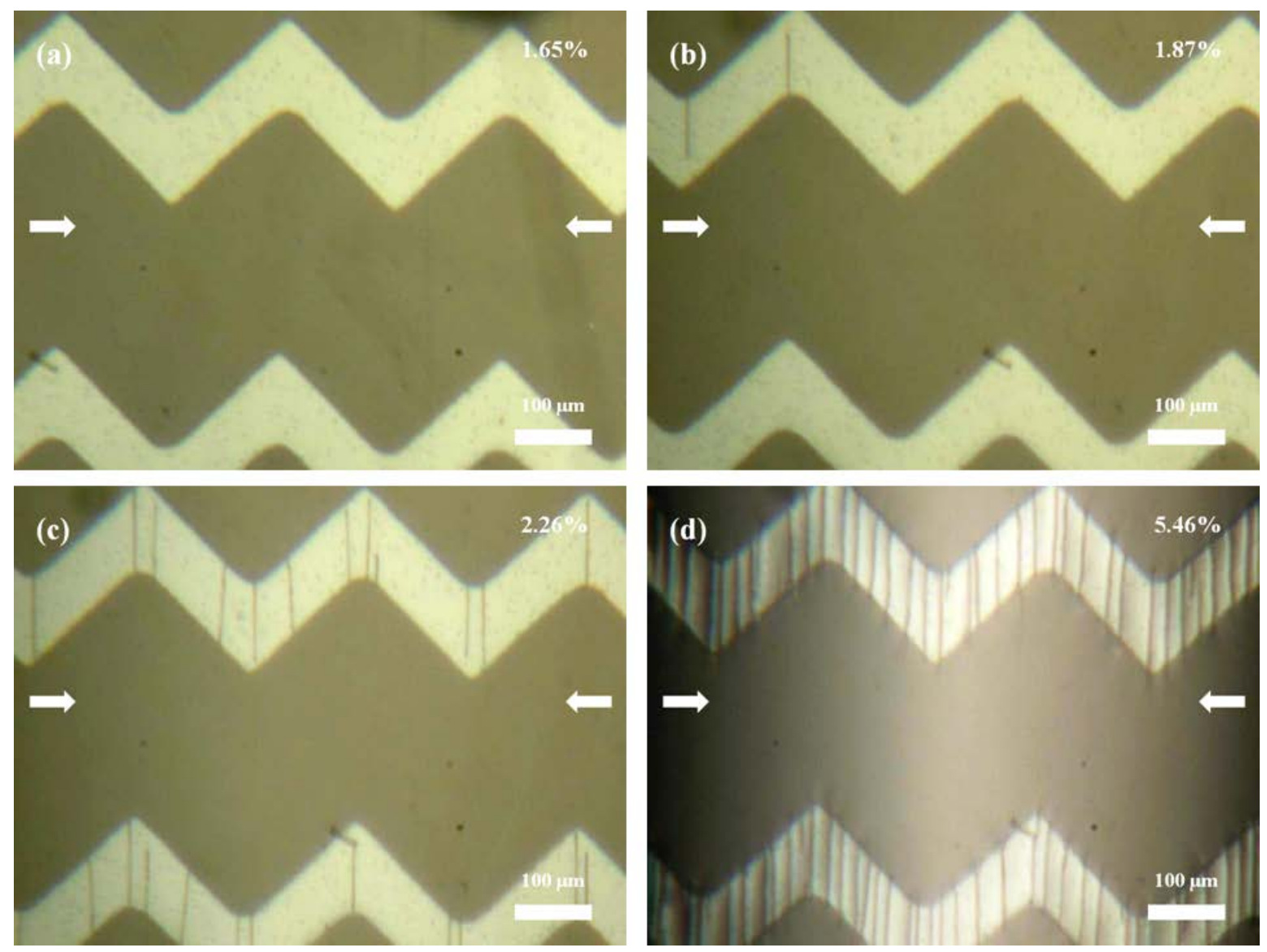

Fig. 6.10 Optical microscopy images of zigzag-patterned ITO coated PET films under compressive buckling deformation mode showing crack initiation and propagation up to 6\% strain. Arrows indicate the buckling direction.

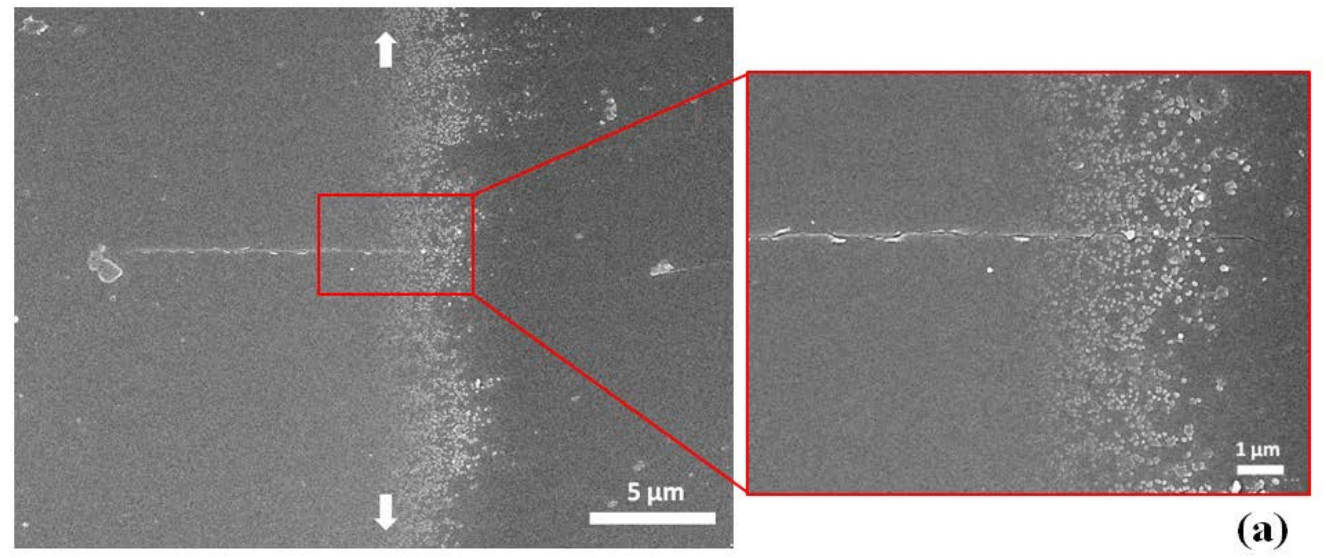




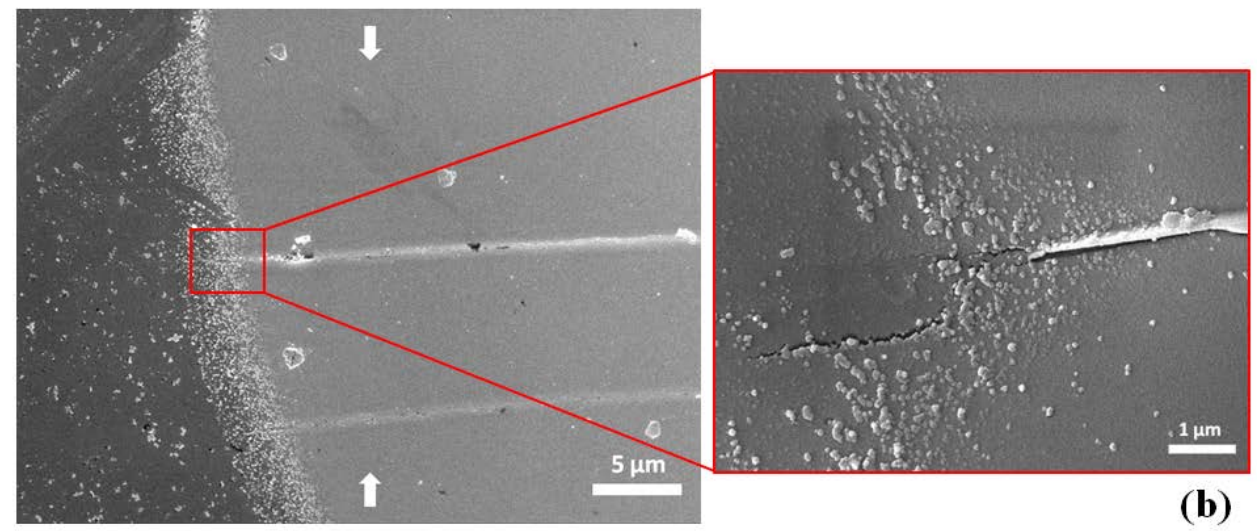

Fig. 6.11 SEM micrographs of patterned ITO coated PET films under (a) tensile and (b) compressive buckling deformation. Arrows indicate the buckling direction.

Fig. 6.12 shows COS-M values, for different pattern shapes and sizes, determined using the optical microscopy images. These values are found to be significantly higher than their COSR counterparts. The latter may suggests earlier failure initiation before cracks can be detected with optical microscopy. This is confirmed by the change in electrical resistance values (Fig. 6.13) at COS-M in which all specimens are observed to have greater than the $10 \%$ increase in resistance. These results are summarized in Table 6.1. It is believed that the role of the etch definition and initiation of edge micro-cracks may play a significant role. In particular, it is observed that the patterns under tension exhibit the largest deviation values between COS-M and COS-R. This can be related to the cohesive failure mode that is initiated at the pattern edges due to pre-mature cracking that is not detectable by optical microscopy. On the other hand smaller deviations are observed when the patterned film is under compression. This can be due to the lesser effect of the edge cracks on the patterned coating's delamination from the underlying substrate. 


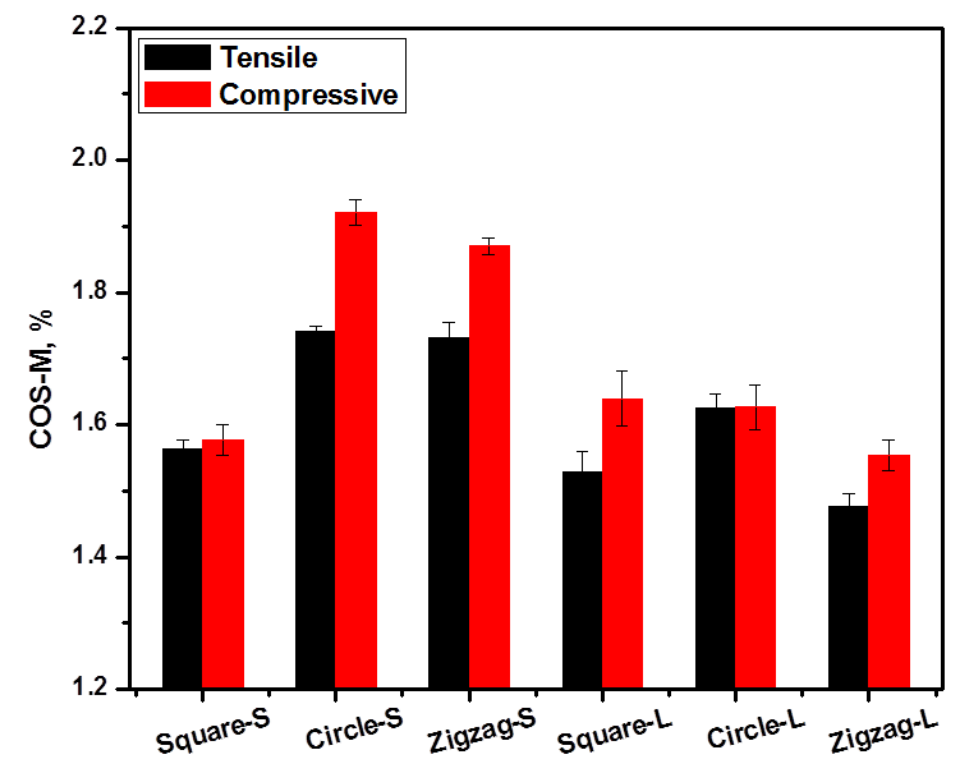

Fig. 6.12 Crack onset strain from in situ optical microscopy monitoring of ITO coated PET films.

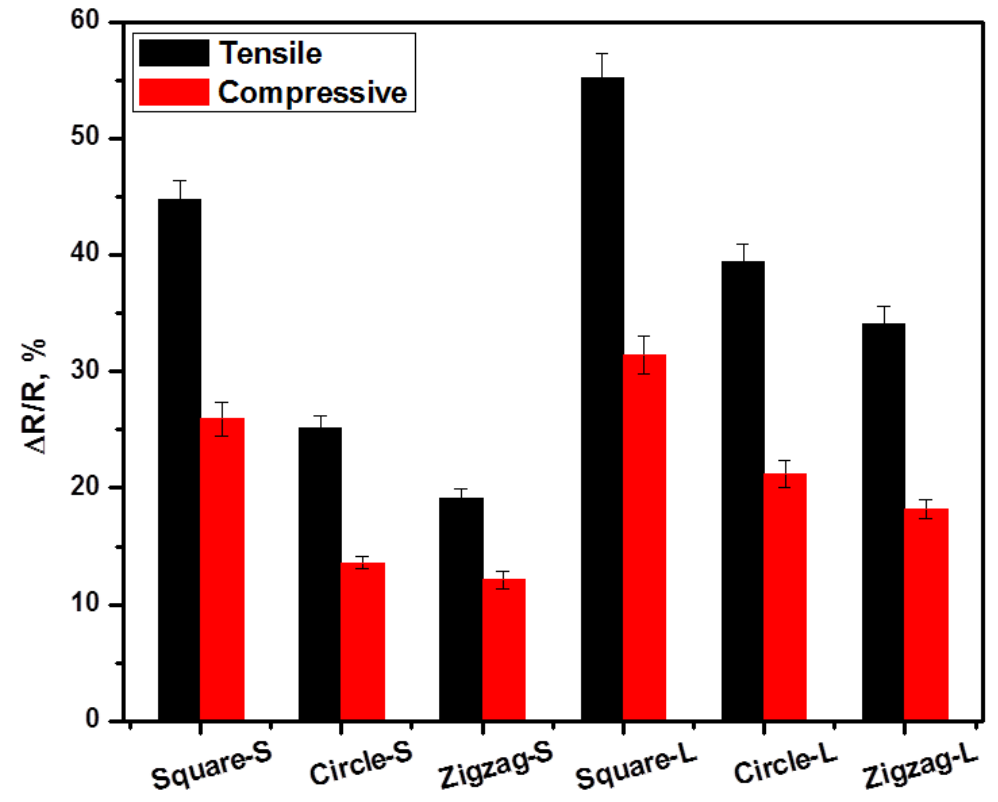

Fig. 6.13 Normalized electrical resistance at COS-M for ITO coated PEN films. 


\subsubsection{Design of experiments}

A further investigation of the effects of size and shape on the electrical degradation of patterned ITO films was conducted using DOE methods. Table 6.2 and 6.3 show the analysis of variance (ANOVA) for the main effects and their interaction for $\Delta \mathrm{R} / \mathrm{R}$ at COS-M under both tensile and compressive buckling conditions, respectively.

Table 6.2 Analysis of variance (ANOVA) for $\Delta \mathrm{R} / \mathrm{R}$ at COS-M under tensile buckling.

\begin{tabular}{lrrrrrr}
\hline Source & DF & Seq SS & Adj SS & Adj MS & F & P \\
\hline Size & 1 & 524.49 & 524.49 & 524.49 & 58.21 & 0.0000 \\
Shape & 2 & 1185.1 & 1185.1 & 592.55 & 65.76 & 0.0000 \\
Size*Shape & 2 & 11.59 & 11.59 & 5.79 & 0.64 & 0.5580 \\
Error & 6 & 54.06 & 54.06 & 9.01 & & \\
Total & 11 & 1775.24 & & & & \\
S= 3.00176 & R-sq $=\mathbf{9 6 . 9 5 \%}$ & R-sq (adj) $=\mathbf{9 4 . 4 2} \%$ & & & \\
\hline
\end{tabular}

Table 6.3 Analysis of variance (ANOVA) for $\triangle \mathrm{R} / \mathrm{R}$ at COS-M under compressive buckling.

\begin{tabular}{lrrrrrr}
\hline Source & DF & Seq SS & Adj SS & Adj MS & F & P \\
\hline Size & 1 & 121.78 & 121.78 & 121.78 & 23.57 & 0.0030 \\
Shape & 2 & 420.51 & 420.51 & 210.26 & 40.69 & 0.0000 \\
Size*Shape & 2 & 2.57 & 2.57 & 1.29 & 0.25 & 0.7870 \\
Error & 6 & 31 & 31 & 5.17 & & \\
Total & 11 & 575.87 & & & & \\
$\mathbf{S = \mathbf { 2 . 2 7 3 0 6 }}$ & R-sq $=\mathbf{9 4 . 6 2 \%}$ & R-sq (adj) $=\mathbf{9 0 . 1 3 \%}$ & & & \\
\hline
\end{tabular}

In both cases, the design factors are found to be significant since their P-values are less than the confidence level $(\alpha=0.05)$. However, their interaction is observed to be insignificant. From the main effects plots (Fig. 14), the shape of the patterns is more significant than their size. 
This may be due to the geometrical variables - change in area $\left(A_{1} / A_{2}\right)$, sharpness, and number of corners - present in the patterns are associated with the shape factor. Also, square-shaped patterns observed to exhibit a higher change in electrical resistance values. This is due to the fact that they have the highest combinations of geometrical variables.
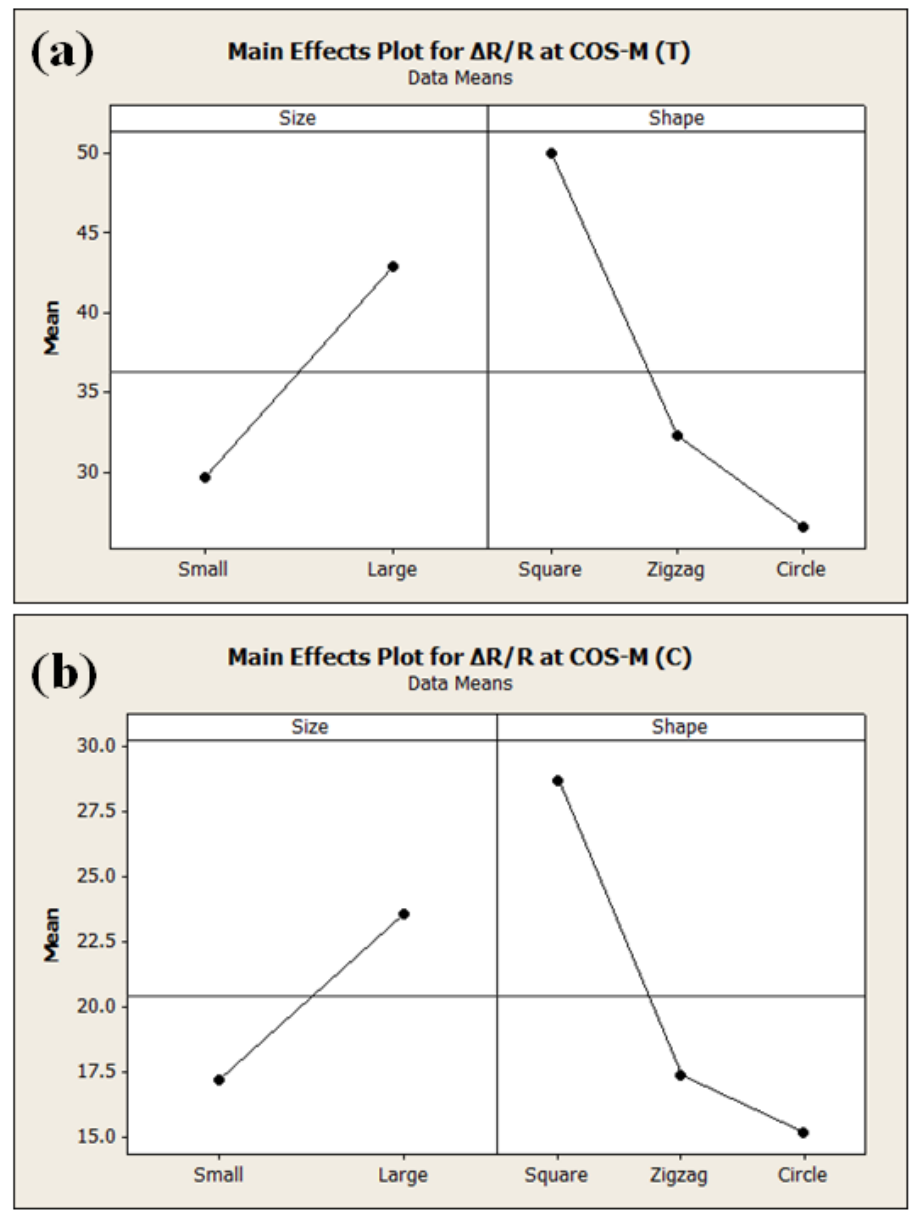

Fig. 6.14 Main effects plot for change in electrical resistance at COS-M for ITO films under: (a) tensile and (b) compressive buckling.

The significant contribution of size on the $\Delta \mathrm{R} / \mathrm{R}$ at COS-M is shown by the interactions plots (Fig. 15). This is in agreement with previous work by Bouten et al. [69] who investigated 
the mechanical behavior of ITO line patterns. Using the two-point bending method on narrow patterned ITO lines on Arylite substrates, they obtained COS values of 1.35, 1.29, 1.22, and $1.19 \%$, for ITO lines of 10, 30, 100, and $300 \mu \mathrm{m}$ width, respectively.

The reliability of the ANOVA results is confirmed by the normal probability plots of the randomly distributed residuals around the mean as shown in Fig. 16.
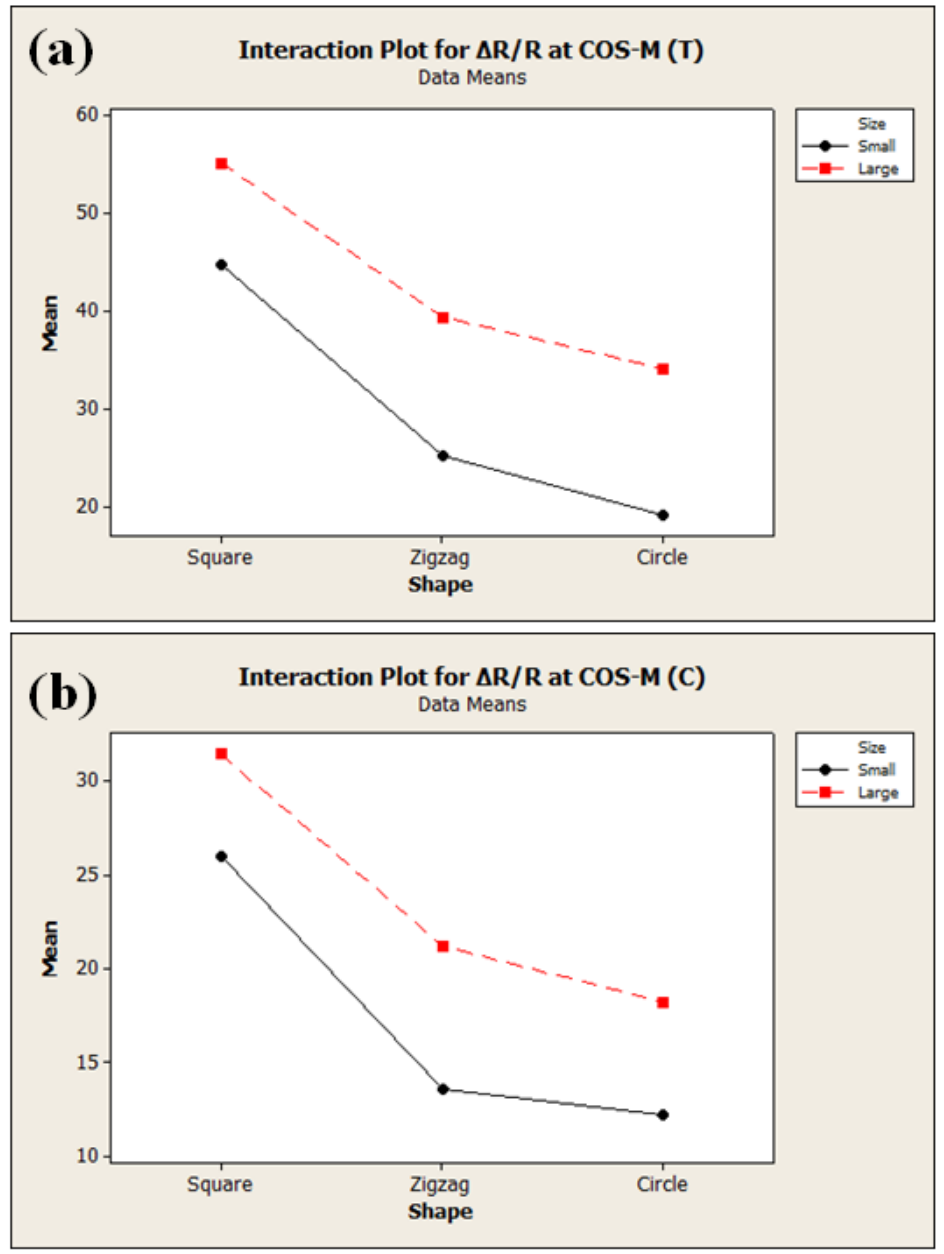

Fig. 6.15 Interaction plot for change in electrical resistance at COS-M for ITO films under: (a) tensile and (b) compressive buckling. 

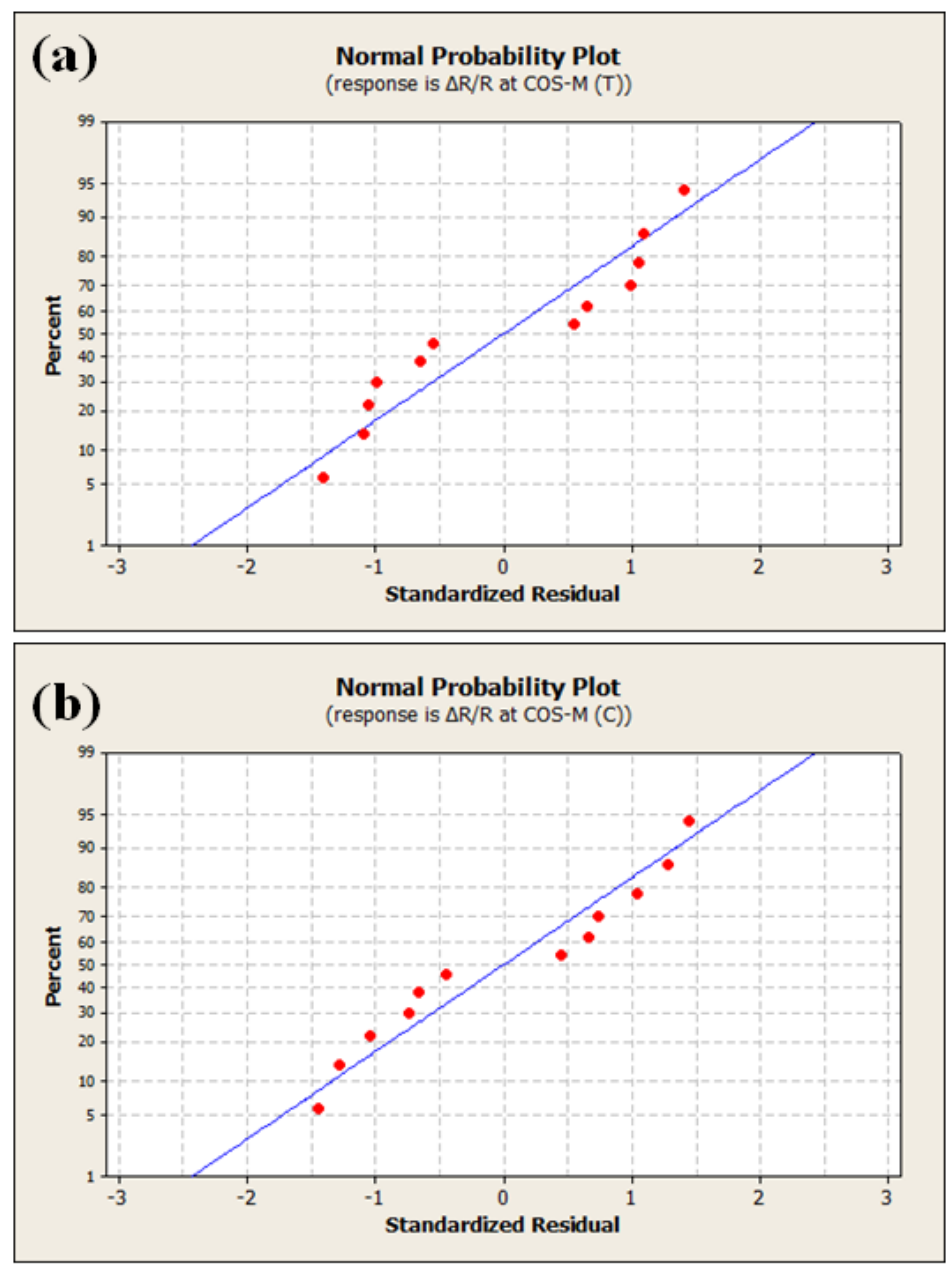

Fig. 6.16 Normal probability plot for change in electrical resistance at COS-M for ITO films under: (a) tensile and (b) compressive buckling. 


\section{TRIBO-CORROSION OF FLEXIBLE TRANSPARENT ELECTRODES}

\subsection{Introduction}

The efficiency and long term reliability of multilayered films of Ag/Ag-alloy based ITO used in solar energy applications depend on the surface integrity of the film. Surface integrity can be breached due to exposure to a harsh outdoor environment and mishandling. It is therefore important to study the tribo-corrosion properties of such film systems.

Surface integrity and durability of solar materials can be an important issue in solar energy applications such as heat mirrors used in flat-plate collectors, solar heating, concentrating solar power systems, low-emissivity windows and spectrally selective surfaces. This is because damaged surfaces can lead to a degradation of optical components and thus to a compromised system efficiency. Therefore, the widespread adoption of such technologies depends on the development of highly durable and low cost solar films deposited on rigid and flexible substrates [131-133].

In this chapter a combined tribological and corrosion approach using ITO/Ag/ITO and ITO/Ag-alloy/ITO films in aggressive salt environments is undertaken in order to investigate the suitability of such model systems as durable components in solar energy and energy-efficient applications that are currently emerging. Investigation of the tribo-corrosion behavior of the electrodes is critical in the design and manufacturing of reliable flexible devices which will remain functional after repetitive mechanical flexing under harsh environmental conditions. Also, understanding the tribo-corrosion properties of multilayers can lead to increasing efficiency and long term reliability of such electrode components. 


\subsection{Results and discussion}

\subsubsection{In-situ electrical resistance measurements}

In-situ electrical resistance measurements can be an invaluable tool for assessing the reliability of conductive multilayers. This is because electrical resistance changes during testing can be associated with surface changes due to wear and corrosion processes [88].

Fig. 7.1 shows the electrical resistance change with number of reciprocating cycles with focus on the initial stage of the experiment and up to 540 reciprocating cycles.

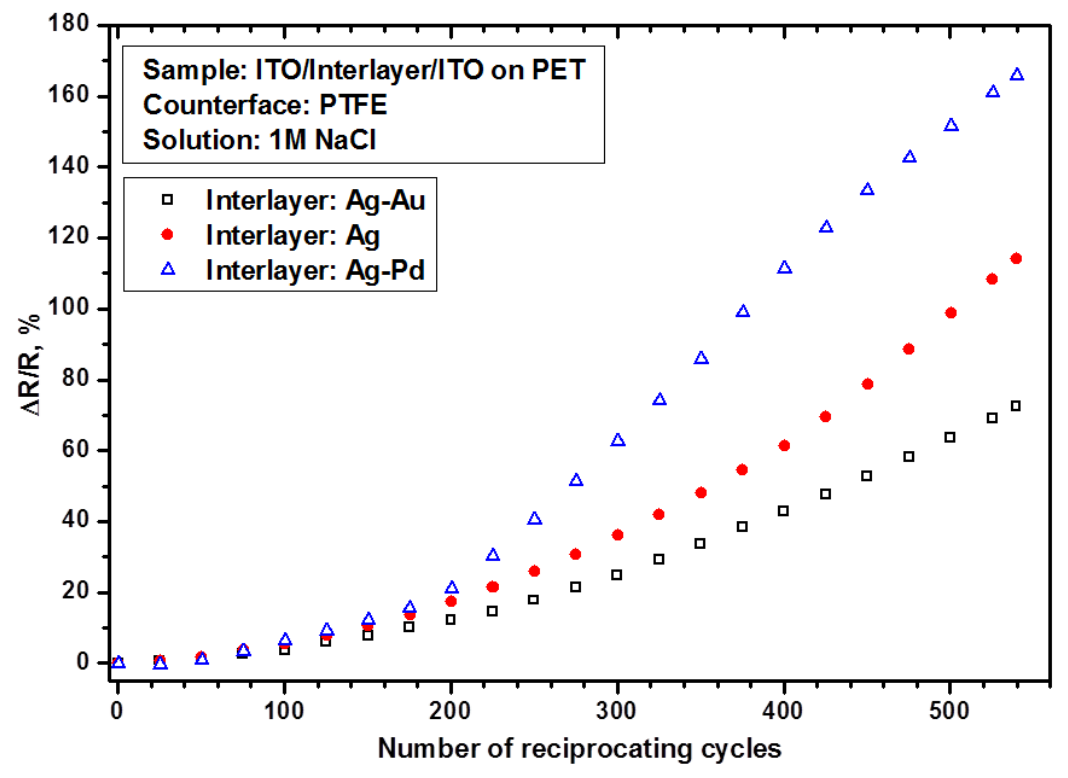

Fig. 7.1 In-situ electrical resistance measurement against number of reciprocating cycles. 
The smoothest electrical resistance change is observed in the case of the ITO/Ag-Au/ITO multilayer underlying the corrosion resistance to $\mathrm{NaCl}$ of $\mathrm{Au}$ and its importance as an alloying compound. On the other hand, after approximately 200 reciprocating cycles a sharp increase in resistance change is observed for the case of the ITO/Ag-Pd/ITO sample. This is a surprising result, regarding the contribution of corrosion to the process, since the ITO/Ag/ITO sample could exhibit the sharpest electrical resistance increase since it is not alloyed. This may indicate reduced adhesion between the ITO and the Ag-Pd layer.

A change of $20 \%$ in electrical resistance can be defined as a typical coating failure initiation point in this case. Fig. 7.2 shows a bar graph for the three samples under investigation and their 20\% resistance change values during reciprocating sliding against a PTFE ball in $\mathrm{NaCl}$ $1 \mathrm{M}$ solution. It is observed that failure initiation for the ITO/Ag-Pd/ITO sample occurs at around 200 reciprocating cycles and then the ITO/Ag/ITO sample follows with a failure initiation point at around 219 cycles. The Ag-Au based sample exhibits the most durability with failure initiation corresponding to 268 reciprocating cycles.

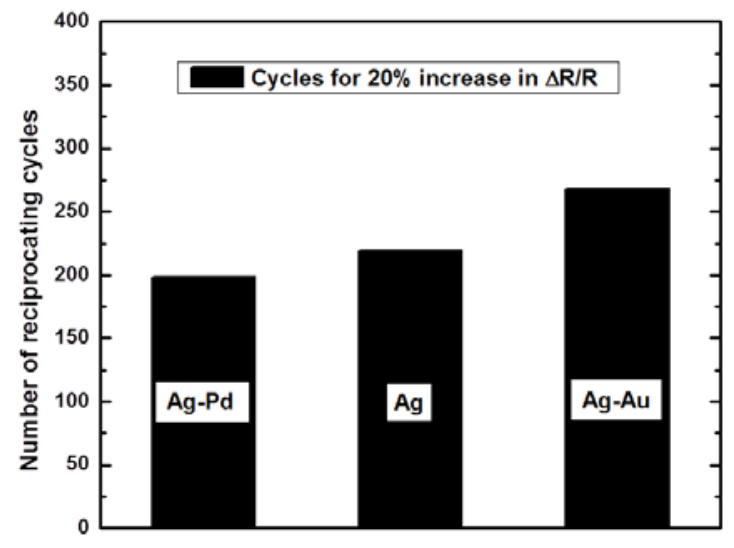

Fig. 7.2 Critical number of reciprocating cycles for 20\% electrical resistance increase. 


\subsubsection{Weight measurements}

Weight measurements before and after wear testing were conducted in order to determine the wear behavior of sliding surfaces. In this work both the multilayer surface and the PTFE ball counterbody were weighed before and after testing.

Fig. 7.3 shows the relation of weight loss with the number of reciprocating cycles in $1 \mathrm{M}$ $\mathrm{NaCl}$ solution. Minimal weight change is observed up to 540 reciprocating cycles for all three samples. Above the 540 cycle threshold, a rapid wear regime is observed until around 1080 reciprocating cycles. Above 1080 cycles a plateau regime is observed mostly for the ITO/Ag/ITO and the ITO/Ag-Au/ITO samples. The ITO/Ag-Pd/ITO multilayer is observed to a weight loss above 1080 reciprocating cycles.

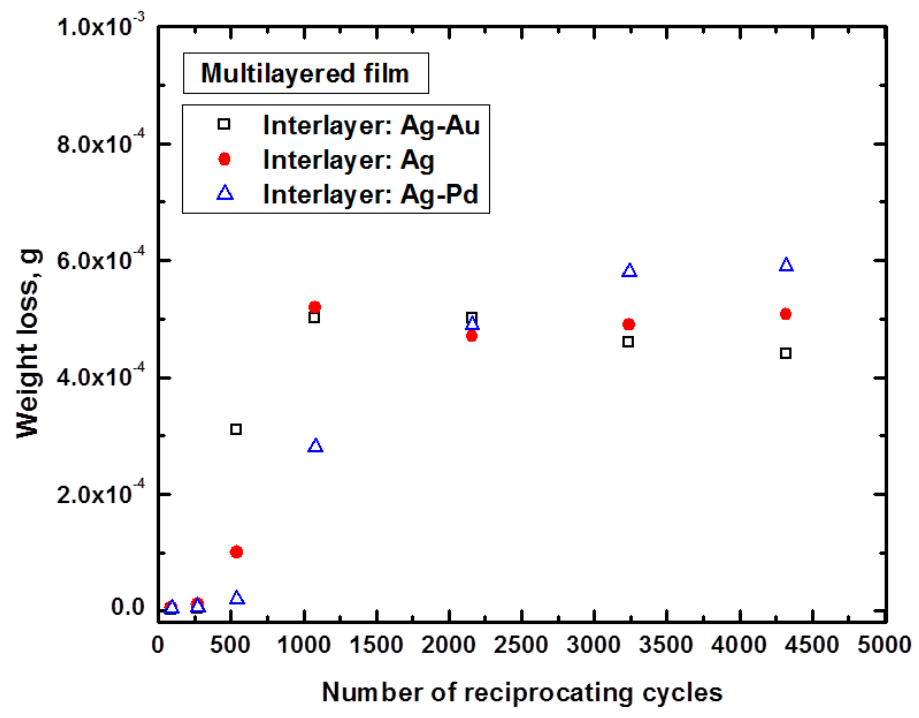

Fig. 7.3 Graph showing the weight loss of multilayer films versus number of reciprocating cycles in $1 \mathrm{M} \mathrm{NaCl}$ solution. 
However, it is essential to measure the weight loss of the counterbody (PTFE) ball and consider it with respect to the multilayer wear. This is important because the PTFE surface is wearing with increasing reciprocating cycles, as shown in Fig. 7.4, resulting in a counterformalto-conformal contact transition.

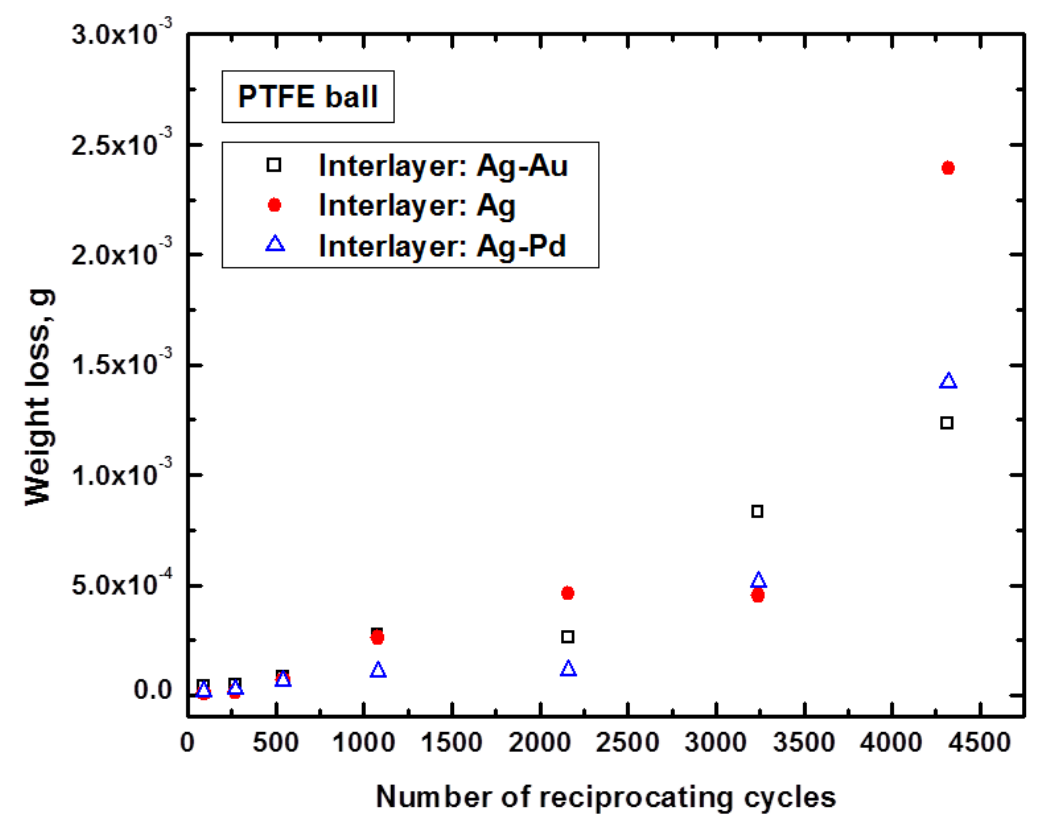

Fig. 7.4 Graph showing the weight loss of the PTFE sliding balls with respect to number of reciprocating cycles in $1 \mathrm{M} \mathrm{NaCl}$ solution.

Minimal weight loss is observed for tests below 540 reciprocating cycles suggesting minimal wear of the ball surface. This can also be attributed to the low friction coefficient of the PTFE material. More significant PTFE wear is observed above 540 sliding reciprocating cycles in $1 \mathrm{M} \mathrm{NaCl}$. This is mostly observed for balls sliding against the Ag and Ag-Au alloy-based ITO surfaces. At a high number of reciprocating cycles (above 3240) a rapid PTFE weight loss, for all 
samples, is observed suggesting possible material transfer and impregnation on to the worn polymer substrate.

\subsubsection{X-ray photoelectron spectroscopy}

Multilayer worn surface investigation was conducted using XPS in order to assess the combined effect of wear and corrosion. XPS surface scans were conducted for all three samples and for the whole range of reciprocating cycles including untested samples for comparison purposes. Typical x-ray photoelectron spectra are shown in Fig. 7.5.

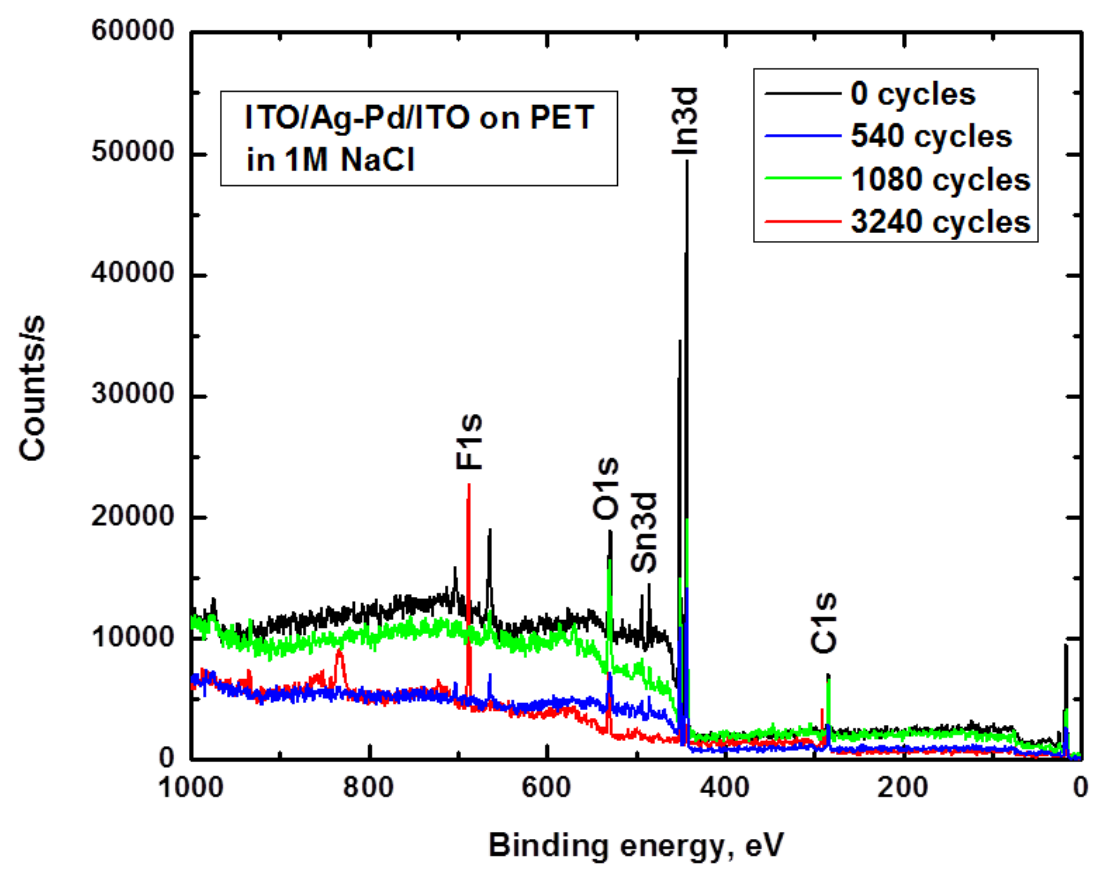

Fig. 7.5 Typical x-ray photoelectron spectra for the case of Ag-Pd based ITO multilayer and increasing number of reciprocating cycles. 
XPS confirms the transfer and impregnation of PTFE material on to the worn polymer surface at high reciprocating cycles. A fluorine peak at $688 \mathrm{eV}$ is observed to first occur at 3240 cycles. It can then be suggested that some of the PTFE debris particles, previously acted as abrasion particles, are impregnating the soft polymer substrate. This observation is consistent for all sample surfaces. Another important information that can be extracted from observing the XPS graph is the diminishing intensity of both the In and Sn peaks with increasing number of reciprocating cycles indicating progressive wear of the ITO layers until total exposure of the underlying substrate. Fig. 7.6 shows a graph of the atomic percentage in In concentration versus the number of reciprocating cycles as measured by XPS.

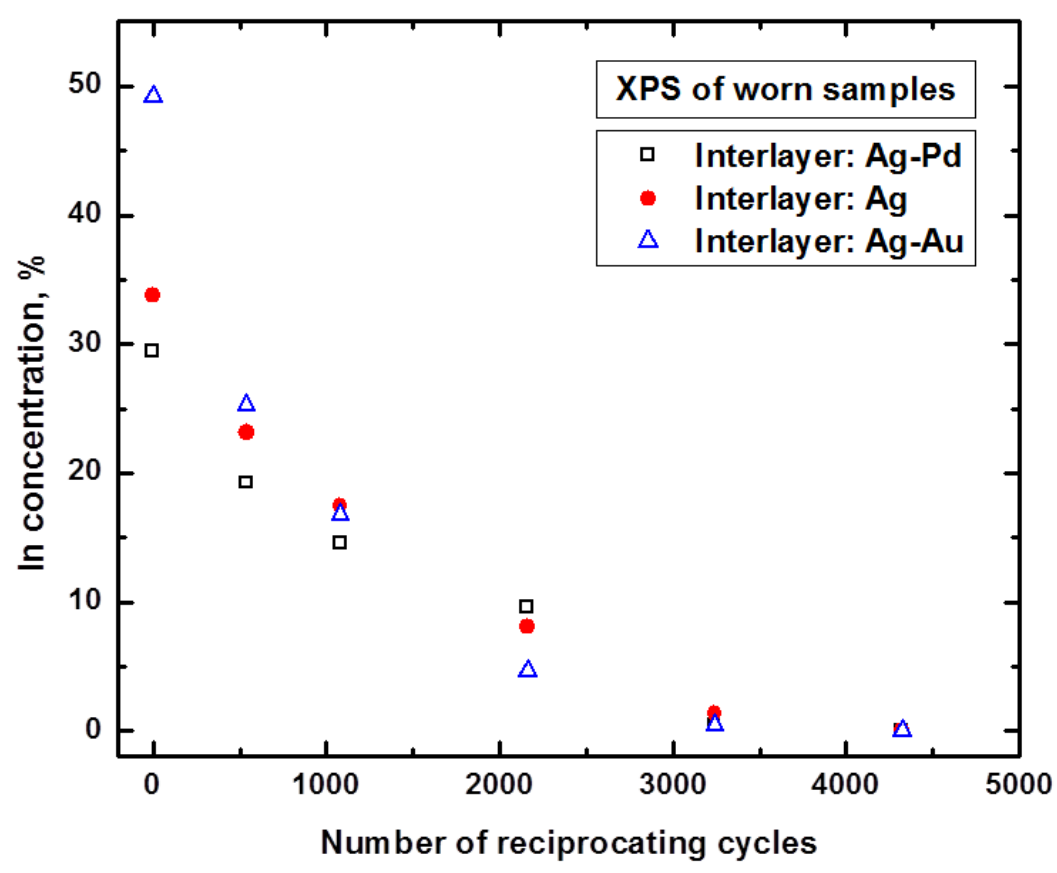

Fig. 7.6 Graph showing In atomic percentage concentration against number of reciprocating slides for all three samples sliding against PTFE balls in $1 \mathrm{M} \mathrm{NaCl}$ solution. 
It is observed that the ITO layers are almost totally removed at 3240 cycles for all samples since the In concentration has decayed approaching $0 \%$. This coincides with the PTFE material transfer mechanism to the polymer substrate. There is no detectable In concentration above 3240 reciprocating cycles suggesting total ITO removal.

\subsubsection{Electrochemical impedance spectroscopy}

EIS is used in order to assess the effect of wear on the corrosion resistance of the Ag and Ag-alloy-based ITO structures during the initial stages of the tribo-corrosion process and up to 540 reciprocating cycles. Fig. 7.7 shows a representative Nyquist plot for Ag-Au -based ITO samples worn up to 540 cycles. The complex impedance at each frequency is usually expressed by its real component (Z') and its imaginary component (Z") in a Nyquist plot. The Nyquist plot (Fig. 7.7) shows two time constants; one at higher frequencies as shown in the inset indicating ITO top layer breakdown and another at lower frequencies suggesting corrosion of the interlayer. It is observed that as the number of reciprocating cycles increases, the corrosion resistance of the structure is decreasing. This is highlighting the significant role that the tribology component is playing in the corrosion process.

The equivalent circuit diagram shown in the inset in Fig. 7.8 consists of a solution resistance $R_{s}$, a film resistance due to defects $R_{f}$, a film capacitance $C_{f}$, a constant phase element CPE and a charge transfer resistance $\mathrm{R}_{\mathrm{ct}}$. This is a common model used to investigate the localized corrosion of coatings exhibiting pinholes and other permeable defects. When in contact with the coating the electrolyte solution is penetrating it at defect sites and corrosion reactions are occurring at the interface of the top coating and the interlayer [122]. Fig. 7.8 reveals a 
decreasing trend of charge transfer resistance $\mathrm{R}_{\mathrm{ct}}$ with increasing number of reciprocating cycles thus indicating an increasing corrosion rate with increasing number of cycles because of removal of the top layer due to the sliding wear process. The decrease is more pronounced in the ITO/Ag/ITO case suggesting the importance of Ag-alloying for corrosion protection against harsh salt-containing environments. It has been reported [122] that corrosion reactions of Ag exposed to $\mathrm{NaCl}$ include formation of $\mathrm{AgCl}$ which is an anodic polarization of the sample working electrode.

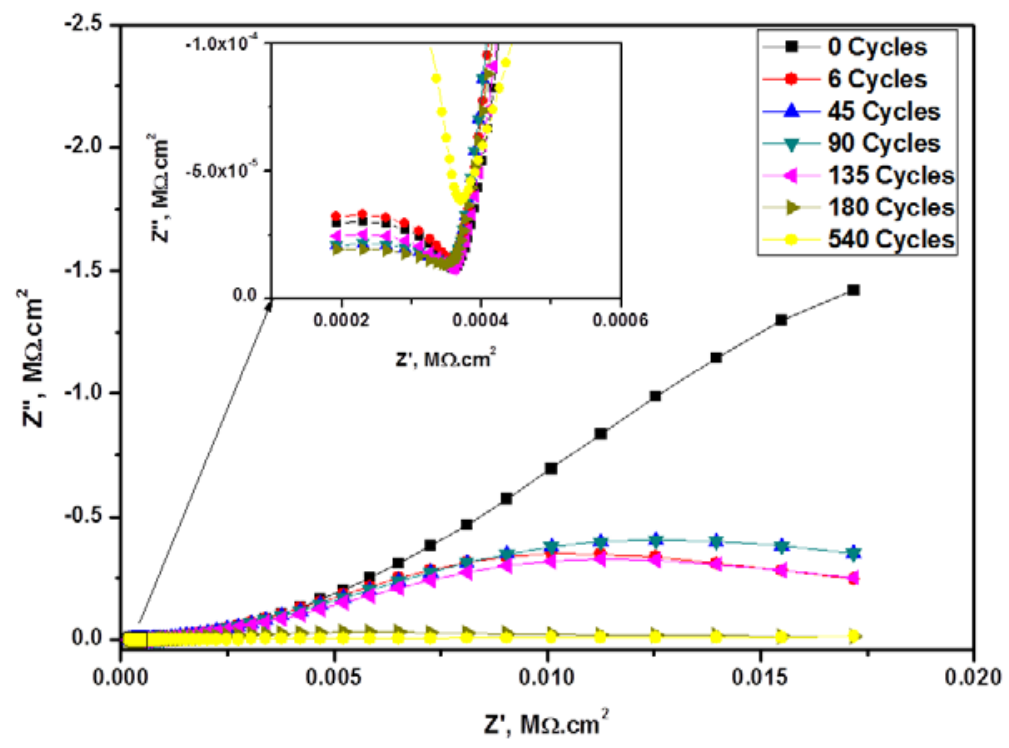

Fig. 7.7 Typical Nyquist plot for ITO/Ag-Au/ITO structures worn for up to 540 reciprocating cycles. 


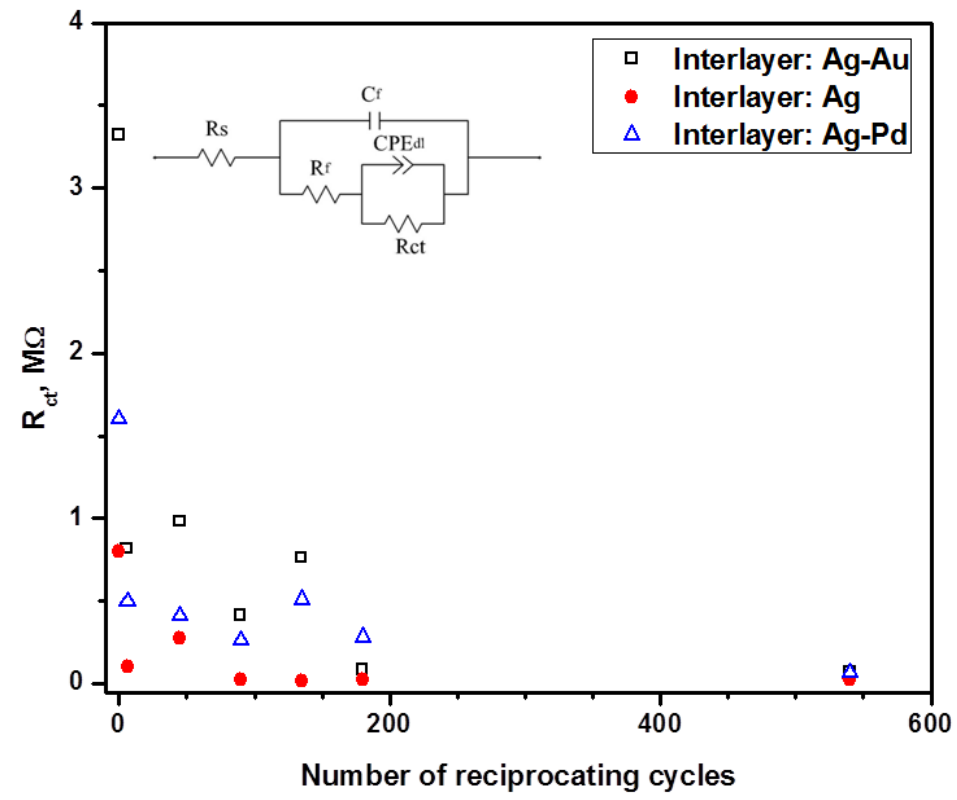

Fig. 7.8 Charge transfer resistance versus number of reciprocating cycles. Inset shows the equivalent circuit diagram used to model the multilayer structure.

\subsubsection{Microscopic characterization}

\subsubsection{PTFE counterbody}

In most cases cohesive wear in the form of abrasion by the ITO counter surface asperities and debris particles is observed for the PTFE counterbody. The PTFE surface and subsurface material deforms plastically. This is caused by counterface asperities and ITO debris fragments. The permanently deformed ridges are oriented parallel to the reciprocating sliding direction and their density increases with increasing number of reciprocating cycles. Some plastically deformed areas are also observed as shown in the two sides of Fig. 7.9a indicating ploughing and 
cutting of the polymer. In the middle of Fig. 7.9a a lump of debris is also observed. This is confirmed by Fig. 7.9b showing a 3D profile of the worn PTFE ball.
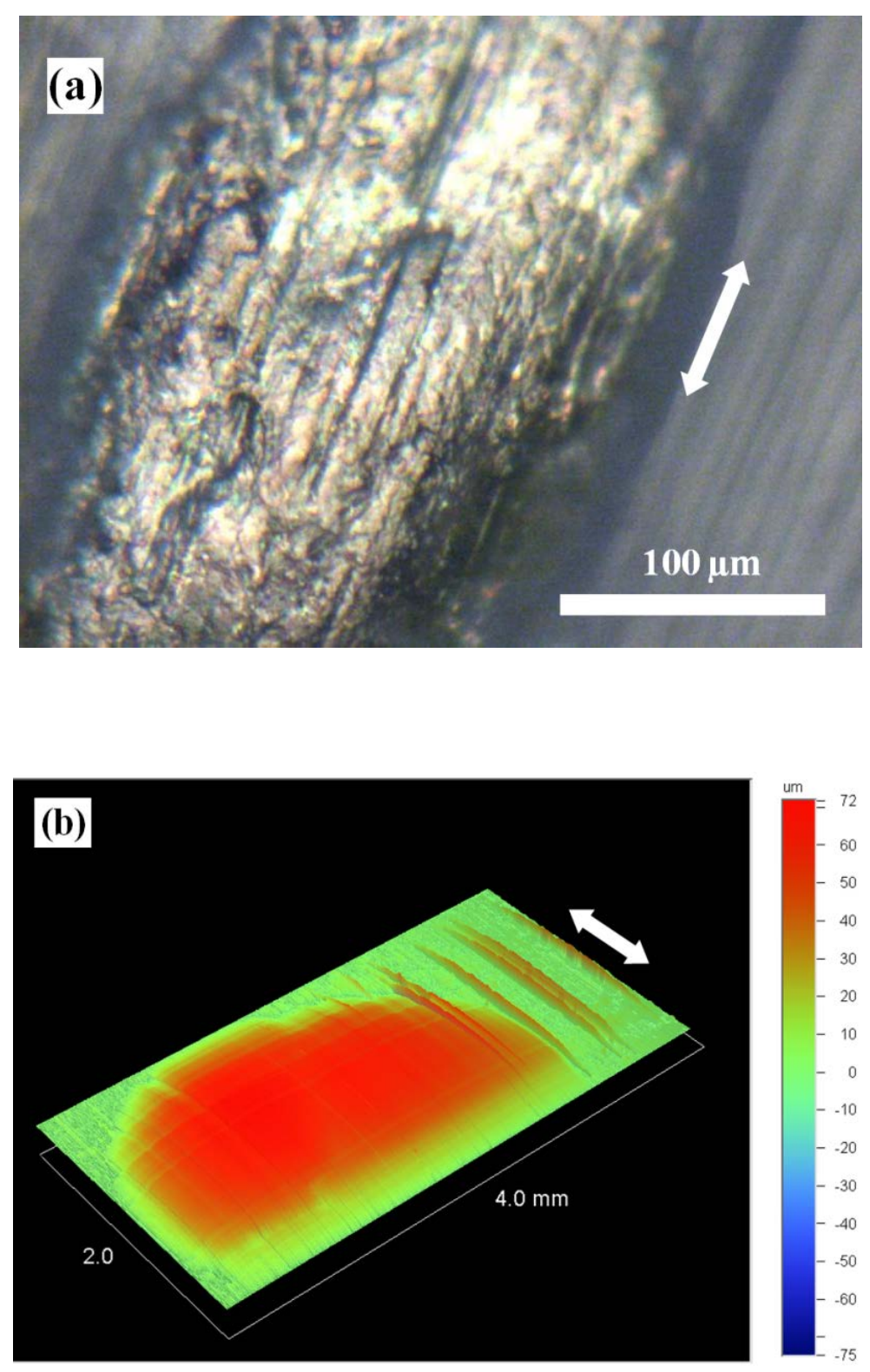

Fig. 7.9 (a) Optical microscopy image of a PTFE ball surface sliding against an ITO/Ag/ITO surface in reciprocating motion for 4320 cycles, (b) 3-D surface profile of the same PTFE surface. Arrows indicate reciprocating motion direction. 


\subsubsection{Multilayered sample surfaces}

At low numbers of reciprocating cycles and up to 540 cycles, microcutting is observed as shown in Fig. 7.10a for the case of an ITO/Ag-Au/ITO samples worn for 540 cycles. Large regions of delaminated coating are observed with some corrosion initiation within them. When the number of reciprocating cycles increases to 3240 cycles (Fig. 7.10b) the wear track becomes well defined and the underlying polymer substrate is exposed. The multilayered film is mostly removed by the combined action of the counterbody and the corrosive environment. Ploughing and plastic deformation of the underlying substrate is noted as well as PTFE material impregnation.

Microcutting accompanied with brittle ITO coating fragment failure and initiation of coating removal is also observed for a relatively low number of reciprocating cycles, below 540, as shown in Fig. 7.11. This is consistent with XPS data which shows a gradual decrease of the In concentration even at the low reciprocating cycle regime.

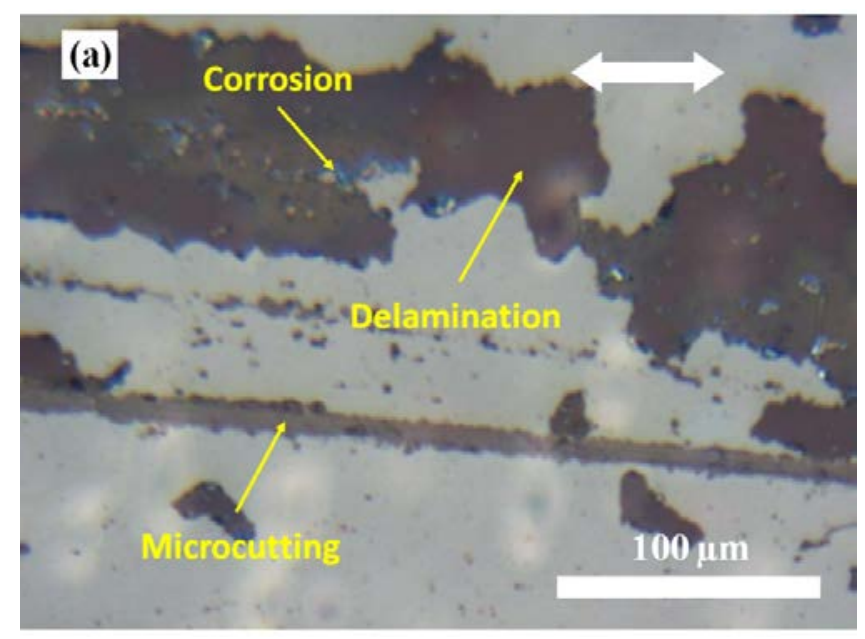




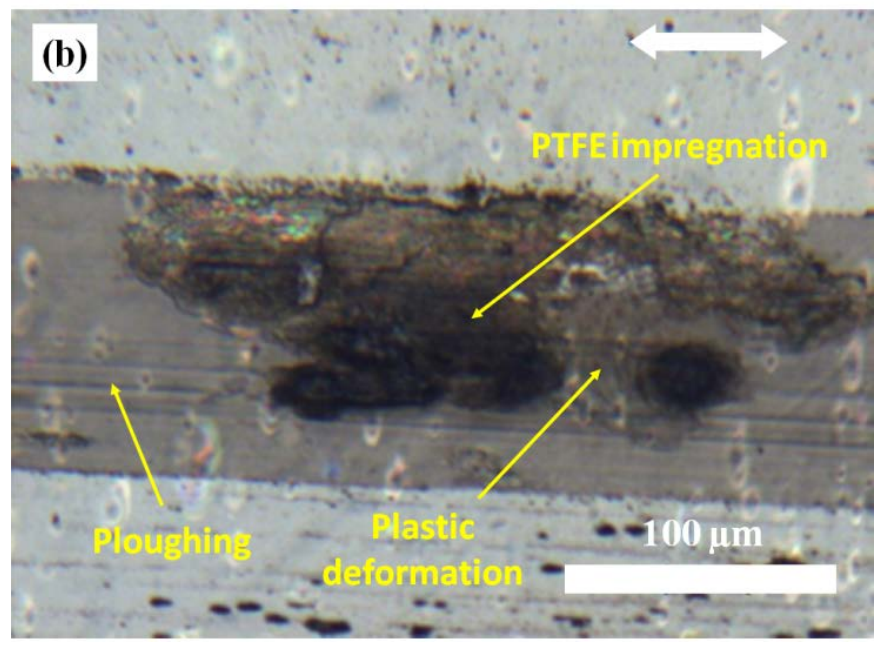

Fig. 7.10 (a) Optical image of a worn ITO/Ag-Au/ITO sample surface for 540 reciprocating cycles. Scale bar is the same for both images and equal to $0.1 \mathrm{~mm}$ (b) Optical image of a worn ITO/Ag-Au/ITO sample surface for 3420 reciprocating cycles. The double-headed arrow is the same for both images and indicates reciprocating direction.

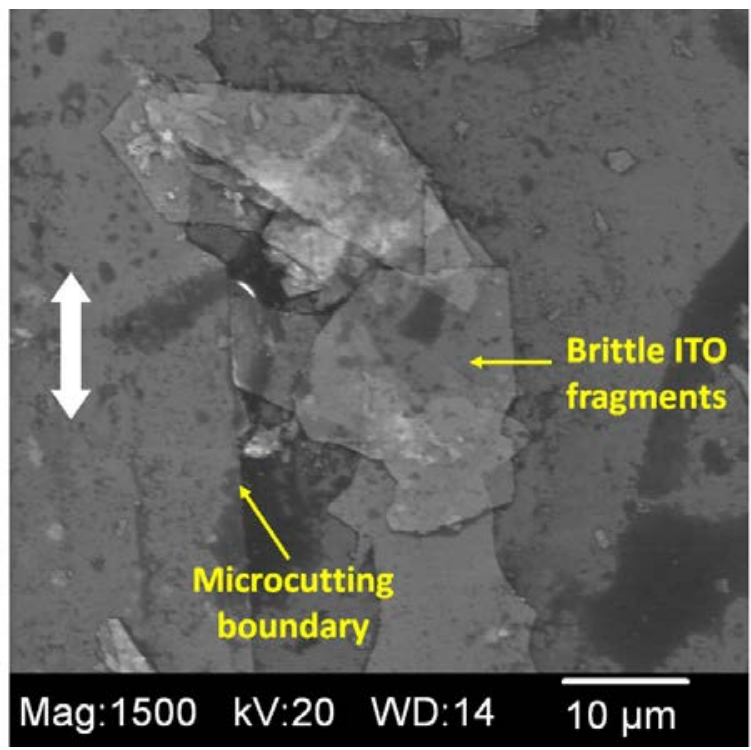

Fig. 7.11 SEM image of a worn ITO/Ag-Au/ITO sample surface for 270 reciprocating cycles. Double-headed arrow indicates the reciprocating direction. 


\section{CONCLUSIONS}

The motivation of this research was to investigate the electromechanical and mechanochemical degradation behavior of flexible transparent electrodes which are used as anodes in various optoelectronic device applications. The electrodes may corrode due to acidcontaining materials present in the device stacks. In addition, structural failure may occur due to external applied loading. It is therefore important to characterize the electrodes under different experimental methods which are all simulate induced stress and failure during manufacturing and in-service condition.

A substantial amount of effort was directed towards testing such electrodes under different stress states and deformation modes. This effort was divided into four parts: the development of new testing techniques, the actual testing of various electrodes, the finite element analysis, and the statistical analysis.

- A fatigue-corrosion apparatus was custom-built in order to accommodate the combined action of fatigue and corrosion on flexible electrodes. Also, a previously custom-built wear tester was modified in order to accommodate tribo-corrosion testing of multi layered electrodes for photovoltaics.

- During testing, in-situ electrical resistance monitoring was performed in order to determine the crack onset strain or cycles to failure. Also, ex-situ surface characterization was performed in order to investigate the structural integrity of the electrodes after testing.

- FEA was used in order to predict the stress distribution and potential crack initiation on the electrodes. 
- DOE methods were used to investigate the effects and synergies of corrosion, applied strain, film thickness, and number of bending cycles and/or time on the electrical and structural integrity of the electrodes. Also, an empirical model was built to predict the increase in electrical resistance change based on statistical significant factors. Finally, a life-stress analysis was performed using the stresses obtained from FEA and cycles to failure values from mechanochemical experiments.

\subsection{Conclusions from the corrosion studies}

The corrosion behavior of ITO film on PET substrate was investigated in $0.05 \mathrm{M}$ acrylic acid to understand its stability in contact with acrylic adhesives. The steady state open circuit potential of ITO film is shifted to negative values with immersion time in $0.05 \mathrm{M}$ acrylic acid. This observation suggests an increase in the dissolution of ITO film in $0.05 \mathrm{M}$ acrylic acid with increasing immersion time. The potentiodynamic polarization results indicate an increase in $\mathrm{E}_{\text {corr }}$ and a decrease in $\mathrm{I}_{\text {corr }}$ to lower values with time. This suggests that the electro-activity of ITO film decreased with increasing immersion time. SEM investigations show corrosion of the ITO film whereas XPS analysis indicates a removal of indium from the ITO layer. The EIS measurements reveal two time constants and an increase in the charge transfer resistance of the Nyquist plot which suggests a decrease in the conductivity of ITO film with increasing immersion time. Both the electrochemical and surface characterization studies suggest a degradation of the conductivity of ITO film with increasing immersion time in $0.05 \mathrm{M}$ acrylic acid. 


\subsection{Conclusions from the mechano-chemical investigations}

The effects and synergies of acrylic acid environment, mechanical strain, film thickness, and NOC and/or time on the electrical and structural integrity of ITO and CNT films both deposited on PET substrates were investigated. CNT-based electrodes are observed to outperform their ITO counterparts. In the CNT case it is observed that fatigue leads to an increased CNT bundle exposed area, which in turn leads to increased electrode conductivity when in contact with acid medium.

It was also shown that the change in electrical resistance of ITO specimens under fatiguecorrosion is significantly higher than when they are subjected to corrosion, bending, bendingcorrosion, and fatigue. The severe cohesive and adhesive ITO delamination, from the base substrate observed during fatigue-corrosion is associated with the dramatic electrical resistance increase. This underlines the importance of the combined action of corrosion and fatigue. DOE analysis suggests that the effects of all designed factors are statistically significant. Change in electrical resistance is found to be higher at higher strains, higher thickness, and higher number of cycles when a corrosive environment is present. From FEA results, it is found that the midsection area across the width of the specimen is the most prone location for crack initiation. Finally, a life-stress relationship is established using the IPL-Weibull model to predict the life of such electrodes under the combined action of fatigue and corrosion.

\subsection{Conclusions from controlled-buckling studies}


Controlled buckling experiments under both tensile and compressive mode with in-situ monitoring of electrical resistance and optical microscopy were conducted in order to study the effects of shape and size on the mechanical properties of patterned ITO on PET substrates for flexible optoelectronics. Higher crack onset values were observed for the smaller size patterns as opposed to the larger size patterns. In addition, square-shaped patterns exhibited lower crack onset values. SEM results indicate cracking and crack-driven delamination under tensile and compressive buckling condition, respectively. Crack onset strain values based on microscopy do not correlate with crack onset strain values calculated based on an increase in electrical resistance and exhibit larger deviations when the patterns are placed under tension. In both cases the effect of the edge definition was highlighted.

\subsection{Conclusions from tribo-corrosion experimental work}

The tribology of model systems consisting of Ag, Ag-Pd, and Ag-Au alloy based films sputtered between two ITO layers was studied in a corrosive environment. Reciprocating PTFE ball-on-multilayer film tests were performed in a $\mathrm{NaCl}$ solution under a moderate contact pressure and an increasing number of testing cycles. Coating failure initiation, monitored by insitu electrical resistance measurements, occurs at a range between 200-268 reciprocating cycles. Three distinctive wear regimes are suggested according to weight measurements of the samples before and after testing. In the first regime, up to 540 cycles, no significant weight change is observed. A rapid wear regime follows up to 1080 reciprocating cycles. Above 1080 cycles, a third regime is observed at which a plateau phase is reached. Above 3240 cycles rapid weight loss of the PTFE ball is observed leading to material transfer and impregnation on the polymer 
surface as observed by XPS. EIS studies show that as the number of reciprocating sliding cycles increases the corrosion resistance of the structure decreases. Microscopic and profilometry results show microcutting and brittle ITO failure at low reciprocating cycles. As the number of cycles increases, the film is mostly removed. Also, base polymer substrate ploughing and plastic deformation are observed as well as PTFE material impregnation. PTFE wear mechanisms include abrasion, plastically deformed areas, and material transfer to the PET substrate.

In summary, the objectives of this work, investigating the electrochemical, electromechanical, and mechanochemical degradation behavior of transparent electrodes, have been met.

- During this study it was found that CNT-based components outperform their ITO counterparts when they are subjected to combined fatigue corrosion experiments. Also, for ITO electrodes under combined fatigue corrosion, externally applied strain was found to be the most critical factor for degradation. These findings underline the significance of the combined action of fatigue and corrosion.

- Patterned layer structures subjected to controlled-buckling experiments are observed to exhibit comparable crack onset strains as of their uniform layer counterparts. Results suggest that improving the pattern edge definition will be critical for improving the mechanical reliability of such structures.

- The life-stress relationship established using stress values obtained from FEA and cycles to failure data from mechanochemical experiments is important in the design of flexible electrodes. 


\section{RECOMMENDATIONS FOR FUTURE WORK}

\subsection{Mechanochemical studies of alternative electrodes}

One significant continuation of this study can be mechanochemical investigation of alternative materials such as multilayered structures (ITO-metal-ITO), graphene, and conductive polymers. The effects and synergies of various factors such as film thickness, acid concentrations, applied strain, number of cycles can be evaluated using design of experiment methods.

\subsection{Life-stress studies}

Another potential area can be comprehensive life-stress studies on different alternative materials using accelerated life test. Different life-stress models can be developed to predict the reliability of such materials. Developed models can help to establish a relationship between model parameters and electrode geometry such as width, thickness, and length.

\subsection{Ink-jet patterning of shapes}

In this project various shapes are patterned using photolithography. Results suggest that improving the pattern edge definition will be critical for improving the mechanical reliability of such structures. Therefore, in order to better understand edge effects, ink-jet printing of patterns 
at low temperature on polymer substrates can be employed. Also, less material can be used by avoiding photolithography patterning process.

\subsection{Insertion of Ag ink in ITO film crack openings}

In flexible optoelectronic device applications involving a stiff transparent conducting oxide layer such as ITO on a compliant substrate, cracking of the top layer is the main failure mechanism and a challenge for the development of truly flexible devices. This is due to the mismatch in mechanical and thermal properties exist in the hybrid system. As discussed in the previous chapters the ITO layer may lose its mechanical integrity and operational electrical conductivity due to cracking when the optoelectronic device conformed to various shapes during manufacturing and operation. In addition to looking for alternative durable transparent electrodes such as CNT, conductive polymers, and more recently graphene, there are methods which may contribute to the enhancement of the structural and electromechanical stability of conductive transparent oxide materials on compliant substrates. A multiple layer structure using highly stretchable and conductive metal such as Ag inserted between ITO layers has been suggested by many researchers previously [134-137] and investigated in this project under tribo-corrosion experiments. However, this layered structure exhibits reduced optical transmission. Another path which may lead to an enhancement of the electromechanical behaviors of the oxide layer may be the inclusion of metallic interconnects between the coating's crack openings. The innovation lies in putting the highly conductive Ag ink in the crack openings of ITO under different uniaxial applied strains. The following method can be followed in order to achieve this goal (Fig. 9.1). 
- ITO films sputtered on polymer substrates such as PEN will be strained uniaxially to generate cracks.

- Ag ink will be formulated by the reduction of silver nitrate $\left(\mathrm{AgNO}_{3}\right)$ to yield silver octanoate $\left(\mathrm{AgC}_{8} \mathrm{H}_{15} \mathrm{O}_{2}\right)$ precipitate which is then will be combined with xylene $\left(\mathrm{C}_{8} \mathrm{H}_{10}\right)$ solvent.

- Insertion on crack openings will be conducted using a small gauge needle tip luer-lock syringe.

- Curing of film will be performed by means of radiation-conduction-convection heating at temperatures as low as $150^{\circ} \mathrm{C}$.

- Optoelectronic and electromechanical characterization will be conducted in order to investigate the reliability of the films.
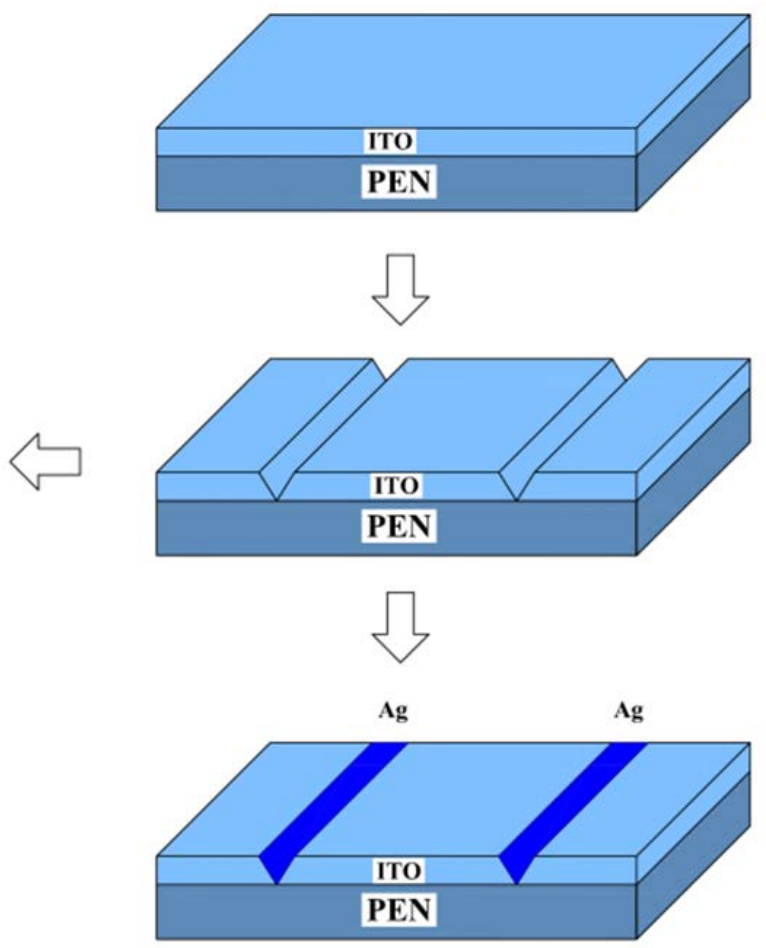

Fig. 9.1 Schematic of insertion of Ag ink in ITO crack openings. 
Such structures may be able to accommodate higher mechanical strains than their brittle layer counterparts during manufacturing and/or in service conditions. In addition, low electrical resistivity can be achieved due to the inclusion of the metallic interconnects. 


\section{REFERENCES}

[1] W.S. Wong, A. Salleo, In: W.S. Wong, A. Salleo (ed.) Flexible Electronics: Materials and Applications, Springer, 2009, p. v.

[2] M. Pagliaro, R. Ciriminna, G. Palmisano, ChemSusChem 1 (2008) 880.

[3] F. So, J. Kido, P. Burrows, MRS Bulletin 33 (2008) 663.

[4] K.A. Sierros, D.S Hecht, D.A. Banerjee, N.J. Morris, L. Hu, G.C. Irvin, R.S. Lee, D.R. Cairns, Thin Solid Films 518 (2010) 6977.

[5] T. Yamamura, M. Kitamura, K. Kuribayahi, Y. Arakawa, S. Takeuchi, MEMS 20 (2007) 739.

[6] J.H. Koo, J. Seo, T. Lee, Thin Solid Films, 524 (2012) 1.

[7] G.P. Crawford, In: G.P. Crawford (ed.) Flexible flat panel displays, John Wiley \& Sons, 2005, p. 1.

[8] K. Ellmer, Nature Photonics 6 (2012) 809.

[9] D.R. Cairns, In: G.P. Crawford (ed.) Flexible flat panel displays, John Wiley \& Sons, 2005, p. 163.

[10] D.R. Cairns, G.P. Crawford, Proceedings of the IEEE 93 (2005) 1451.

[11] Y.C. Lin, W.Q. Shi, Z.Z. Chen, Thin Solid Films 517 (2009) 1701.

[12] C. Guillen, J. Herrero, Thin Solid Films 520 (2011) 1.

[13] P. Mach, S.J. Rodriguez, R. Nortrup, P. Wiltzius, J.A. Rogers, Applied Physics Letters 78 (2011) 3592.

[14] J.H. Lee, D.N. Liu, S.T Wu, Introduction to flat panel displays, John Wiley \& Sons 2008, p. 1. 
[15] E. Hoggan, S.A. Brewster, J. Johnston, CHI Proceedings (2008) 1573-1582.

[16] D.R. Cairns, D.C. Paine, G.P. Crawford, Mat. Res. Soc. Symp. Proc. 666 (2001) 1.

[17] K.A. Sierros, D.A. Banerjee, D.R. Cairns, R. Bozich, SID Symposium Digest of Technical Papers 41 (2010) 882.

[18] C. J. Humphreys, MRS BULLETIN 33 (2008) 459.

[19] C. Bailey, C. Yin, H. Lu, C. Cartwright, EuroSimE (2011) 1.

[20] I.L. Azevedo, M.G. Morgan, F. Morgan, Proceedings of the IEEE 97 (2009) 481.

[21] M.R. Krames, O.B. Shchekin, R.M. Mach, G.O. Mueller, L Zhou, G. Harbers, M.G. Craford, Journal of Display Technology 3 (2007) 160.

[22] A. Zukauskas, M. Shur, R. Gaska, J. Wiley (2002) 133.

[23] K.H. Choi, J.A. Jeong, J.W. Kang, D.G. Kim, J.K. Kim, S.I. Na, D.Y. Kim, S.S. Kim, H.K. Kim, Solar Energy Materials \& Solar Cells 93 (2009) 1248.

[24] Y.S. Park, K.H. Choi, H.K. Kim, J. Phys. D: Appl. Phys. 42 (2009) 1.

[25] C.G. Granqvist, Solar Energy Materials \& Solar Cells 91 (2007) 1529.

[26] C. Lungenschmied, G. Dennler, H. Neugebauer, S.N. Sariciftci, M. Glatthaar, T. Meyer, A. Meyer, Solar Energy Materials \& Solar Cells 91 (2007) 379.

[27] S. Calnan, A.N. Tiwari, Thin Solid Films 518 (2010) 1839.

[28] K. Allen, In: G.P. Crawford (ed.) Flexible flat panel displays, John Wiley \& Sons, 2005, p. 495.

[29] M.C. Choi, Y. Kim, C.S. Ha, Prog. Polym. Sci. 33 (2008) 581.

[30] Y.Z. You, Y.S. Kim, D.H. Choi, H.S. Jang, J.H. Lee, D. Kim, Materials Chemistry and Physics 107 (2008) 444.

[31] D.H. Kim, M.R. Park, H.J. Lee, G.H. Lee, Applied Surface Science 253 (2006) 409. 
[32] W.A. MacDonald, M.K. Looney, D. MacKerron, R. Eveson, R. Adam, K. Hashimoto, K. Rakos, Journal of the SID 15/12 (2007) 1015.

[33] S. Logothetidis, Rev.Adv.Mater.Sci. 10 (2005) 387.

[34] B.A. MacDonald, K. Rollins, D. MacKerron, K. Rakos, R. Eveson, K. Hashimoto, B. Rustin, In: G.P. Crawford (ed.) Flexible Flat Panel Displays, John Wiley \& Sons, 2005, p. 11.

[35] T. Ma, B. Bhushan, Journal of Applied Polymer 88 (2003) 2082.

[36] E. Schwartz, Cornell University MSE 542(2006) 1.

[37] D.S. Ginley, J.D. Perkins, In: D.S. Ginley (ed.) Handbook of Transparent Conductors, Springer Science, 2010, p. 1.

[38] P.P. Edwards, A. Porch, M.O. Jones, D.V. Morgan, R.M. Perks, Dalton Transactions 19 (2004) 2995.

[39] B.Y. Oh, M.C. Jeong, T.H. Moon, W. Lee, J.M. Myoung, Journal of Applied Physics 99 (2006) 1.

[40] J.Y. Tseng, Y.T. Chen, M.Y. Yang, C.Y. Wang, P.C. Li, W.C. Yu, Y.F. Hsu, S.F. Wang, Thin Solid Films 517 (2009) 6310.

[41] J.I. Song, J.S. Park, H. Kim, Y.W. Heo, J.H. Lee, J.J. Kim, G.M. Kim, B.D. Choi, Applied Physics Letters 90 (2007) 1.

[42] K. Ellmer, J. Phys. D: Appl. Phys. 34 (2001) 3097.

[43] Z.L. Pei, X.B. Zhang, G.P. Zhang, J. Gong, C. Sun, R.F. Huang, L.S. Wen, Thin Solid Films 497 (2006) 20.

[44] T.L. Chen, D.S. Ghosh, D. krautz, S. Cheylan, V. Pruneri, Applied Physics Letters 99 (2011) 1.

[45] M.H. Andrew Ng, L.T. Hartadi, H. Tan, C.H. Patrick Poa, Nanotechnology 19 (2008) 1. 
[46] U. Lang, N. Naujoks, J. Dual, Synthetic Metals 159 (2009) 473.

[47] Y. Xia, K. Sun, J. Ouyang, Advanced Materials 24 (2012) 2436.

[48] J. Lewis, S. Grego, B. Chalamala, E. Vick, D. Temple, Applied Physics Letters 85 (2004) 16.

[49] C. Coutal, A. Azema, J.C. Roustan, Thin Solid Films 288 (1996) 248.

[50] D.C. Paine, H.Y. Yeom, B. Yaglioglu, In: G.P. Crawford (ed.) Flexible Flat Panel Displays, John Wiley \& Sons, 2005, p. 79.

[51] H.S. Kwok, X.W. Sun, D.H. Kim, Thin Solid Films 335 (1998) 299.

[52] S.O. Yoon, H.J. Jung, K.H. Yoon, Solid State Communications 64 (1987) 617.

[53] H. Ma, J.S. Cho, C.H. Park, Surface and Coatings Technology 153 (2002) 131.

[54] W.F. Wu, B.S. Chiou, Thin Solid Films 293 (1997) 244.

[55] Q. Cao, J.A. Rogers, ACS Nano 2 (2008) 1266.

[56] J.L. Blackbum, T.M. Barnes, M.C. Beard, Y.H. Kim, R.C. Tenent, T.J. McDonald, B. To, T.J. Coutts, M.J. Heben, Adv. Mater. 21 (2009) 29.

[57] E.M. Doherty, S. De, P.E. Lyons, A. Shmeliov, P.N. Nirmalraj, V. Scardaci, J. Joimel, W.J. Blau, J.J. Boland, J.N. Coleman, Carbon 47 (2009) 2466.

[58] Y.F. Lan, W.C. Peng, Y.H. Lo, J.L. He, Materials Research Bulletin 44 (2009) 1760.

[59] M.G. Sandoval-Paz, R. Ramirez-Bon, Thin Solid Films 517 (2009) 2596.

[60] L.M. Wang, Y.J. Chen, J. W. Liao, Journal of Physics and Chemistry of Solids 69 (2008) 527.

[61] T.K. Yong, T.Y. Tou, B.S. Teo, Applied Surface Science 248 (2005) 388.

[62] G. Gruner, Journal of Materials Chemistry 16 (2006) 3533. 
[63] C.M. Aguirre, S. Auvray, S. Pigeon, R. Izquierdo, P. Desjardins, R. Martel, Applied Physics Letters 88 (2006) 183104.

[64] K. Maki, N. Komiya, A. Suzuki, Thin Solid Films 445 (2003) 224.

[65] J. Lee, H. Jung, J. Lee, D. Lim, K. Yang, J. Yi, W.C. Song, Thin Solid Films 516 (2008) 1634.

[66] D.P. Norton, In: R. Eason (ed.) Pulsed Laser Deposition of Thin Films: Applications-Led Growth of Functional Materials, John Wiley \& Sons, 2007, p. 3.

[67] J.A. Thornton, J.E. Greene, In: R.F. Bunshah (ed.) Handbook of Deposition Technologies for Films and Coatings: Science, Technology and Applications, Noyes Publications, 1994, p. 275.

[68] K. Wasa, M. Kitabatake, H. Adachi, Thin Film Materials Technology: Sputtering of Compound Materials, William Andrew, 2004, p. 115.

[69] P.C.P. Bouten, P.J. Slikkerveer, Y. Leterrier, In: G.P. Crawford (ed.) Flexible Flat Panel Displays, John Wiley \& Sons, 2005, p. 99.

[70] Y. Leterrier, L. Boogh, J. Andersons, J.A.E. Manson, Journal of Polymer Science Part B: Polymer Physics 35 (1997) 1449.

[71] D.R. Cairns, R.P. Witte II, D.K. Sparacin, S.M. Sachsman, D.C. Paine, G.P. Crawford, Applied Physics Letters 76 (2000) 1425.

[72] Y. Leterrier, L. Medico, F. Demarco, J.A.E. Manson, U. Betz, M.F. Escola, M.K. Olsson, F. Atamny, Thin Solid Films 460 (2004) 156.

[73] M.M. Hamasha, K. Alzoubi, S. Lu, Journal of Display Technology 7 (2011) 426.

[74] Z. Chen, B. Cotterell, W. Wang, Engineering Fracture Mechanics 69 (2002) 597.

[75] Z. Chen, B. Cotterell, W. Wang, E. Guenther, S.J. Chua, Thin Solid Films 394 (2001) 202. 
[76] A.A. Abdallah, P.C.P. Bouten, G. de With, Engineering Fracture Mechanics 77 (2010) 2896.

[77] A.A. Abdallah, P.C.P. Bouten, J.M.J. den Toonder, G. de With, Surface \& Coatings Technology 205 (2011) 3103.

[78] C.W. Yang, J.W. Park, Surface \& Coatings Technology 204 (2010) 2761.

[79] C.M. Trottier, P. Glatkowski, P. Wallis, J. Luo, Journal of the SID 13/9 (2005) 759.

[80] S.P. Gorkhali, D.R. Cairns, G.P. Crawford, Journal of the SID 12 (2004) 45.

[81] K. Alzoubi, M.M. Hamasha, S. Lu, B. Sammakia, Journal of Display Technology 7 (2011) 593.

[82] T. Koniger, H. Munstedt, Meas. Sci. Technol. 19 (2008) 1.

[83] S.H. Choa, C.K. Cho, W.J. Hwang, K.T. Eun, H.K. Kim, Solar Energy Materials \& Solar Cells 95 (2011) 3442.

[84] Y.F. Lan, W.C. Peng, Y.H. Lo, J.L. He, Organic Electronics 11 (2010) 670.

[85] Y. Leterrier, D. Pellaton, D. Mendels, R. Glauser, J. Andersons, J.A.E. Manson, Journal of Materials Science 36 (2001) 2213.

[86] J. Andersons, Y. Leterrier, I. Fescenko, Thin Solid Films 434 (2003) 203.

[87] Y.N. Kim, M.S. Jeon, M.C. Shin, S.M. Lee, H.S. Lee, Key Engineering Material 317-318 (2006) 381.

[88] K.A. Sierros, N.J. Morris, S.N. Kukureka, D.R. Cairns, Wear 267 (2009) 625.

[89] A.A. Tseng, Applied Surface Science 256 (2010) 4246.

[90] K.A. Sierros, A.J. Kessman, R. Nair, N.X. Randall, D.R. Cairns, Thin Solid Films 520 (2011) 424. 
[91] D.S. Hecht, D. Thomas, L. Hu, C. Ladous, T. Lam, Y. Park, G. Irvin, P. Drzaic, Journal of the SID 17/11 (2009) 941.

[92] P.K. Pandey, A. Srivastava, J. Tripathy, K. Behari, Carbohydrate Polymers 65 (2006) 414.

[93] G. Folcher, H. Cachet, M. Froment, J. Bruneaux, Thin Solid Films 301 (1997) 242.

[94] P.M.S. Monk, C.M. Man, Journal of Materials Science: Materials in Electronics 10 (1999) 101.

[95] R.H. Jones, R.E. Ricker, In: R.H. Jones (ed.) Stress-Corrosion Cracking, ASM International, 1992, p. 1.

[96] K. Ramji, D.R. Cairns, K.A. Sierros, S.N. Kukureka, SID Symposium Digest of Technical Papers 38 (2007) 1790.

[97] K.A. Sierros, N.J. Morris, K. Ramji, D.R. Cairns, Thin Solid Films 517 (2009) 2590.

[98] M.M. Hamasha, K. Alzoubi, S. Lu, S.B. Desu, Thin Solid Films 519 (2011) 6033.

[99] T. Martin, A. Christou, IEEE International Reliability Physics Symposium 47 (2009) 117.

[100] C.Y. Peng, M.M. Hamasha, D. VanHart, S. Lu, C.R. Westgate, IEEE Transactions on Device and Materials Reliability 13 (2013) 236.

[101] D.G. Neerinck, T.J. Vink, Thin Solid Films 278 (1996) 12.

[102] W.A. MacDonald, Journal of Materials Chemistry 14 (2004) 4.

[103] H.H. Hassan, E. Abdelghani, M.A. Amin, Electrochimica Acta 52 (2007) 6359.

[104] P.K. Biswas, A. De, L.K. Dua, L. Chkoda, Applied Surface Science 253 (2006) 1953.

[105] K.L. Purvis, G. Lu, J. Schwartz, S.L. Bernasek, J. Am. Chem. Soc. 122 (2000) 1808.

[106] A. Bessiere, J.C. Badot, M.C. Certiat, J. Livage, V. Lucas, N. Baffier, Electrochimica Acta 46 (2001) 2251.

[107] C.A. Huang, K.C. Li, G.C. Tu, W.S. Wang, Electrochimica Acta 48 (2003) 3599. 
[108] E. Matveeva, Journal of Electrochemical Society 152 (2005) H138.

[109] C.M. Welch, O. Nekrassova, R.G. Compton, Talanta 65 (2005) 74.

[110] M.R. Pitts, J.R. Harrison, C.J. Moody, J. Chem. Soc., Perkin Trans. 1 (2001) 955.

[111] M. Senthilkumar, J. Mathiyarasu, J. Joseph, K.L.N. Phani, V. Yegnaraman, Materials Chemistry and Physics 108 (2008) 403.

[112] A. Kraft, H. Hennig, A. Herbst, K.H. Heckner, Journal of Electroanalytical Chemistry 365 (1994) 191.

[113] W.S. Leung, Y.C. Chan, S.M. Lui, Microwave Conference (2008) 1.

[114] F. Zhu, C.H.A. Huan, K. Zhang, A.T.S. Wee, Thin Solid Films 359 (2000) 244-250.

[115] M. Serantoni, V.J. Cunnane, Journal of Electroanalytical Chemistry 548 (2003) 49.

[116] H.J. Lin, D.Y. Lin, J.S. Wu, C.S. Yang, W.C. Chou, W.H. Lo, J.S. Wang, Japanese Journal of Applied Physics 48 (2009) 04C122.

[117] A. Benedetto, M. Balog, H. Rayah, F. L. Derf, P. Viel, S. Palacin, M. Sall, Electrochimica Acta 53 (2008) 3779.

[118] R.M. Souto, L.F. Merida, S. Gonzalez, D.J. Scantlebury, Corrosion Science 48 (2006) 1182.

[119] M. Mahdavian, M.M. Attar, Corrosion Science 48 (2006) 4152.

[120] N. Bonanos, B.C.H. Steele, E.P. Butler, In: J.R. Macdonald (ed.) Impedance spectroscopy, John Wiley\& Sons, 1987, p.261.

[121] I.A. Ryzhikov, A.A. Pukhov, A.S. Ilin, N.P. Glukhova, K.N. Afanasiev, A.S. Ryzhikov, Microelectronic Engineering 69 (2003) 270.

[122] C.T. Chu, P.D. Fuqua, J.D. Barrie, Applied Optics 45 (2006) 1583. 
[123] H.Z. Geng, K.K Kim, K.P. So, Y.S. Lee, Y. Chang, Y.H. Lee, J. Am. Chem. Soc. 129 (2007) 7758.

[124] P.N. Nirmalraj, P.E. Lyons, S. De, J.N. Coleman, J.J. Boland, Nano Letters 9 (2009) 3890.

[125] D.S. Hecht, K.A. Sierros, R.S. Lee, C. Ladous, C. Niu, D.A. Banerjee, D.R. Cairns, Journal of the SID 19/2 (2011) 157.

[126] J.C. Anderson, K.D. Leaver, R.D. Rawlings, J.M. Alexander, Material Science, Chapmand \& Hall, 1990, p. 181.

[127] K. Alzoubi, S. Lu, B. Sammakia, M. Poliks, Journal of Display Technology 7 (2011) 348.

[128] K. Rajkumar, K. Kundu, S. Aravindan, M.S. Kulkarni, Materials and Design 32 (2011) 3029.

[129] H.D. Espinosa, B.C. Prorok, Journal of Materials Science 38 (2003) 4125.

[130] G.A. Potoczny, T.S. Bejitual, J.S. Abell, K.A. Sierros, D.R. Cairns, S.N. Kukureka, Thin Solid Films 528 (2013) 205.

[131] J. C. C. Fan, F. J. Bachner, Appl. Optics 15 (1976) 1012.

[132] C. E. Kennedy, K. Terwilliger, Transactions of the ASME-N-Journal of Solar Engineering 127 (2005) 262.

[133] K. Chiba, T. Takahashi, T. Kageyama, H. Oda, Appl. Surf. Sci. 246 (2005) 48.

[134] M. Fahland, P. Karlsson, C. Charton, Thin Solid Films 392 (2001) 334.

[135] J. Lewis, S. Grego, B. Chalamala, E. Vick, D. Temple, Applied Physics Letters 85 (2004) 3450.

[136] K.H. Choi, H.J. Nam, J.A. Jeong, S.W. Cho, H.K. Kim, J.W. Kang, D.G. Kim, W.J. Cho, Applied Physics Letters 92 (2008) 223302.

[137] H.J. Park, J.H. Park, J.I. Choi, J.Y. Lee, J.H. Chae, D. Kim, Vacuum 83 (2009) 448. 